\title{
The biological role of Fusarium graminearum mycotoxins
}

\author{
Dissertation \\ to obtain the Ph. D. degree \\ in the International Ph. D. Program for Agricultural Sciences in Goettingen (IPAG) \\ at the Faculty of Agricultural Sciences, \\ Georg-August-University Göttingen, Germany
}

presented by

\section{Awais Ahmed}

born in Nawabshah, Pakistan.

Göttingen, September 2010 


\section{D7}

1. Name of supervisor: Prof. Dr. Petr Karlovsky

2. Name of co-supervisor: Prof. Dr. Elke Pawelzik

Date of dissertation: 18.11.2010 
'In the loving memory of (Ammi) Rehana and (Abbu) J abbar who believed in education and (Amma) Fatima who sacrificed everything for our family'. 


\section{Contents}

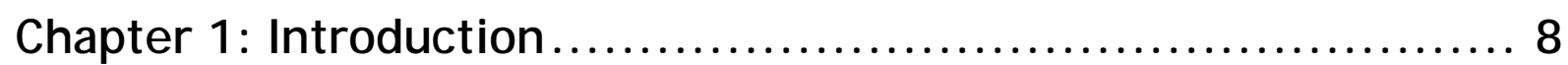

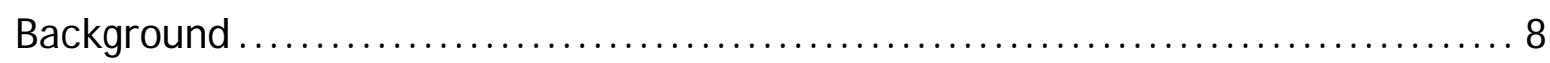

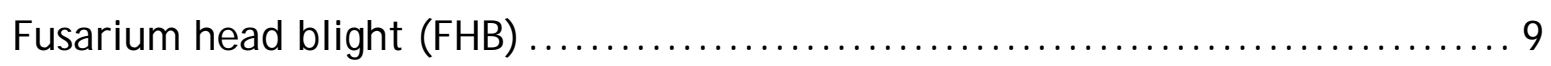

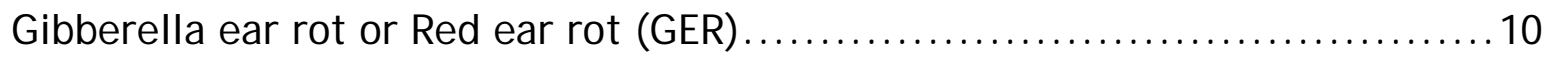

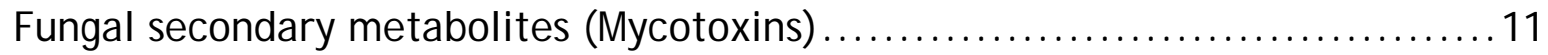

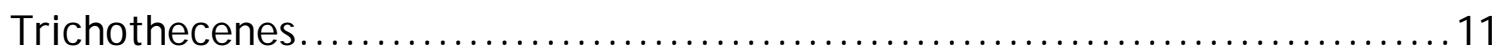

Broad spectrum effects of trichothecenes on animals ...................... 12

Broad spectrum effects of trichothecenes on plants...................... 12

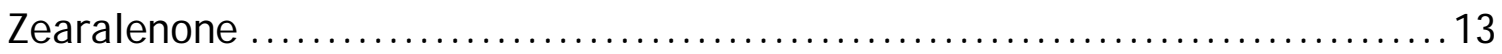

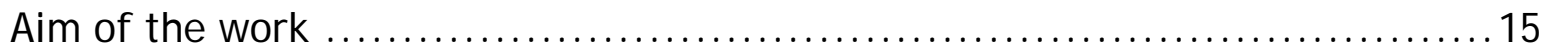

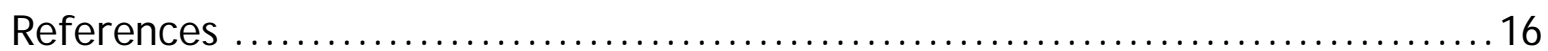

Chapter 2: Role of zearalenone in the interaction of F. graminearum with G. roseum in vitro and in wheat and maize rhizosphere ....... 23

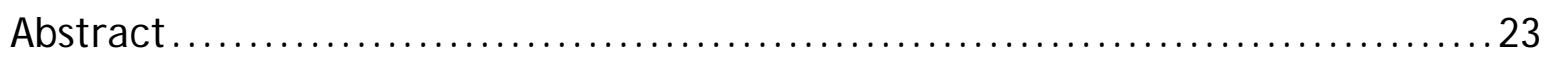

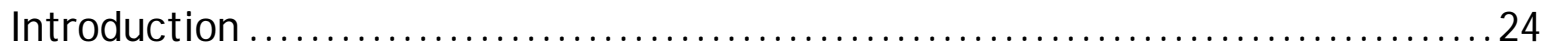

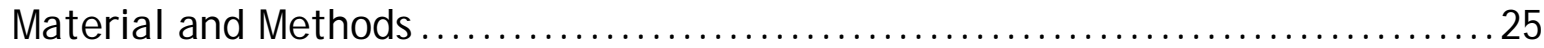

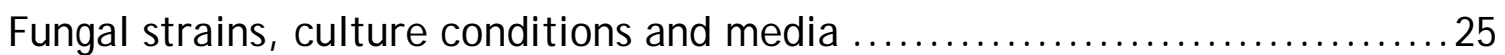

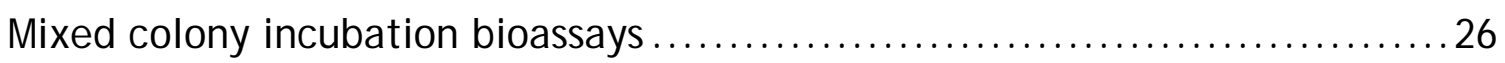

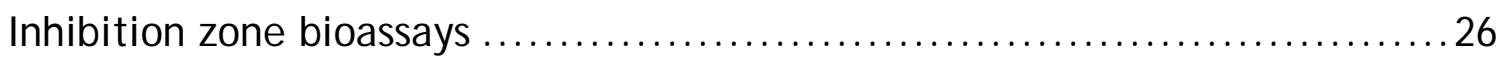

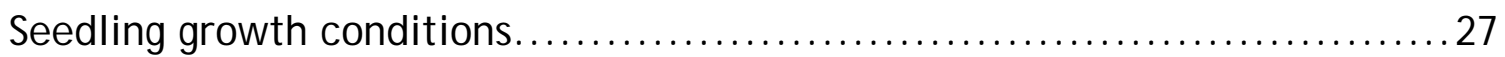

Aggressiveness test on maize and wheat root ............................ 27

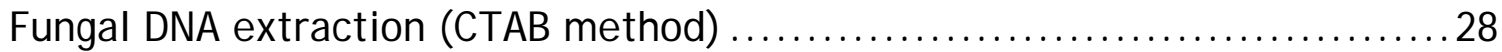

F. graminearum DNA quantification by Real- time PCR ................... 28

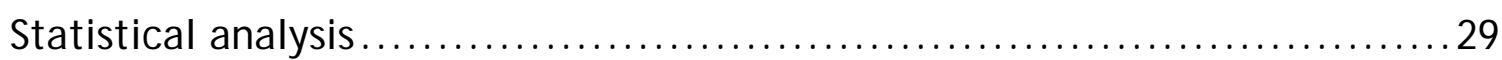

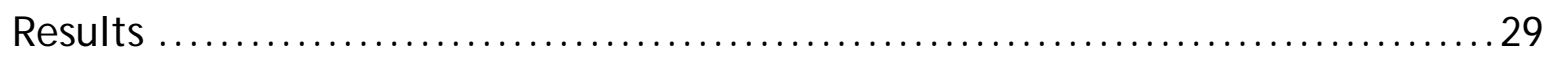

Mixed colony incubation bioassays .................................... 29

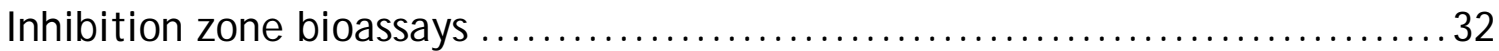

Ecological role of ZEN and maize seedlings protection with G. roseum ......... 33

Ecological role of ZEN and wheat seedlings protection with G. roseum ......... 38

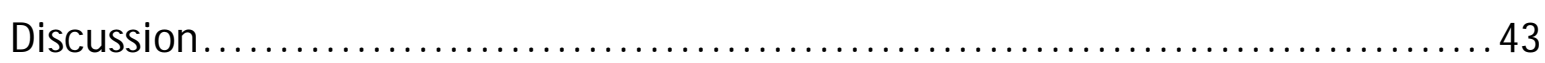

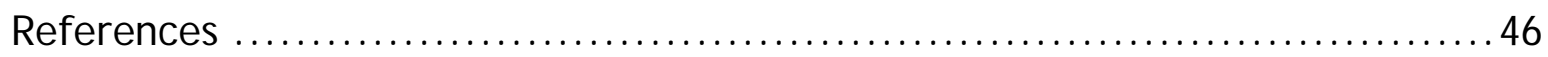

Chapter 3: Pathogenicity and mycotoxin production of different

F.graminearum strains on maize and wheat ear and root infection 49 


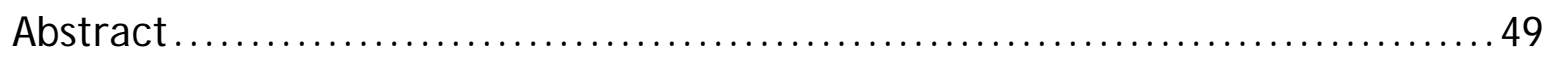

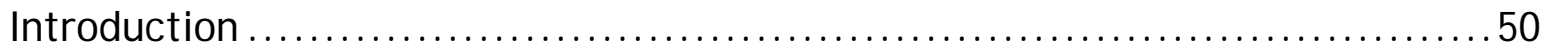

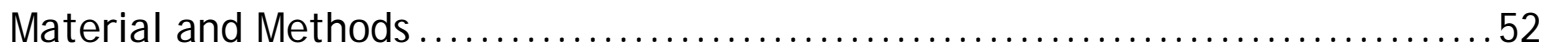

Fungal strains, culture conditions and media ............................ 52

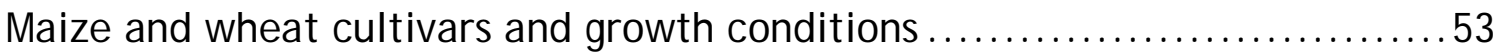

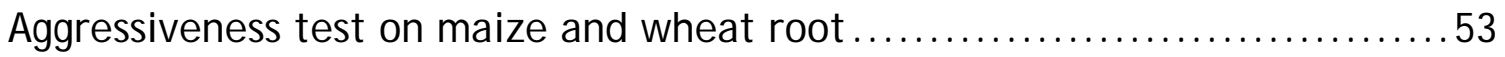

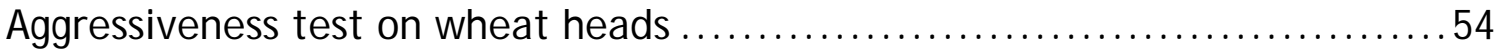

Maize ears trial design, infection method and disease rating.................. 55

DNA extraction of wheat and maize flour (CTAB method) ................... 55

F. graminearum DNA quantification by Real Time PCR $\ldots \ldots \ldots \ldots \ldots \ldots \ldots 56$

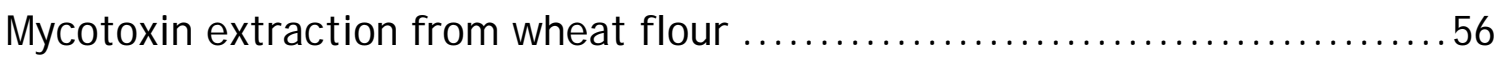

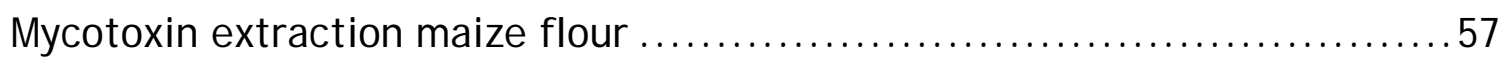

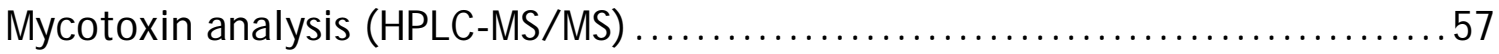

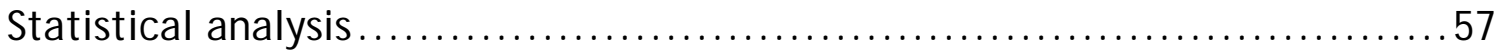

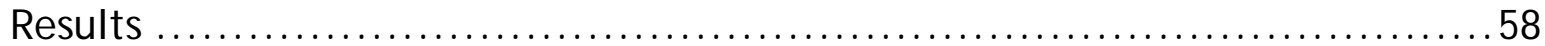

F. graminearum aggressiveness on maize and wheat root $\ldots \ldots \ldots \ldots \ldots \ldots \ldots 58$

F. graminearum aggressiveness on wheat heads.......................... 61

F. graminearum aggressiveness on maize ears .......................... 67

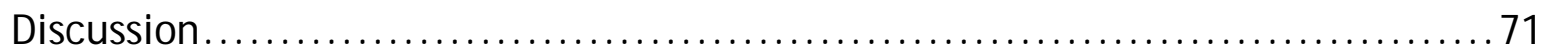

F. graminearum aggressiveness on maize and wheat root $\ldots \ldots \ldots \ldots \ldots \ldots \ldots 71$

F. graminearum aggressiveness on wheat heads......................... 72

F. graminearum aggressiveness on maize ears........................... 73

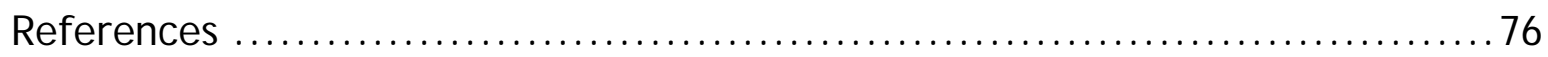

Chapter 4: Role of deoxynivalenol (DON) and nivalenol (NIV) in wheat and maize root infection by $F$, graminearum ............... 81

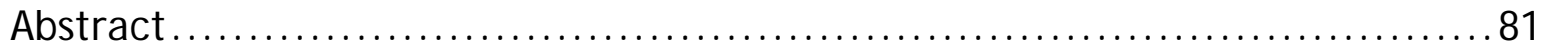

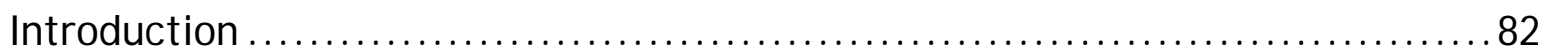

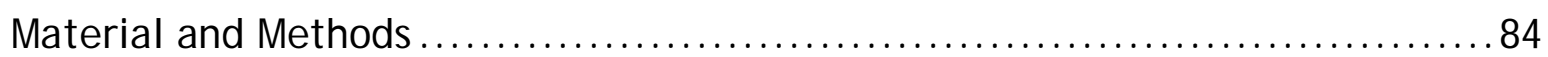

Fungal strains, culture conditions and media ........................... 84

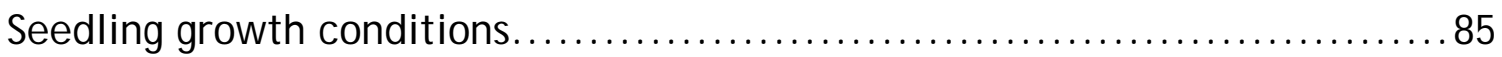

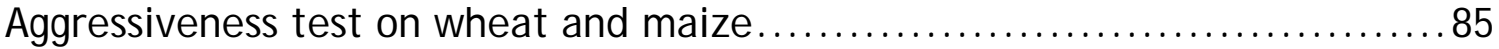

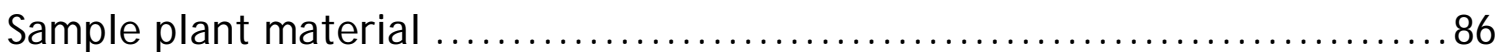

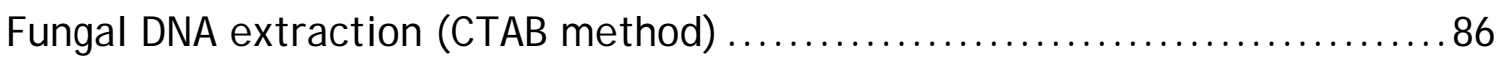

F. graminearum DNA quantification by Real Time PCR $\ldots \ldots \ldots \ldots \ldots \ldots \ldots . \ldots 6$

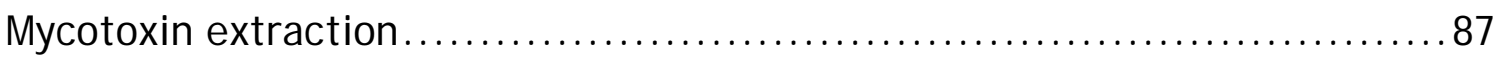




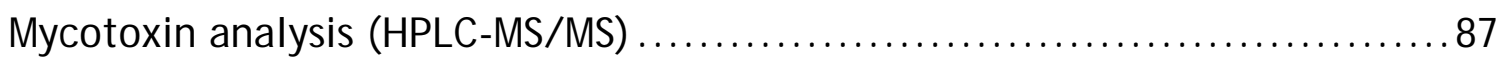

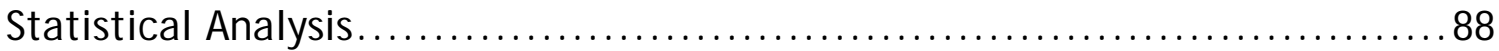

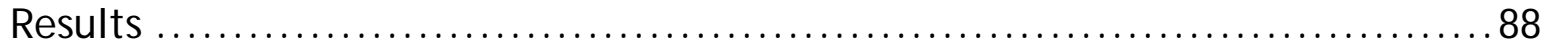

F. graminearum aggressiveness on wheat (cv. Apogee) root ................ 88

F. graminearum aggressiveness on maize (cv. Gaspe flint) root ............. 96

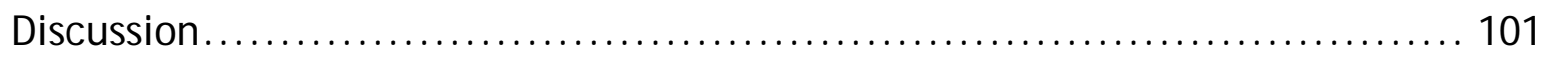

F. graminearum aggressiveness on wheat (cv. Apogee) root ............... 101

F. graminearum aggressiveness on maize (cv. Gaspe flint) root ............ 103

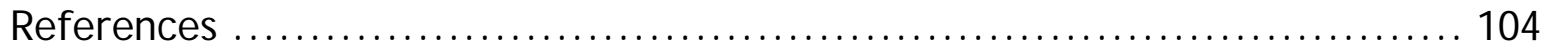

Chapter 5: General Discussion................................. 107

Role of zearalenone in the interaction of $F$. graminearum with $G$. roseum in vitro

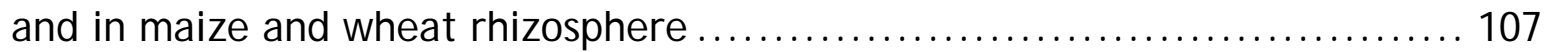

Pathogenicity and mycotoxin production of different F.graminearum strains on maize and wheat ear and root infection................................ 109

Role of deoxynivalenol (DON) and nivalenol (NIV) in wheat and maize root

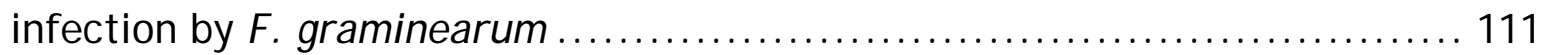

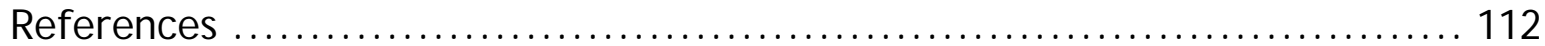

Summary $\ldots \ldots \ldots \ldots \ldots \ldots \ldots \ldots \ldots \ldots \ldots \ldots \ldots \ldots \ldots \ldots \ldots \ldots \ldots \ldots \ldots \ldots \ldots \ldots \ldots \ldots \ldots, 116$

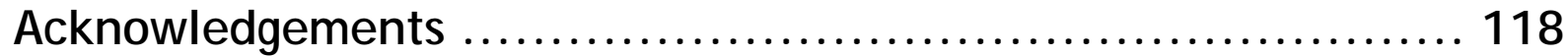




\section{Chapter 1: Introduction}

\section{Background}

F. graminearum Schwabe (teleomorph Gibberella zeae [Schwein] Petch) is one of the most toxigenic species in the genus Fusarium. The fungus is adapted to different ecological niches world wide, both as a saprophyte and as a pathogen under diverse environmental conditions (Parry et al., 1995). F. graminearum is a devastating pathogen of small-grain cereals, causing seedling blight and root rot as well as head blight (FHB) in wheat and ear rot (FER) in maize (Goswami and Kistler, 2004; Munkvold, 2003; Sutton, 1982). Fusarium infections in wheat, barley and maize cause potential yield losses world-wide due to inferior grain quality and are therefore of great economic significance (McMullen et al., 1997; Parry et al., 1995; Sutton, 1982). Being as a notorious pathogen, it is considered to be the most prolific producer of trichothecenes type $B$ and the estrogenic mycotoxin zearalenone. The trichothecenes deoxynivalenol (DON) and its acetylated derivatives and nivalenol (NIV) are common contaminants in cereals and maize. These cause a potential health risk when contaminated grain is used for in human and animal food production (D'Mello and Macdonald, 1997; D'Mello et al., 1999). Zearalenone (ZEN) has estrogenic activity and can be a significant contaminant of maize, barley, wheat and other cereals (Hagler et al., 2001; Vidnes et al., 2003). The ZEN contaminated food or feed causes hyperestrogenism, especially in pigs, and reproductive problems in experimental animals and livestock (Desjardins and Proctor, 2001). Directives from the European Union set the maximum limit for ZEN in unprocessed cereals other than corn to $100 \mathrm{ppb}$ and unprocessed maize to 200 ppb. For DON, the maximum limit in unprocessed cereals other than durum wheat, oats and corn is set to $1250 \mathrm{ppb}$ and for unprocessed maize to $1750 \mathrm{ppb}$ (The commission of the European communities., 2006). The infection or colonization of cereal grains or maize kernels not only affects yield and leads to contamination with zearalenone, but can also result in contamination of grains with trichothecenes. Most of the known trichothecenes are toxic to cells, and the broad spectrum effects on eukaryotic organisms, is a manifestation of their effects at the cellular level. The effects of these toxins on eukaryotic cells have been extensively 
investigated in animals but are not yet fully understood in plants (Rocha et al., 2005).

There have been enormous efforts done to elucidate the role of these mycotoxins in pathogenicity and host-specific-interactions on different crops (Bai et al., 2002; Desjardins and Hohn, 1997; Harris et al., 1999; Maier et al., 2006). The economic and health implications are accessed so far to encompass the two most common diseases displayed by F. graminearum which is FHB in wheat and FER in maize.

\section{Fusarium head blight (FHB)}

Fusarium head blight (FHB) is the most devastating disease caused by F. graminearum. The fungus overwinters as saprophytic mycelia in crop debris, especially in maize residues. In the proceeding season, warm moist climatic condition and seasonal shift favors the development and maturation of conidia and perithecia that produce ascospores concurrently with the flowering of cereal crops (Goswami and Kistler, 2004). The sticky ascospores are forcibly discharged from mature perithecia formed on the surface of the crop debris (Trail et al., 2005) and dispersed by wind, rain or insects to the host plants (Parry et al., 1995; Sutton, 1982). The depositions of the spores take place on the spike tissue, where the infection process initiates; wheat heads are most susceptible to infection during anthesis (Bushnell et al., 2003; Sutton, 1982). The fungal hyphae develops on the exterior surfaces of florets and glumes that allow it to grow towards the stomata within the inflorescence (Bushnell et al., 2003; Goswami and Kistler, 2004). Sometimes hyphae structures are formed between cuticle and epidermal cell wall on the surface of glumes (Pritsch et al., 2000). This subcuticular growth on the glume, lemma and palea is thought to serve as a mechanism for fungal spread (Bushnell et al., 2003). Other entry routes include the stomata and underlying parenchyma, exposed anthers, openings between the lemma and palea of the spikelet during dehiscence (Bushnell et al., 2003; Lewandowski and Bushnell, 2001) and through the wheat glumes base, where the epidermis and parenchyma are thin-walled. Once the pathogen enters into the floret, the anthers and stigmas are most easily colonized. The pathogen spreads in wheat from floret to floret inside a spikelet and from spikelet to spikelet through the vascular bundles in the rachis and rachilla (Ribichich et al., 2000). During humid conditions, mycelia can spread 
on the exteriors of the glume, lemma and palea in both wheat and barley (Bushnell et al., 2003). The pathogen sometimes switches briefly between biotrophic and necrotrophic phases. The necrotrophic shift is associated with increased colonization and the eventual death of the plant accompanied by substrate depletion. In some cases, F. graminearum may also colonize plant tissues asymptomatically, such as stalks of corn (Bushnell et al., 2003).

\section{Gibberella ear rot or Red ear rot (GER)}

Fusarium Red ear rot or Gibberella ear rot (GER) is a major disease of maize caused by Fusarium graminearum. The fungus survives saprophytic on maize stubbles or other residues from the preceded crops (Smith and White, 1988). During the saprophytic phase, it produces chlamydospores on crop residues to survive periods between host crops, and macroconidia in sporodochia (Sutton, 1982). Maize residues are reported as the most significant source of $F$. graminearum inoculum causing (FHB) of wheat (Dill-Macky and J ones, 2000; Schaafsma et al., 2001). The dispersal of macroconidia or ascospores is initially trajected by water splashes and incorporated into the atmospheric population of $F$. graminearum propagules (Bergstrom and Shields, 2002), showing that once they are dispersed from the sporodochium, they can overcome relatively long distances of several kilometers. The primary infection pathway in maize kernels is via silk channel and susceptibility remains high during the first 6 days after silk emergence (Reid and Hamilton, 1996). The pathogen usually initiates from the tip of the ear and develops a red or pink mold covering a large proportion of the ear (Miller, 1994; Munkvold, 2003; Smith and White, 1988). Spores can reach the silks not only by water splash or wind dispersal, but also through insect vectors. Some infections to the kernels are clearly initiated by direct injury through birds or lepidopteran insects, but this appears to be a less important pathway than silk route. GER outbreaks are common in cooler areas or areas with higher precipitation during the growing season (Logrieco et al., 2002; Smith and White, 1988). 


\section{Fungal secondary metabolites (Mycotoxins)}

Mycotoxins are defined as low molecular weight fungal secondary metabolites that are toxic to vertebrates (Desjardins and Hohn, 1997). These secondary metabolites are a remarkably diverse class of cellular products that often exhibit taxonomic specificity. They are organic compounds which are produced by routes other than the normal metabolic pathways after a period of active growth, development or reproduction of organisms. Mycotoxins are not thought to be essential for the growth of the organism under culture conditions (Bennett, 1983; Bennett, 1995; Bode et al., 2002). The biological function of mycotoxins remains a topic of great speculation. However, they are considered to play a significant role in fungal defence, substrate colonization, and interspecies competition for its producer in its ecological niche.

\section{Trichothecenes}

So far 150 trichothecenes and their derivatives have been isolated and characterized (Gutleb et al., 2002). These non-volatile, low-molecular- weight compounds (Wannemacher et al., 2000) are varied in chemical structure in both the position and the number of hydroxylations, as well as esterifications (Desj ardins et al., 1993). They are thermo stable and do not degrade in normal food processing (Eriksen, 2003). The trichothecenes type A and B are the most prevalent contaminants in cereals and maize along with the estrogenic mycotoxin ZEN (D'Mello and Macdonald, 1997; D'Mello et al., 1999). Therefore, the major products of the trichothecene biosynthetic pathway are known as T-2 toxin and diacetoxyscirpenol (DAS) as type A trichothecenes and deoxynivalenol (DON) and nivalenol (NIV) as type B trichothecenes (Ueno, 1985). One of the major producers of type $B$ trichothecenes is ( $F$. graminearum) which can be grouped into two chemotypes based on whether they produce DON (or acetylated derivatives) or NIV (Chandler et al., 2003; Lee et al., 2001). 


\section{Broad spectrum effects of trichothecenes on animals}

The effects of trichothecenes have been documented mainly on humans and animals rather than on plants (Pestka, 2008; Pestka and Smolinski, 2005; Richard, 2007; Rocha et al., 2005). The large-scale effects of selective trichothecenes on animals include growth retardation, reduced ovarian function and reproductive disorders, immunocompromization, feed refusal and vomiting (SCF ., 2002). The growth retardation has been reported in mice after DON and NIV intake (Ohtsubo et al., 1989; Ryu et al., 1988), increased postnatal mortality caused by DON (Khera et al., 1984) and intrauterine growth retardation caused by NIV (Ito et al., 1986). DON is also found to increase the susceptibility of mice to infections (Tryphonas et al., 1986). Moreover, lymphoid cells and fibroblasts were the most sensitive cell types to DON (Charoenpornsook et al., 1998; Reubel et al., 1987). A special feature of DON toxicity is the characteristic induction of vomiting (DON is also called vomitoxin) and feed refusal, as seen in pigs, or delayed gastric emptying and feed refusal, as observed in rats and mice (SCF ., 1999). The emetic effect is thought to be mediated through the serotonergic activity in the central nervous system (SCF ., 1999). Nevertheless, it may not always be possible to anticipate from animal to plant systems because of differences in their trichothecenes uptake or metabolism.

\section{Broad spectrum effects of trichothecenes on plants}

The large-scale phytotoxic effects were also recently reviewed by (Rocha et al., 2005). The general trichothecene effects are evaluated in some axenic and in vitro culture studies. Shimada and Otani (1990) reported that NIV was 10-24-fold less toxic than DON to seedlings of seven wheat cultivars. Eudes et al. (2000) demonstrated the phytotoxic effects of the six trichothecenes DON, 3acetyldesoxynivalenol (3ADON), NIV, DAS, T-2 toxin and HT-2 in four wheat cultivars. They found that DON and 3ADON were more toxic than T-2 toxin, HT-2 and DAS, and that these toxins inhibited wheat coleoptile elongation even at very low concentrations. Wakulinski (1989) used seedlings of three varieties of winter wheat "Grana", "Emika", and "SMH-68-4" to evaluate the phytotoxicity of six Fusarium metabolites. He observed that T-2 toxin was less phytotoxic than DON, but more toxic than DAS and reported that DON and 3-ADON were the most potent 
inhibitors of germination and of root and leaf mass increase. (Bruins et al., 1993; McLean, 1996) summarized the phytotoxic effects of DON on plants as growth retardation and inhibition of seedling and green plant regeneration. Bruins et al. showed that DON inhibited the growth of wheat seedlings, coleoptile segments, anther-derived callus, anther-derived embryos and green plant regeneration. DON also inhibited wheat root growth during germination (Shimada and Otani, 1990). Moreover, Wang and Miller (1998) observed that DON strongly inhibited coleoptile growth of wheat cultivars with different levels of resistance to FHB, however they found cultivars resistant to FHB were more tolerant to DON and 3-ADON than those that were susceptible. The gene-knock-out studies have further revealed the potential role of DON and NIV in fungal virulence. Wang et al. (2006) reported that F. graminearum DON (Tri5-) disrupted mutants were less aggressive on wheat, barley and triticale seedling root infection compared to wild-type (Tri5+). Moreover, Bai et al. (2002) inoculated the wheat heads with F. graminearum DON (Tri5-) deficient strains; the fungus was able to produce the initial infection but was unable to spread beyond the whole spike unlike the wild-type (Tri5t), Maier et al. (2006) inoculated the maize cobs with F. graminearum NIV (Tri5-) disrupted strains and found out that the greater virulence was caused only by NIV wild-type (Tri5+) compared to a NIV deficient mutants (Tri5-). In another study Harris et al. (1999) tested F. graminearum DON (Tri5-) in field trials of maize and reported that the deficient mutants were not as virulent as their wild-type counterparts. All these studies help to elucidate the role of DON and NIV as a virulence factor for fungal pathogenicity.

\section{Zearalenone}

Zearalenone (ZEN), [6-(10-hydroxy-6-oxo-trans-1-undecenyil)-resorcylic acid lactone], is an estrogenic mycotoxin produced by numerous Fusarium species during or post-harvest storage in corn, wheat and other cereals (Pittet, 1998). The ZEN-infected feed stuff causes reproductive disorder in swine, sheep, and other farm animals which includes vulva enlargement, pseudopregnancy, embryo loss and reduced litter size (Farnworth and Trenholm, 1983; Green et al., 1990; Long and Diekman, 1986; Zinedine et al., 2007). Although the estrogenic effect of ZEN in humans remain controversial, biochemical data indicates that it also has adverse 
effects on humans. It is reported that ZEN contaminated grain-based food may cause hyperestrogenism and precocious pubertal development in children (Massart and Saggese, 2009; Szuetz et al., 1997). Moreover, ZEN is reported to bind human estrogen receptors (Miksicek, 1994) and observed to stimulate the growth of the human breast cancer cell lines (Makela et al., 1994). Apart from its estrogenicity, ZEN is found to have genotoxic effects in mice and a suspected carcinogen (Coe et al., 1992; Grosse et al., 1997; Pfohl-Leszkowicz et al., 1995).

The biological detoxification of ZEN is one of the safest alternatives to eliminate the potential risks and health implications to humans, animals and plants (Karlovsky, 1999). The biological role of ZEN was evaluated from two different perspectives, in $\mathrm{F}$. graminearum which is a ZEN producer as functional geneknock-out analysis studies (Gaffoor et al., 2005; Gaffoor and Trail, 2006; Kim et al., 2005) and in the detoxifier G. roseum by the pioneering work of (Elsharkawy and Abulhajj, 1988). Later gene-knock-out and gene-cloned studies were carried out by several researchers (Kakeya et al., 2002; Takahashi-Ando et al., 2002; Utermark and Karlovsky, 2007). Gaffoor and co-worker inoculated wheat heads and found out that ZEN disrupted mutants were as virulent as their wild-type counterparts. However, in gene expression studies they found out that ZEA1 and ZEA2 (ZEA1 and ZEA2 are genes responsible for ZEN biosynthesis in F. graminearum) expression was high in grain colonization and resource protection (Gaffoor et al., 2005). Moreover Kim and co-workers proposed that ZEN helps the fungus in adverse survival conditions. Other studies in fact, proved that ZEN is not a virulence factor for the fungus but that its role in substrate colonization and interference competition is more pronounced (Gaffoor et al., 2005; Utermark and Karlovsky, 2007). The late production of ZEN in field and storage conditions is welldocumented (Martins and Martins, 2002; Sutton, 1982). The mycoparasite G. roseum is a promising biocontrol agent and reviewed by (Papavizas, 1985). The ecological role of ZEN was never tested before in the root colonization of maize and wheat infected by F. graminearum. However, several other authors have used G. roseum as a promising biological control agent. Vakili (1992) reported that G. roseum suppressed the Fusarium moniliforme growth on corn in vitro and in field trials. Knudsen (1994) observed great effects of the mycoparasite against B. sorokiniana in infected seedlings and isolated the endophyte from distal and proximal root segments. Burgess et al. (1997) tested the impact of the 
mycoparasite against Botrytis cinerea on chickpea. Xue (2003) investigated Clonostachys rosea on field pea root rot disease and recorded vast disease suppression. The root colonization of barley and wheat seedlings with the mycoparasite G. roseum against F. culmorum was investigated by few researchers (J ensen et al., 2000; Knudsen et al., 1995), who reported it to cause significant disease suppression in root rot diseases. The focus of all these studies was mostly to screen the biocontrol agent or to investigate the antagonistic potential of mycoparasitic fungi against several seed and soil-borne pathogens.

\section{Aim of the work}

In order to understand the ecological role of ZEN, we co-inoculated G. roseum wild-type and a non-detoxifying $G$. roseum Zes2 deficient mutant with F. graminearum in maize and wheat roots. These studies facilitate the biological significance of ZEN in ecological environment to its producer. The reduction of ZEN would explain the consequences on the interference and the competition as well as on the substrate colonization for its competitor.

The pathogenicity and mycotoxin productivity of different F. graminearum strains could explain the choice of the pathogen to infect different hosts in diverse environmental conditions. Moreover, a comparison of different strains would further elaborate the biological role of several mycotoxins on maize and wheat ears as well as their accumulation. To investigate the susceptibility of different plant parts for several F. graminearum strains, experiments with maize and wheat ears as well as roots were conducted in greenhouse and field trials.

The biological role of the two important trichothecenes deoxynivalenol (DON) and nivalenol (NIV) on wheat and maize root infection would facilitate how important these toxins are in causing infection on different hosts. DON and NIV knock-out strains of $F$. graminearum were used in greenhouse trials with maize and wheat to explain their specific role in initiation of disease and virulence. 


\section{References}

1. Bai, G. H., A. E. Desjardins, and R. D. Plattner. 2002. Deoxynivalenolnonproducing Fusarium graminearum causes initial infection, but does not cause disease spread in wheat spikes. Mycopathologia 153:91-98.

2. Bennett, J. W. 1983. Differentiation and secondary metabolism in mycelial fungi. In J.W.Bennett and A.Ciegler (ed.), Secondary metabolism and differentiation in fungi. Marcel Dekker, New York, NY.1-32.

3. Bennett, J. W. 1995. From molecular genetics and secondary metabolism to molecular metabolites and secondary genetics. Canadian J ournal of BotanyRevue Canadienne de Botanique 73:S917-S924.

4. Bergstrom, G. C. and E. J. Shields. 2002. Atmospheric spore dispersal and regional epidemiology of the Fusarium head blight fungus. Phytopathology 92:S93 (Abstr.).

5. Bode, H. B., B. Bethe, R. Hofs, and A. Zeeck. 2002. Big effects from small changes: Possible ways to explore nature's chemical diversity. Chembiochem 3:619-627.

6. Bruins, M. B. M., I. Karsai, J. Schepers, and C. H. A. Snijders. 1993. Phytotoxicity of deoxynivalenol to wheat tissue with regard to in vitro selection for Fusarium head blight resistance. Plant Science 94:195-206.

7. Burgess, D. R., T. Bretag, and P. J. Keane. 1997. Biocontrol of seed-borne Botrytis cinerea in chickpea with Gliocladium roseum. Plant Pathology 46:298-305.

8. Bushnell, W. R., B. E. Hazen, and C. Pritsch. 2003. Histology and physiology of Fusarium head blight. In Fusarium Head Blight of Wheat and Barley (Leonard, K.J .and Bushnell, W.R., eds). St.Paul, MN: APS Press44-83.

9. Chandler, E. A., D. R. Simpson, M. A. Thomsett, and P. Nicholson. 2003. Development of PCR assays to Tri7 and Tri13 trichothecene biosynthetic genes, and characterisation of chemotypes of Fusarium graminearum, Fusarium culmorum and Fusarium cerealis. Physiological and Molecular Plant Pathology 62:355-367.

10. Charoenpornsook, K., J. L. Fitzpatrick, and J. E. Smith. 1998. The effect of four mycotoxins on the mitogen stimulated proliferation of bovine peripheral blood mononuclear cells in vitro. Mycopathologia 143:105-111.

11. Coe, J. E., K. G. Ishak, J. M. Ward, and M. J. Ross. 1992. Tamoxifen prevents induction of hepatic neoplasia by zeranol, an estrogenic food contaminant. Proceedings of the National Academy of Sciences of the United States of America 89:1085-1089. 
12. D'Mello, J. P. and A. M. C. Macdonald. 1997. Mycotoxins. Animal Feed Science and Technology 69:155-166.

13. D'Mello, J. P. F., C. M. Placinta, and A. M. C. Macdonald. 1999. Fusarium mycotoxins: A review of global implications for animal health, welfare and productivity. Animal Feed Science and Technology 80:183-205.

14. Desjardins, A. E. and T. M. Hohn. 1997. Mycotoxins in plant pathogenesis. Molecular Plant-Microbe Interactions 10:147-152.

15. Desjardins, A. E., T. M. Hohn, and S. P. McCormick. 1993. Trichothecene biosynthesis in Fusarium species - Chemistry, genetics, and significance. Microbiological Reviews 57:595-604.

16. Desjardins, A. E. and R. H. Proctor. 2001. Biochemistry and genetics of Fusarium toxins. In B.A.Summerell, J.F.Leslie, D.Backhouse, W.L.Bryden, and L.W. Burgess (ed.), Fusarium. Paul E. Nelson Memorial Symposium50-69.

17. Dill-Macky, R. and R. K. Jones. 2000. The effect of previous crop residues and tillage on Fusarium head blight of wheat. Plant Disease 84:71-76.

18. Elsharkawy, S. and Y. J. Abulhajj. 1988. Microbial cleavage of zearalenone. Xenobiotica 18:365-371.

19. Eriksen, G. S. 2003. Metabolism and toxicity of trichothecenes. Doctoral thesis, Swedish University of Agricultural Science.Uppsala (available at: http:// diss-epsilon. slu. se/ archive/ 00000287/ 01/ Thesis. pdf).

20. Eudes, F., A. Comeau, S. Rioux, and J. Collin. 2000. Phytotoxicity of eight mycotoxins associated with Fusarium in wheat head blight. Canadian J ournal of Plant Pathology-Revue Canadienne de Phytopathologie 22:286-292.

21. Farnworth, E. R. and H. L. Trenholm. 1983. The metabolism of the mycotoxins zearalenone and its effects on the reproductive tracts of young male and female pigs. Canadian J ournal of Animal Science 63:967-975.

22. Gaffoor, I., D. W. Brown, R. Plattner, R. H. Proctor, W. H. Qi, and F. Trail. 2005. Functional analysis of the polyketide synthase genes in the filamentous fungus Gibberella zeae (Anamorph Fusarium graminearum). Eukaryotic Cell 4: 1926-1933.

23. Gaffoor, I. and F. Trail. 2006. Characterization of two polyketide synthase genes involved in zearalenone biosynthesis in Gibberella zeae. Applied and Environmental Microbiology 72:1793-1799.

24. Goswami, R. S. and H. C. Kistler. 2004. Heading for disaster: Fusarium graminearum on cereal crops. Mol. Plant Pathol. 5:515-525.

25. Green, M. L., M. A. Diekman, J. R. Malayer, A. B. Scheidt, and G. G. Long. 1990. Effect of pre-pubertal consumption of zearalenone on puberty and subsequent reproduction of gilts. J ournal of Animal Science 68:171-178.

26. Grosse, Y., L. ChekirGhedira, A. Huc, S. ObrechtPflumio, G. Dirheimer, H. Bacha, and A. PfohlLeszkowicz. 1997. Retinol, ascorbic acid and alpha- 
tocopherol prevent DNA adduct formation in mice treated with the mycotoxins ochratoxin A and zearalenone. Cancer Letters 114:225-229.

27. Gutleb, A. C., E. Morrison, and A. J. Murk. 2002. Cytotoxicity assays for mycotoxins produced by Fusarium strains: A review. Environmental Toxicology and Pharmacology 11:309-320.

28. Hagler, W. M., N. R. Towers, C. J. Mirocha, R. M. Eppley, and W. M. Bryden. 2001. Zearalenone: mycotoxin or mycoestrogen ? In B.A.Summerell, J.F.Leslie, D.Backhouse, W. L.Bryden, and L.W.Burgess (ed.), Fusarium. Paul E. Nelson Memorial Symposium321-331.

29. Harris, L. J., A. E. Desjardins, R. D. Plattner, P. Nicholson, G. Butler, J. C. Young, G. Weston, R. H. Proctor, and T. M. Hohn. 1999. Possible role of trichothecene mycotoxins in virulence of Fusarium graminearum on maize. Plant Disease 83: 954-960.

30. Ito, Y., K. Ohtsubo, K. Ishii, and Y. Ueno. 1986. Effects of nivalenol on pregnancy and fetal development in mice. Mycotoxin Research 2:71-77.

31. Jensen, B., I. M. B. Knudsen, and D. F. Jensen. 2000. Biological seed treatment of cereals with fresh and long-term stored formulations of Clonostachys rosea: Biocontrol efficacy against Fusarium culmorum. European J ournal of Plant Pathology 106:233-242.

32. Kakeya, H., N. Takahashi-Ando, M. Kimura, R. Onose, I. Yamaguchi, and H. Osada. 2002. Biotransformation of the mycotoxin, zearalenone, to a nonestrogenic compound by a fungal strain of Clonostachys sp. Biosci. Biotechnol. Biochem. 66:2723-2726.

33. Karlovsky, P. 1999. Biological detoxification of fungal toxins and its use in plant breeding, feed and food production. Natural Toxins 7:1-23.

34. Khera, K. S., D. L. Arnold, C. Whalen, G. Angers, and P. M. Scott. 1984. Vomitoxin (4-Deoxynivalenol) - Effects on Reproduction of Mice and Rats. Toxicology and Applied Pharmacology 74:345-356.

35. Kim, Y. T., Y. R. Lee, J. M. J in, K. H. Han, H. Kim, J. C. Kim, T. Lee, S. H. Yun, and Y. W. Lee. 2005. Two different polyketide synthase genes are required for synthesis of zearalenone in Gibberella zeae. Molecular Microbiology 58:1102-1113.

36. Knudsen, I. M. B. 1994. Biological control of seed borne diseases. PhD thesis. $\mathrm{PhD}$ thesis. Frederiksberg: The Royal Veterinary and Agricultural University.

37. Knudsen, I. M. B., J. Hockenhull, and D. F. Jensen. 1995. Biocontrol of seedling diseases of barley and wheat caused by Fusarium culmorum and Bipolaris sorokiniana - Effects of selected fungal antagonists on growth and yield components. Plant Pathology 44:467-477.

38. Lee, T., D. W. Oh, H. S. Kim, J. Lee, Y. H. Kim, S. H. Yun, and Y. W. Lee. 2001. Identification of deoxynivalenol- and nivalenol-producing chemotypes of Gibberella zeae by using PCR. Applied and Environmental Microbiology 67:2966-2972. 
39. Lewandowski, S. and W. R. Bushnell. 2001. Development of Fusarium graminearum on floret surfaces of field-grown barley. National Fusarium Head Blight Forum Proceedings. East Lansing: Michigan State University128.

40. Logrieco, A., G. Mule, A. Moretti, and A. Bottalico. 2002. Toxigenic Fusarium species and mycotoxins associated with maize ear rot in Europe. European J ournal of Plant Pathology 108:597-609.

41. Long, G. G. and M. A. Diekman. 1986. Characterization of effects of zearalenone in swine during early pregnancy. American J ournal of Veterinary Research 47: 184-187.

42. Maier, F. J., T. Miedaner, B. Hadeler, A. Felk, S. Salomon, M. Lemmens, H. Kassner, and W. Schafer. 2006. Involvement of trichothecenes in fusarioses of wheat, barley and maize evaluated by gene disruption of the trichodiene synthase (Tri5) gene in three field isolates of different chemotype and virulence. Mol. Plant Pathol. 7:449-461.

43. Makela, S., V. L. Davis, W. C. Tally, J. Korkman, L. Salo, R. Vihko, R. Santti, and K. S. Korach. 1994. Dietary estrogens act through estrogen receptormediated processes and show no antiestrogenicity in cultured breast cancer cells. Environmental Health Perspectives 102:572-578.

44. Martins, M. L. and H. M. Martins. 2002. Influence of water activity, temperature and incubation time on the simultaneous production of deoxynivalenol and zearalenone in corn (Zea mays) by Fusarium graminearum. Food Chemistry 79:315-318.

45. Massart, F. and G. Saggese. 2009. Oestrogenic mycotoxin exposures and precocious pubertal development. International Journal of Andrology 33:369-376.

46. McLean, M. 1996. The phytotoxicity of Fusarium metabolites: An update since 1989. Mycopathologia 133:163-179.

47. McMullen, M., R. Jones, and D. Gallenberg. 1997. Scab of wheat and barley: A re-emerging disease of devastating impact. Plant Disease 81:1340-1348.

48. Miksicek, R. J. 1994. Interaction of naturally occurring nonsteroidal estrogens with expressed recombinant human estrogen receptor. Journal of Steroid Biochemistry and Molecular Biology 49:153-160.

49. Miller, J . D. 1994. Epidemiology of Fusarium diseases of cereals. In: Miller J D and Trenholm HL (eds) Mycotoxins in Grain: Compounds other than Aflatoxin. Eagan Press, St. Paul, MN, USA19-36.

50. Munkvold, G. P. 2003. Epidemiology of Fusarium diseases and their mycotoxins in maize ears. European J ournal of Plant Pathology 109:705-713.

51. Ohtsubo, K., J. C. Ryu, K. Nakamura, N. Izumiyama, T. Tanaka, H. Yamamura, T. Kobayashi, and Y. Ueno. 1989. Chronic toxicity of nivalenol in female mice - A 2-year feeding study with Fusarium nivale Fn 2B-molded rice. Food and Chemical Toxicology 27:591-598. 
52. Papavizas, G. C. 1985. Trichoderma and Gliocladium - Biology, ecology, and potential for biocontrol. Annual Review of Phytopathology 23:23-54.

53. Parry, D. W., P. J enkinson, and L. Mcleod. 1995. Fusarium ear blight (Scab) in small grain cereals - A review. Plant Pathology 44:207-238.

54. Pestka, J.J. 2008. Mechanisms of deoxynivalenol-induced gene expression and apoptosis. Food Additives and Contaminants Part A-Chemistry Analysis Control Exposure \& Risk Assessment 25: 1128-1140.

55. Pestka, J. J. and A. T. Smolinski. 2005. Deoxynivalenol: Toxicology and potential effects on humans. Journal of Toxicology and Environmental Health-Part B-Critical Reviews 8:39-69.

56. Pfohl-Leszkowicz, A., L. Chekir-Ghedira, and H. Bacha. 1995. Genotoxicity of zearalenone, an estrogenic mycotoxin: DNA adduct formation in female mouse tissues. Carcinogenesis 16:2315-2320.

57. Pittet, A. 1998. Natural occurrence of mycotoxins in foods and feeds : An update review. Rev. Med. Vet. (Toulouse) 149:479-492.

58. Pritsch, C., G. J. Muehlbauer, W. R. Bushnell, D. A. Somers, and C. P. Vance. 2000. Fungal development and induction of defense response genes during early infection of wheat spikes by Fusarium graminearum. Molecular Plant-Microbe Interactions 13: 159-169.

59. Reid, L. M. and R. I. Hamilton. 1996. Effects of inoculation position, timing, macroconidial concentration, and irrigation on resistance of maize to Fusarium graminearum infection through kernels. Canadian J ournal of Plant Pathology-Revue Canadienne de Phytopathologie 18:279-285.

60. Reubel, G. H., M. Gareis, and W. M. Amselgruber. 1987. Cytotoxicity evaluation of mycotoxins. Mycotoxin Research 3:85-86.

61. Ribichich, K. F., S. E. Lopez, and A. C. Vegetti. 2000. Histopathological spikelet changes produced by Fusarium graminearum in susceptible and resistant wheat cultivars. Plant Disease 84:794-802.

62. Richard, J. L. 2007. Some major mycotoxins and their mycotoxicoses - An overview. International J ournal of Food Microbiology 119:3-10.

63. Rocha, O., K. Ansari, and F. M. Doohan. 2005. Effects of trichothecene mycotoxins on eukaryotic cells: A review. Food Additives and Contaminants 22:369-378.

64. Ryu, J. C., K. Ohtsubo, N. Izumiyama, K. Nakamura, T. Tanaka, H. Yamamura, and Y. Ueno. 1988. The acute and chronic toxicities of nivalenol in mice. Fundamental and Applied Toxicology 11:38-47.

65. SCF . 1999. Opinion on Fusarium toxins. Part 1: Deoxynivalenol (DON). (Scientific Committee for Food) (available at: http:/ / www.europa.eu.int/ comm/ food/ fs/ sc/scf/ out44_en.pdf). 
66. SCF . 2002. Opinion on Fusarium toxins. Part 6: Group evaluation of T-2 toxin, HT-2 toxin, nivalenol and deoxynivalenol. (Scientific Committee for Food) (available at: http:/ / www. europa.eu.int/comm/food/fs/ sc/ scf/ out123_en. pdf).

67. Schaafsma, A. W., L. Tamburic-Ilinic, J. D. Miller, and D. C. Hooker. 2001. Agronomic considerations for reducing deoxynivalenol in wheat grain. Canadian J ournal of Plant Pathology-Revue Canadienne de Phytopathologie 23:279-285.

68. Shimada, T. and M. Otani. 1990. Effects of Fusarium Mycotoxins on the growth of shoots and roots at germination in some J apanese wheat cultivars. Cereal Research Communications 18:229-232.

69. Smith, D. R. and D. G. White. 1988. Diseases of corn. In: Sprague GF and Dudley JW (eds) Corn and Corn Improvement, 3rd edn ( $p p$ 687-766) Agronomy Series No. 18. Am. Soc. Agronomy, Madison, WI, USA.

70. Sutton, J. C. 1982. Epidemiology of wheat head blight and maize ear rot caused by Fusarium graminearum. Canadian Journal of Plant Pathology 4: 195.

71. Szuetz, P., A. Mesterhazy, G. Y. Falkay, and T. Bartok. 1997. Early telearche symptoms in children and their reaction to zearalenone contamination in food stuffs. Cereals Research Communication 25:429-436.

72. Takahashi-Ando, N., M. Kimura, H. Kakeya, H. Osada, and I. Yamaguchi. 2002. A novel lactonohydrolase responsible for the detoxification of zearalenone: Enzyme purification and gene cloning. Biochem.J. 365:1-6.

73. The commission of the European communities. 2006. Commission regulation (EC) No 1881/ 2006 of 19 December 2006 setting maximum levels for certain contaminants in foodstuffs. Official J ournal of the European Union L 364/ 5.

74. Trail, F., I. Gaffoor, and S. Vogel. 2005. Ejection mechanics and trajectory of the ascospores of Gibberella zeae (anamorph Fuarium graminearum). Fungal Genetics and Biology 42:528-533.

75. Tryphonas, H., F. Iverson, Y. So, E. A. Nera, P. F. Mcguire, L. Ogrady, D. B. Clayson, and P. M. Scott. 1986. Effects of deoxynivalenol (vomitoxin) on the humoral and cellular immunity of mice. Toxicology Letters 30: 137-150.

76. Ueno, Y. 1985. The toxicology of mycotoxins. Crc Critical Reviews in Toxicology 14:99-132.

77. Utermark, J. and P. Karlovsky. 2007. Role of zearalenone lactonase in protection of Gliocladium roseum from fungitoxic effects of the mycotoxin zearalenone. Applied and Environmental Microbiology 73:637-642.

78. Vakili, N. G. 1992. Biological seed treatment of corn with mycopathogenic fungi. Journal of Phytopathology-Phytopathologische Zeitschrift 134:313323. 
79. Vidnes, A., B. Paulsen, and C. Bergsten. 2003. Report from SCOOP task3.2.10 "Collection of occurrence data of Fusarium toxins in food and assessment of dietary intake by the population of EU member states"-Subtask II: zearalenone.[Online.]

http:/ / europa. eu. int/ comm/ food/ fs/ scoop/ task3210. pdf.

80. Wakulinski, W. 1989. Phytotoxicity of the secondary metabolites of fungi causing wheat head fusariosis (Head Blight). Acta Physiologiae Plantarum 11:301-306.

81. Wang, H., S. F. Hwang, F. Eudes, K. F. Chang, R. J. Howard, and G. D. Turnbull. 2006. Trichothecenes and aggressiveness of Fusarium graminearum causing seedling blight and root rot in cereals. Plant Pathology 55:224-230.

82. Wang, Y. Z. and J. D. Miller. 1998. Effects of Fusarium graminearum metabolites on wheat tissue in relation to FHB resistance. Journal of Phytopathology 122: 118-125.

83. Wannemacher, R. J., L. Stanley, and M. D. Wiener. 2000. Trichothecenes mycotoxins. In: Zajtchuk $\mathrm{R}$, editor.Medical aspects of chemical and biological warfare. Washington, DC: Department of the Army656-676.

84. Xue, A. G. 2003. Biological control of pathogens causing root rot complex in field pea using Clonostachys rosea strain ACM941. Phytopathology 93:329335.

85. Zinedine, A., J. M. Soriano, J. C. Molto, and J. Manes. 2007. Review on the toxicity, occurrence, metabolism, detoxification, regulations and intake of zearalenone: An oestrogenic mycotoxin. Food and Chemical Toxicology 45: 118. 


\title{
Chapter 2: Role of zearalenone in the interaction of F. graminearum with $\mathbf{G}$. roseum in vitro and in wheat and maize rhizosphere
}

\author{
Awais Ahmed and Petr Karlovsky \\ Molecular Phytopathology \& Mycotoxin Research Unit, Georg-August University, \\ Goettingen, Germany.
}

\section{Abstract}

The estrogenic mycotoxin zearalenone (ZEN) is produced by several Fusarium species including $\mathrm{F}$. graminearum. Its common occurrence in cereal grains and maize pose severe health risk to humans and farm animals. We investigated the ecological role of ZEN in maize and wheat root infection and mixed colony incubations. G. roseum, which detoxifies ZEN and its knock-out non-detoxifying mutants (Zes2 mutant) were generated and tested earlier in our research group. We found that colonies of $\mathrm{G}$. roseum wild-type and Zes2 mutant started to grow the same time on the pink-covered surface of $F$. graminearum on YES medium, but the wild-type colony size increased a multiple-fold and appeared as hollowed spots or digested pits on the pathogen surface. The pigmentation changed at the borders from light-pale to dark-orange over several days of incubation. However, the Zes2 mutant colony diameter stayed compact, no significant expansion was measured. In the root colonization trials, 5 aggressive $F$. graminearum strains were co-inoculated with G. roseum wild-type and Zes2 mutants on maize and wheat roots. Significant differences were observed among the seedlings inoculated with $F$. graminearum and co-inoculated with $\mathrm{G}$. roseum wild-type and Zes2 mutants. The co-inoculated seedlings with G. roseum wild-type and pathogen showed increased emergence, larger yields in root and shoot indexes and less symptoms of disease, Whereas, the Zes2 mutant resulted in partial protection in maize and wheat seedlings. We conclude that ZEN is involved in substrate colonization and the detoxification leaves its producer in a hostile ecological environment, Zes2 protects the resource from other competitors. 


\section{Introduction}

F. graminearum (Schwabe) is one of the most toxigenic plant-pathogenic species which belongs to the genus Fusarium. The fungus overwinters as a saprophyte on diverse ecological niches world-wide and becomes a necrophyte on a wide host range (Sutton, 1982; Parry et al., 1995; Munkvold, 2003). F. graminearum is a destructive pathogen which causes head blight and root rot of wheat and barley (Goswami and Kistler, 2004) and ear and root rot of maize (Logrieco et al., 2002). The fungus produces the type B trichothecenes deoxynivalenol (DON) and nivalenol (NIV) and its derivatives, as well as the estrogenic mycotoxin zearalenone (ZEN). ZEN contains estrogenic activity and can be a significant contaminant of maize, barley, wheat and other cereals (Hagler et al., 2001; Vidnes et al., 2003). Besides grain contamination, it causes hyperestrogenism in pigs, and reproductive problems in experimental and farm animals (Desjardins and Proctor, 2001). ZEN has several other derivatives, such as $\alpha$-zearalenol, that have stronger estrogenic activity than ZEN (Shier et al., 2001). The biological detoxification of ZEN may be the safest and most useful alternative to prevent grain contamination and health risk to human and farm animals (Karlovsky, 1999). Until now, the ecological role of ZEN is a topic of great speculation. ZEN does not benefit the producer in plant infection (Gaffoor et al., 2005) but functional gene expression studies revealed that it may aid pathogen to grain colonization and may play a role in adverse survival conditions (Gaffoor et al., 2005; Kim et al., 2005). The wheat head inoculation with ZEN deficient mutants of $F$. graminearum produced the same amount of disease as their progenitors (Gaffoor et al., 2005).

G. roseum, a mycoparasite, is recognized as a promising biocontrol agent against several soil and seed-borne pathogens (Papavizas, 1985; Knudsen et al., 1995). G. roseum has lactonase activity which can hydrolyze ZEN (Elsharkawy and Abulhajj, 1988; Takahashi-Ando et al., 2002; Utermark and Karlovsky, 2007). Utermark and Karlovsky (2007) hypothesized that ZEN is an agent of interference competition and aids the pathogen in substrate colonization. The gene-knock-out studies revealed that $G$. roseum non-detoxifying mutants (Zes2) was found retarded in growth at a ZEN of $20 \mu \mathrm{g} / \mathrm{ml}$ amended on Czapek-Dox-medium. However, G. roseum wild-type showed no inhibition against ZEN (Utermark and Karlovsky, 2007). Moreover, the inhibitory effects were also found on ten filamentous fungi against ZEN in 
concentrations as low as $2 \mu \mathrm{g} / \mathrm{ml}$ on Malt-Extract-Agar-medium (MEA). The complete inhibition was measured at $\geq 10 \mu \mathrm{g} / \mathrm{ml}$ with an exception of $\mathrm{G}$. roseum. The ecological significance of ZEN to the producer and its detoxification leave some crucial questions open in natural environments. We investigated the ZEN producer $F$. graminearum and its detoxifier $G$. roseum in different combinations in maize and wheat root infection. The objectives of this study were 1 . to understand the enzymatic activity (ZEN detoxification) on co-incubation of $G$. roseum wildtype and its Zes2 mutant colonies with F. graminearum, 2. to investigate the role of ZEN in substrate colonization or protection against its competitor in root colonization trials on maize and wheat seed infection.

\section{Material and Methods}

\section{Fungal strains, culture conditions and media}

The mycoparasite G. roseum DSM62726 was obtained from the Deutsche Sammlung von Mikroorganismen und Zellkulturen (Braunschweig, Germany) and the G. roseum non-detoxifying Zes2 mutant was generated in our research group (Utermark and Karlovsky, 2007). Five pre-screened aggressive F. graminearum strains F4, F10, F11, F26 and F47 (original codes, host and geographical origin are listed in Table 1: chapter 2). In mixed colony incubation and inhibition bioassays, the F. graminearum strain F26 was used, because of large ZEN production.

G. roseum was cultivated on GM7 medium with slight modification from (Karlovsky, 1994) using $1.5 \%$ agar for solidifying. Later on $50 \mu$ of spore suspension was spread on the solidified medium and incubated for three weeks on 25ㅇ for sporulation. Spores were harvested by flooding ca. $5 \mathrm{ml}$ sterile water on the culture plates. Spores were kept as stock on $15 \%$ glycerol at $-80^{\circ} \mathrm{C}$. F. graminearum strains were cultivated on mung bean medium described by (Bai and Shaner, 1996). In brief, 50 $\mu \mathrm{l}$ of spores' suspension were injected in $250 \mathrm{ml}$-Erlenmeyer flasks containing 100 $\mathrm{ml}$ of medium and shaken for 4 days at $28^{\circ} \mathrm{C}$ at $200 \mathrm{rpm}$ on a shaker (Innova 44, New Brunswick Scientific, USA). Spore count was determined by adding a few $\mu \mathrm{l}$ into a Thoma counting chamber and followed up by a germination test (CFU test). 
In vitro bioassays were performed on two different media. Potato Dextrose Broth Agar (PDA) contained $24 \mathrm{~g}$ of potato dextrose broth dissolved in 1 litre of distilled water; the pH was adjusted to 5.6. Yeast Extract Sucrose Agar (YES) consisted of 20 $\mathrm{g}$ of yeast extract, $150 \mathrm{~g}$ of sucrose and $\mathrm{MgSO}_{4} .7 \mathrm{H}_{2} \mathrm{O} 0.5 \mathrm{~g}$ dissolved in $885 \mathrm{ml}$ of distilled water. Both media were autoclaved at $121^{\circ} \mathrm{C}$ for 20 minutes and solidified with $1.5 \%$ agar.

\section{Mixed colony incubation bioassays}

Bioassays were conducted on $9 \mathrm{~cm} \varnothing$ petri dishes filled with either YES or PDA solidified media. Two treatments $F$. graminearum $+G$. roseum Wt $(F g+G r W T)$ and F. graminearum $+\mathrm{G}$. roseum non-detoxifier mutant $(\mathrm{Fg}+\mathrm{GrZes} 2)$ were used. On solidified media either YES or PDA, 100 microliter of F. graminearum spores (100 cfu) were spread with L-shaped glass rod. The petri dishes were dried for ca. 5 minutes under the sterile bench and this was followed by spreading the 100 microliter of G. roseum wild-type or Zes2 mutant spores (30 cfu). Moreover, independent control dishes were prepared for the mycoparasite and pathogen, inoculated with $\mathrm{G}$. roseum alone or with $\mathrm{F}$. graminearum. Four replicates were set for each treatment. Plates were incubated at $25^{\circ} \mathrm{C}$ and monitored every day starting from day 3 to day 5 . A dilution series was done using a100 microliter of spore suspension, either pathogen or mycoparasite on PDA petri dishes, to determine the colony forming unit (cfu). The cfu for both $\mathrm{G}$. roseum and $\mathrm{F}$. graminearum was determined prior to the bioassays conduct.

\section{Inhibition zone bioassays}

The bioassays were conducted on $9 \mathrm{~cm} \varnothing$ solidified PDA plates. Both F. graminearum and G. roseum were grown on PDA plates for a week. Two millimeter $\varnothing$ agar discs were obtained from the outer edge of mycelia grown on PDA for a week. The discs were inverted (facing downwards) and placed opposite each other on plates. The treatments were denoted as F. graminearum $+\mathrm{G}$. roseum Wt $(\mathrm{Fg}+\mathrm{GrWT})$, F. graminearum $+\mathrm{G}$. roseum non-detoxifier mutant $(\mathrm{Fg}+\mathrm{GrZes} 2)$ and $\mathrm{F}$. graminearum (control). The plates were incubated at $25^{\circ} \mathrm{C}$ and 
monitored every second day for two weeks. Five replicates were made for each treatment.

\section{Seedling growth conditions}

The hybrid maize cv. 'KABANAS' and wheat $\mathrm{CV}$. 'CUBUS' were kindly provided by KWS SAAT AG, Einbeck, Germany and Lochow-Petkus GmbH, Bergen, Germany respectively. Both cultivars are designated by their producers as moderately susceptible to $F$. graminearum infection. Both cultivars were grown during pathogenicity tests in a greenhouse at approximately $24 \pm 3^{\circ} \mathrm{C}$ with $16 \mathrm{~h}$ supplemental light (400W Philips bulbs with PIA technology) with the relative humidity between $40-60 \%$ Seedlings were watered daily and fertilized in weekly intervals with NPK (15-11-15).

\section{Aggressiveness test on maize and wheat root}

The same procedure was used for both maize and wheat. Five pre-screened F. graminearum strains (F4, F10, F11, F26 and F47) were co-inoculated with either G.roseum wild-type or Zes2 mutant on maize and wheat. The treatment codes were designated as $\mathrm{Fg}$ (only F. graminearum), Fg+GrWT (F. graminearum + G. roseum wild-type), Fg+GrZes2 (F. graminearum + G. roseum non-detoxifier Zes2 mutant) and control (water inoculated seeds). One seed was planted in each $4.5 \times 5$ $\mathrm{cm} \varnothing$ plastic pot in a mixture of plant substrate + sand ratio (2:1) a. The seed inoculation method was modified from (Knudsen et al., 1995). Each seed was placed on a piece of paper towel $\varnothing \mathrm{ca} .0 .5 \mathrm{~cm}$ and spore suspension was carefully released on to it. The seed was inoculated for treatment ( $\mathrm{Fg}+\mathrm{GrWT})$ with a mixture of $F$. graminearum $\left(1.5 \times 10^{5}\right)$ and $G$. roseum $\left(10^{6}\right)$ set in one mililitre, positive control (Fg) F. graminearum $\left(1.5 \times 10^{5}\right)$ and negative control (sterile tap water) was inoculated on seeds. The Disease Index (DI) or rating was scored at harvest, using the disease scale described by (Knudsen et al., 1995) with slight modification where 0 - no disease symptoms; 1 - brown pin point lesions on the coleoptile and/ or roots; 2 - extended browning of the coleoptile and/ or browning/ reddening of the roots; 3 - dead coleoptile and/ or extended browning/reddening of the roots; and 4 - browning extending beyond the coleoptile on the above-ground plant parts 
or dead plant or nongerminated kernel. The percentage of plants was calculated after one week and final seedling stand was evaluated at harvest. Other parameters which were fresh shoot and root weight indexes, seedling height, survival and final seedling stand were also recorded. The experiment was conducted in randomized block design on greenhouse benches. Each treatment had 10 replicates and the experiment was repeated twice under the same conditions.

\section{Fungal DNA extraction (CTAB method)}

The F. graminearum DNA was extracted from lyophilized root or shoot, following the upscaled protocol for DNA-extraction from plant material published by Brandfass and Karlovsky (2008), modified as follows: The samples were ground and weighed. Because of high variability in root and shoot samples ca. $10 \mathrm{mg}$ of sample were blended in a $2 \mathrm{ml}$ reaction tube with $1 \mathrm{ml}$ CTAB-buffer. The total DNA of 10 mg plant material was dissolved in $50 \mu \mathrm{LTE}$ buffer. The DNA quantity was checked by agarose gel electrophoresis before DNA quantification with Real- time PCR.

\section{F. graminearum DNA quantification by Real- time PCR}

Real-time PCR protocol was carried out as described (Brandfass and Karlovsky, 2008) based on primers specific for F. graminearum described by (Nicholson et al., 1998). The PCR amplification was done according to cited work (Brandfass and Karlovsky, 2008), with the following modifications: The PCR was performed by using Absolute $^{\text {TM }}$ Blue QPCR SYBR GREEN Fluorescein Mix (Thermo Start TM DNAPolymerase, $3 \mathrm{mM} \mathrm{MgCl}$, dNTP Mixture, SYBR Green I, and $10 \mathrm{nM}$ Fluorescein, Abgene Limited, United Kingdom). Calibration curve was carried out with purified F. graminearum DNA in $0.5 \mathrm{pg}, 5 \mathrm{pg}, 50 \mathrm{pg}$ and $500 \mathrm{pg}$ added to healthy plant shoot or root matrix. PCR product ratios were converted to DNA concentrations (picograms of total DNA), then to microgram per kilogram $(\mu \mathrm{g} / \mathrm{kg})$ plant material. 


\section{Statistical analysis}

Colony diameter was determined in centimeter $(\mathrm{cm})$ with the image analysis software ImageJ (http:// rsb.info.nih.gov/ nih-image) after scanning the petri dishes at a resolution of $400 \mathrm{dpi}$. The data were analyzed with student t-test at the significant level $P \varangle 0.05$. The pathogenicity test data from maize and wheat was analyzed by One Way ANOVA with Multiple Comparison Procedures (Fisher LSD Method) at $P \leq 0.05$ using software program Sigma plot version 11.0 (Systat Software, Inc. USA).

\section{Results}

\section{Mixed colony incubation bioassays}

The co-incubation of mycoparasite $G$. roseum wild-type and its non-detoxifying Zes2 mutant with F. graminearum was evaluated on YES and PDA solidified media. The F. graminearum colonies appeared as white-small-cushion in 12 hours post inoculation and light pink surface covered the whole petri dish area until the next day. The $G$. roseum colonies started to grow on $F$. graminearum covered surface after 24 hours post inoculation in both G. roseum wild-type and Zes2 mutant. Significant differences were observed in continue incubation of the mycoparasite on pathogen. When $G$. roseum wild-type was co-inoculated, the colony size increased multiple-fold as hollowed spots or digested pits appeared on pinkcovered surface. Moreover, a change of pigmentation was also monitored on the edges of colonies in G. roseum wild-type from light pale to dark-orange over days intervals. However, Zes2 mutant colony diameter stayed compact as no large expansion was measured and a mild change of pigments was observed with a longer incubation (Fig. 1 and 2). No significant differences were observed when the mycoparasite and the pathogen were co-incubated on PDA solidified medium. The PDA petri dishes were soon after co-incubation covered with mixture of pink and white fluffy cushions. They expanded fast on the whole surface area witnessed for the pathogen presence. No observations were recorded because of over-grown mycelia. 


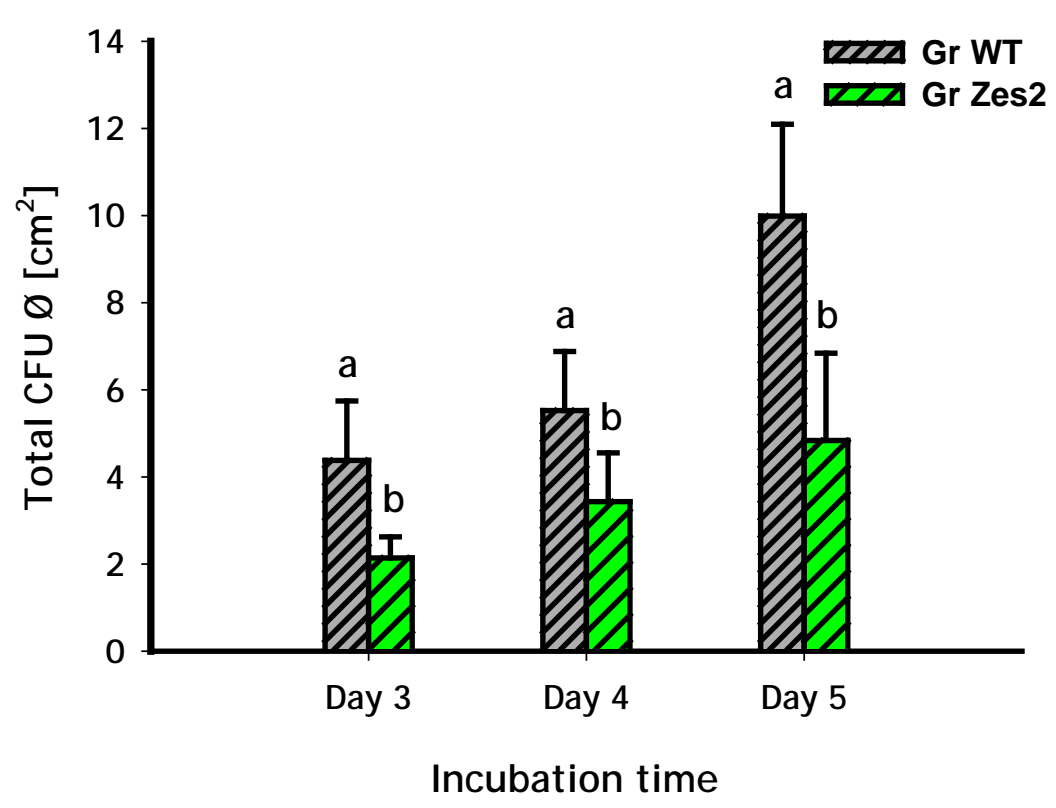

Figure 1. Mixed colony growth of $G$. roseum and $F$. graminearum on YES (Yeast Extract Sucrose Agar). Gr WT (G. roseum wild-type) and Gr Zes2 (G. roseum non-detoxifying Zes2 mutant) co-incubated with F. graminearum (F26) at $25^{\circ} \mathrm{C}$ for 5 days. Capped columns with same letters are not significantly different with student t-test at $(P<0.001)$. Each column is the pooled mean of 4 replicates with SD. 

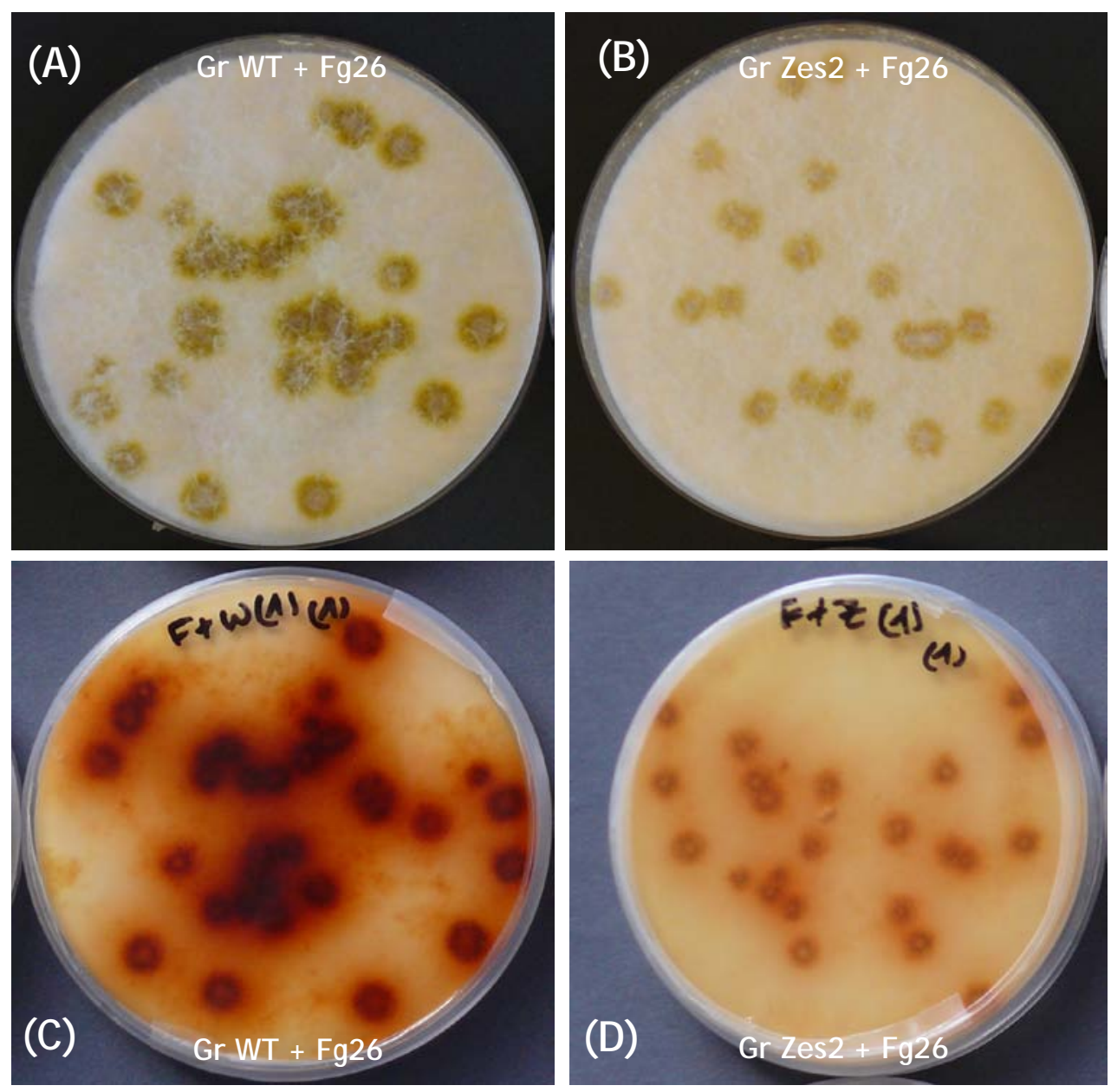

Figure 2. Mixed colony growth of $G$. roseum and $F$. graminearum on YES (Yeast Extract Sucrose Agar). Upper row open-lid petri dishes filled with YES (A) G. roseum wild-type $+F$. graminearum, (B) G. roseum non-detoxifying Zes2 mutant $+F$. graminearum. Lower row bottom-exposed (C) G. roseum wild-type $+F$. graminearum, (D) G. roseum non-detoxifying Zes2 mutant $+\mathrm{F}$. graminearum co-incubated for for 5 days at $25^{\circ} \mathrm{C}$. 


\section{Inhibition zone bioassays}

There were no significant differences observed when G. roseum (Gr WT) was tested against F. graminearum (F26). The F26 mycelia grew swiftly over the surface of 9 $\mathrm{cm} \varnothing$ solidified PDA plates (within 12 hours after the discs were placed). However, Gr (WT) and its non-detoxifying mutant Gr (Zes2) did not seem to grow as fast as its competitor (Fig. 3). The first contact was monitored on day 8 of co-incubation when F26 approached the growing mycelia of either Gr (WT) or Gr (Zes2). Afterwards F26 stopped growing further into both G. roseum strains. Both G. roseum behaved similarly.
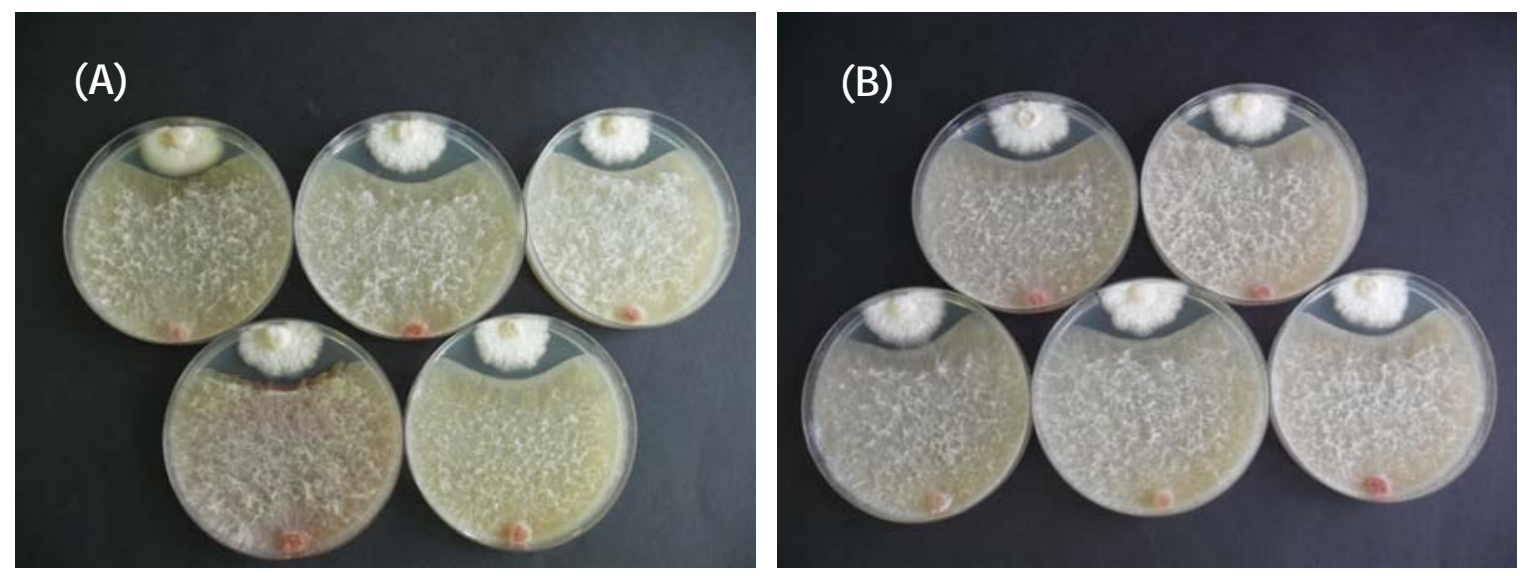

Figure 3. Growth inhibition of $G$. roseum and $F$. graminearum on PDA (Potato Dextrose Broth Agar). Gr WT (G. roseum wild type) and Gr Zes2 (G. roseum non-detoxifying mutant) co-cultivated with F. graminearum (F26). Open-lid PDA petri plates, (A) Gr WT against Fg26, (B) Gr Zes2 against Fg26 on 10-day-co-incubation at $25^{\circ} \mathrm{C}$. 


\section{Ecological role of ZEN and maize seedlings protection with G. roseum}

The maize seedlings inoculated with $\mathrm{F}$. graminearum strains and co-inoculated with G. roseum non-detoxifying mutant ( $\mathrm{Gr}$ Zes2) suffered serious intensity of disease. However, co-inoculation with G. roseum wild type ( $\mathrm{Gr}$ Wt) showed a higher germination rate. Most of the seeds failed to germinate or showed complete blight symptoms on germinated seedlings. In Gr Zes2 co-inoculated with F. graminearum the seed emergence was partially protected, but effects were much more pronounced when Gr Wt protected the seed which accounted for a greater level of germination. The seedling emergence rate was extremely poor in the $F$. graminearum treated variant compared to both $G$. roseum wild-type and mutant co-inoculated seeds. The seedling survival close to harvest was recorded to be between 30 to $70 \%$ in F. graminearum infected seeds and 50 to $80 \%$ in Fg+ Gr Zes2 co-inoculated. However, an improved rate was scaled in $\mathrm{Fg}+\mathrm{Gr}$ Wt co-inoculation which ranged from 60 to $100 \%$ (Fig. 4).

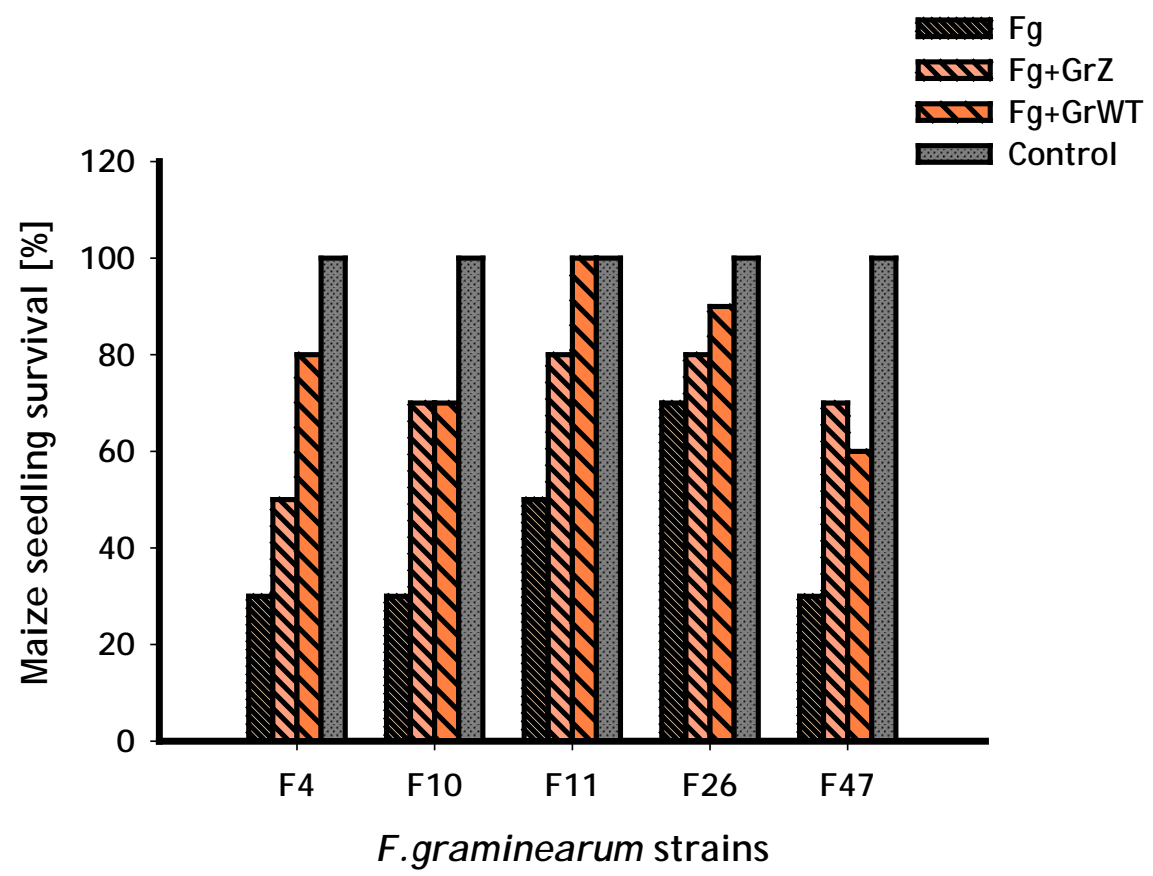

Figure 4. F. graminearum infected maize seedlings protected with G. roseum. Survived seedlings over 4-week-duration represented $F$. graminearum $(\mathrm{Fg})$, F. graminearum + G. roseum (Zes2) non-detoxifying mutant $(\mathrm{Fg}+\mathrm{GrZ}), \quad F$. graminearum+ G. roseum (WT) (Fg+GrWT), water inoculated only (Control). 
(A)

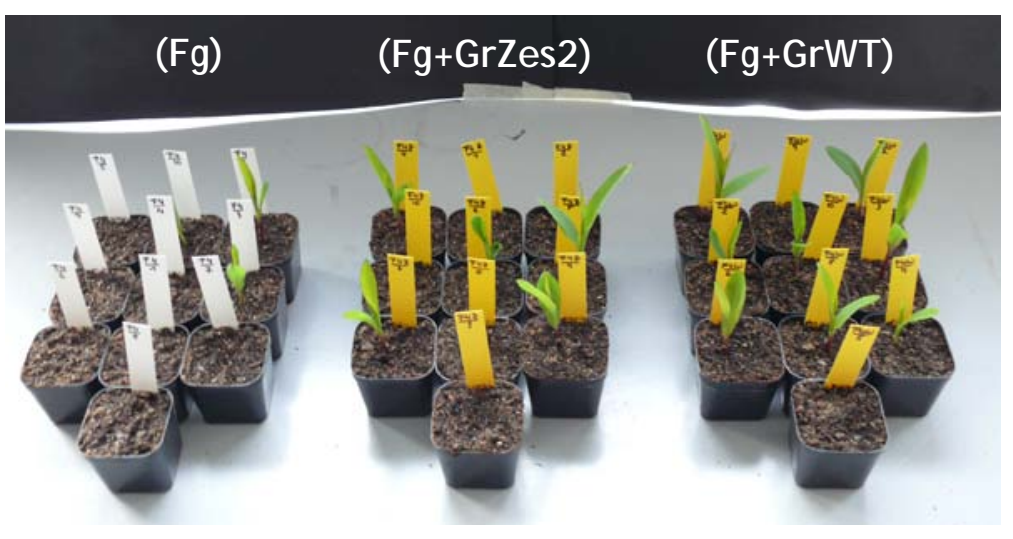

(B)

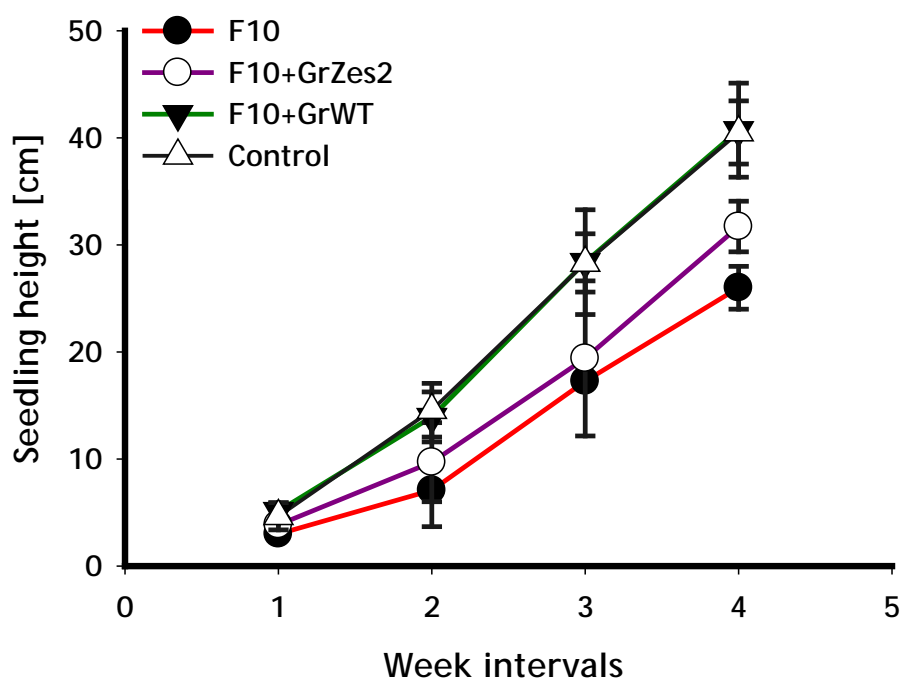

(C)

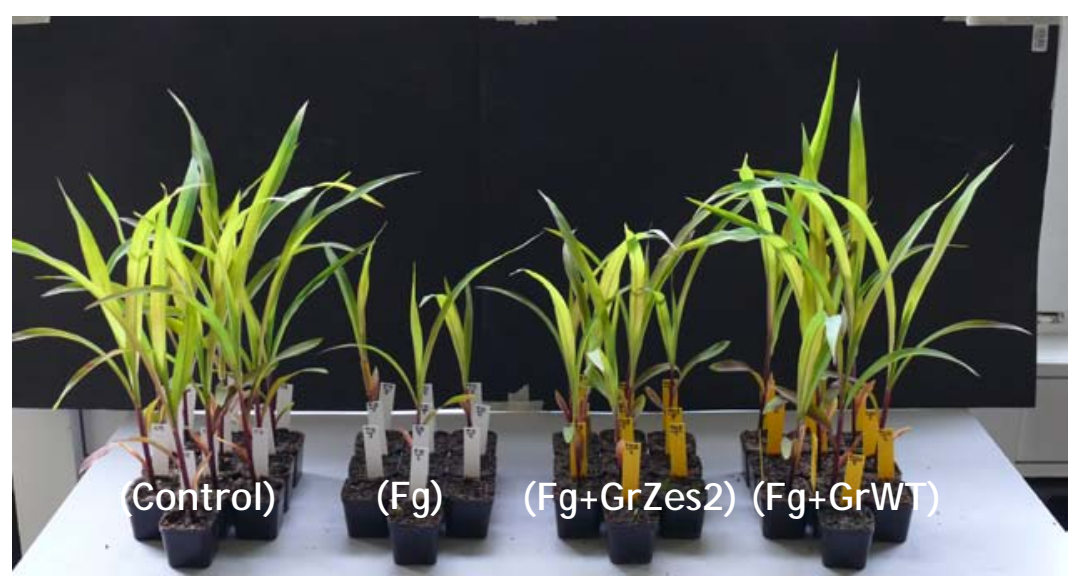

Figure 5. Ecological role of ZEN and maize seedling protection against $F$. graminearum. Different stages are shown for F. graminearum strain (F10). water inoculated (Control), Only $F$. graminearum $(\mathrm{Fg})$, F. graminearum + G. roseum (Zes2) non-detoxifying mutant $(\mathrm{Fg}+\mathrm{GrZ})$, F. graminearum $+\mathrm{G}$. roseum wild-type (Fg+GrWT). roseum(A) 7-day old seedlings after emergence, (B) Seedlings height over week intervals, (C) Seedlings stand after 4week-duration in greenhouse. 
The disease index was scored higher in F. graminearum treated seedlings. Numerous seeds were unable to grow after initial infection and resulted in poor seedling stands in all treatments with $\mathrm{F}$. graminearum strains. Those seedlings which managed to emerge through the seeds, suffered later on with severe infections. Symptoms like seedling blight, browning of coleoptiles and foot rot were frequently observed and increased in severity as the disease progressed. Disease severity was greatest on F. graminearum infected seedlings, where it approached 3.5 on the disease scale ( $0=$ healthy and $4=$ dead). Many seeds also scored 4, which isthe highest disease level, while they failed to germinate during the infection process with the pathogen. The large differences were observed between $\mathrm{Fg}+\mathrm{Gr}$ Zes2 and $\mathrm{Fg}+\mathrm{Gr}$ Wt throughout all treated seedlings. The disease score was relatively pronounced on $\mathrm{Fg}+\mathrm{Gr}$ Zes2, most of the seedlings failed to germinate. This can also be attributed to the inability of the Zes2 mutant to provide adequate protection (Fig. 5). The disease occurred in most of the seedlings since it was initiated in the Fg treated seeds. The ability to colonize the substrate by the mycoparasite looked more convincing in treatments where $\mathrm{G}$. roseum was inoculated with the combinations Fg+ Gr Wt in all tested strains (Fig. 6).

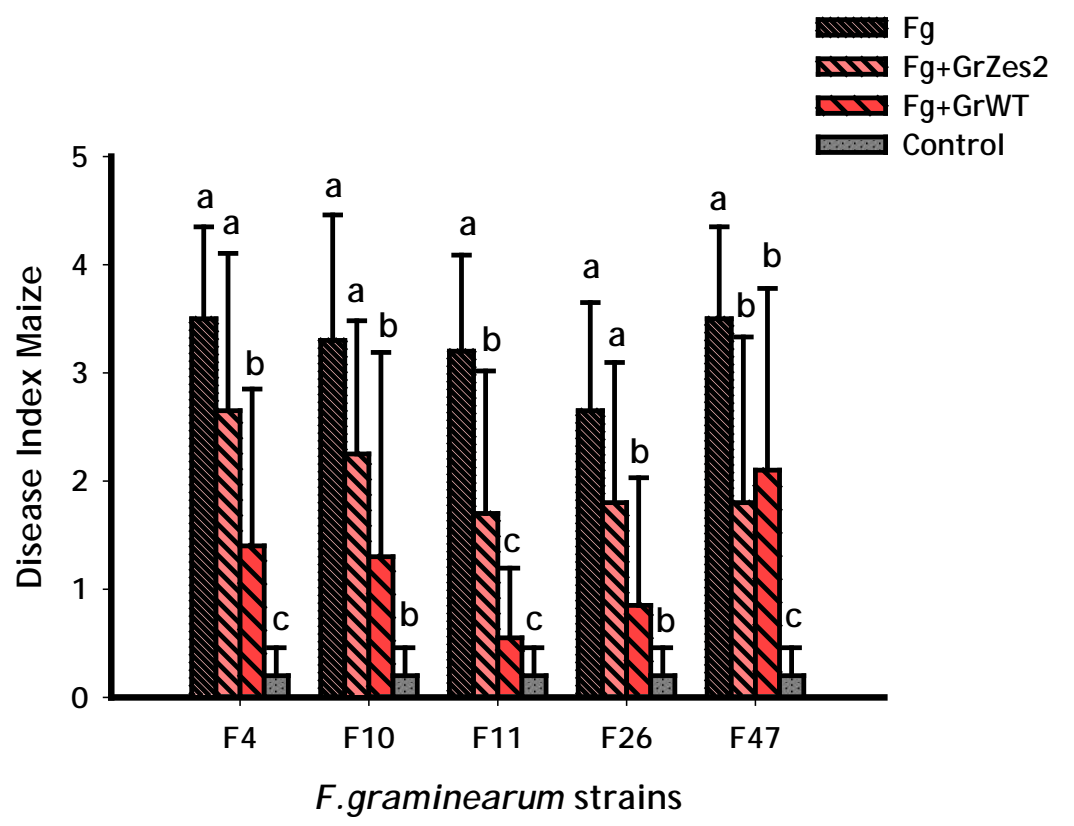

Figure 6. Disease index (DI) on 4-week-old maize seedlings infected by F. graminearum and protected with $\mathrm{G}$. roseum. Numeric with (F) letter represent different F. graminearum strains. DI was scored $0=$ healthy and $4=$ dead (see section Material \& Methods). Bars capped with the same letter are not significantly different based on One Way ANOVA with Multiple Comparison Procedures (Fisher LSD Method) at $P \leq 0.05$. Each bar is averaged from 10 replicates per treatment with standard deviation. 
The infection process affects the seedlings stand in context to height. Large differences were accounted for $F$. graminearum inoculated seeds which were grown to maturity with visible symptoms of disease severity. F. graminearum posed severe aggressiveness at the emergence level that resulted in the poor seedlings height in F. graminearum inoculated plants. However, seedlings stands looked far better in the co-inoculated treatment with $\mathrm{Fg}+\mathrm{Gr}$ Zes2. The co-inoculated seedlings with $\mathrm{Fg}+\mathrm{Gr}$ Wt came to complete maturity just like the healthy seedlings (water inoculated) (Fig. 7A). The height in different $\mathrm{F}$. graminearum inoculated seedlings was measured from 23.6 to $29.6 \mathrm{~cm}$. In comparison, Fg+ Gr Zes2 treated seedlings ranged from 31.7 up $35.1 \mathrm{~cm}$. The $\mathrm{Fg}+\mathrm{Gr}$ Wt co-inoculated seedlings were 37.7 to $40.7 \mathrm{~cm}$ height after 4-weeks, compared to $40.5 \mathrm{~cm}$ in healthy seedlings (water inoculated). The severe disease attack also affected the shoot biomass count, where the seedlings with $F$. graminearum treatments showed an extremely low biomass compared to the controls. The results of $\mathrm{Fg}+\mathrm{Gr}$ Zes2 and $\mathrm{Fg}+\mathrm{Gr}$ Wt in different F. graminearum strains combinations showed significant differences. The fresh biomass accounted 1.3 to $1.7 \mathrm{~g}$ in the $\mathrm{F}$. graminearum inoculated and 1.7 to $2.5 \mathrm{~g}$ in the $\mathrm{Fg}+\mathrm{Gr}$ Zes2 variant. However, Fg+ Gr Wt treated seedlings were greatly protected against Fusarium infection and disease severity and yielded large biomass in all treatments. The biomass was between 2.7 to $3.5 \mathrm{~g}$ compared to $3.5 \mathrm{~g}$ in water treated seedlings (Fig. 7B). The seedling blight affected the shoot biomass indexes in all $\mathrm{F}$. graminearum treatments in comparison to the controls. The visible differences were also observed in $\mathrm{Fg}+\mathrm{Gr}$ Zes2 and $\mathrm{Fg}+\mathrm{Gr} \mathrm{Wt}$, most seedlings co-inoculated with $\mathrm{Fg}+\mathrm{Gr}$ Wt contained a high amount of biomass 4week after inoculation.

F. graminearum was shown to produce larger disease infection on root development than on shoot development. The root rot rating was considered higher than the blight because the disease index was scored on whole plant. Being a seed borne pathogen, F. graminearum remained more destructive on roots than on other plant parts during the disease progression. The F. graminearum inoculated seedlings suffered heavy losses and resulted in poor or little biomass, which ranged from 1 to $1.4 \mathrm{~g}$ with all F. graminearum strains. The co-inoculated Fg+ $\mathrm{Gr}$ Zes2 seedlings displayed extreme weight reduction, which ranged between 1.8 to $2.2 \mathrm{~g}$. However, co-inoculation of $\mathrm{Fg}+\mathrm{Gr}$ Wt yielded substantial biomass indexes and produced a constant range of 2.1 to $2.8 \mathrm{~g}$ compared to $2.9 \mathrm{~g}$ in water treated plants (Fig. 7C). 
(A)

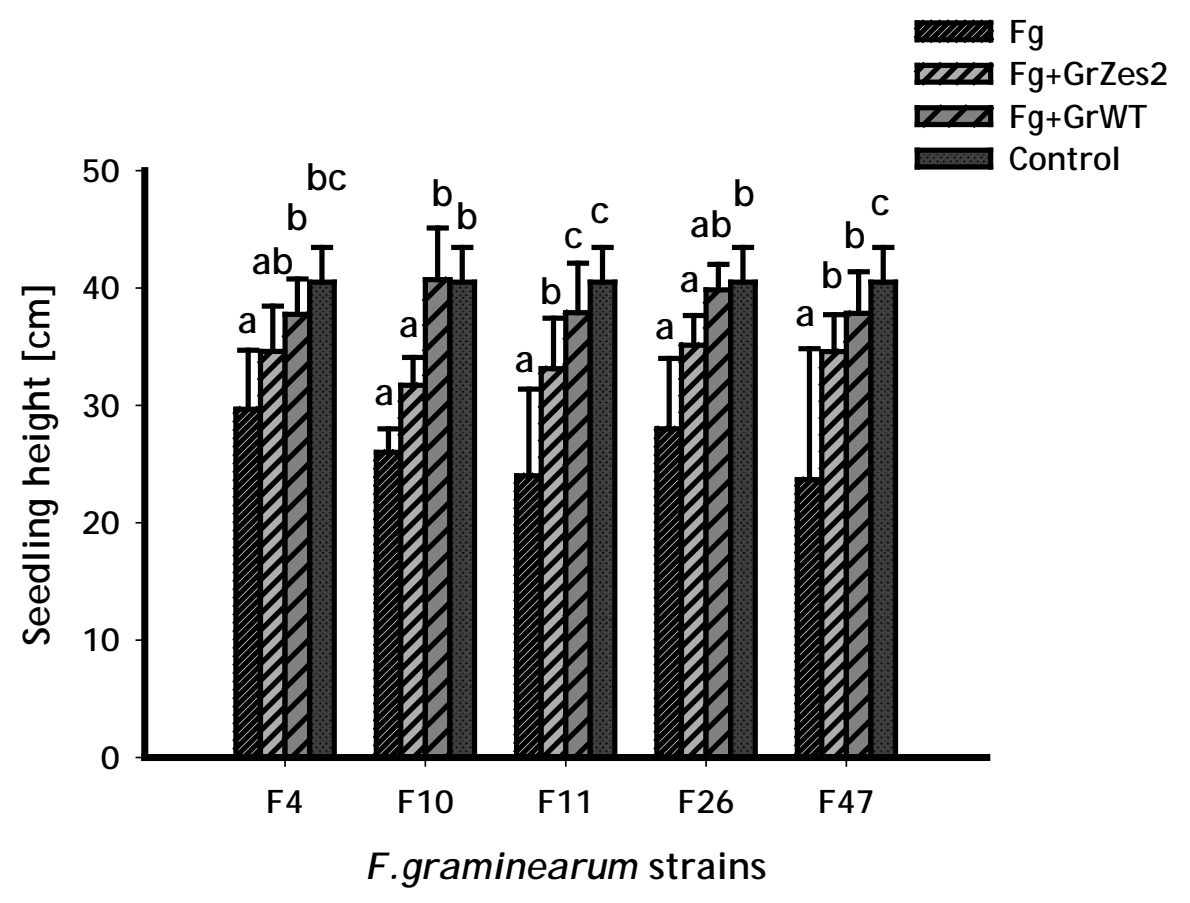

(B)

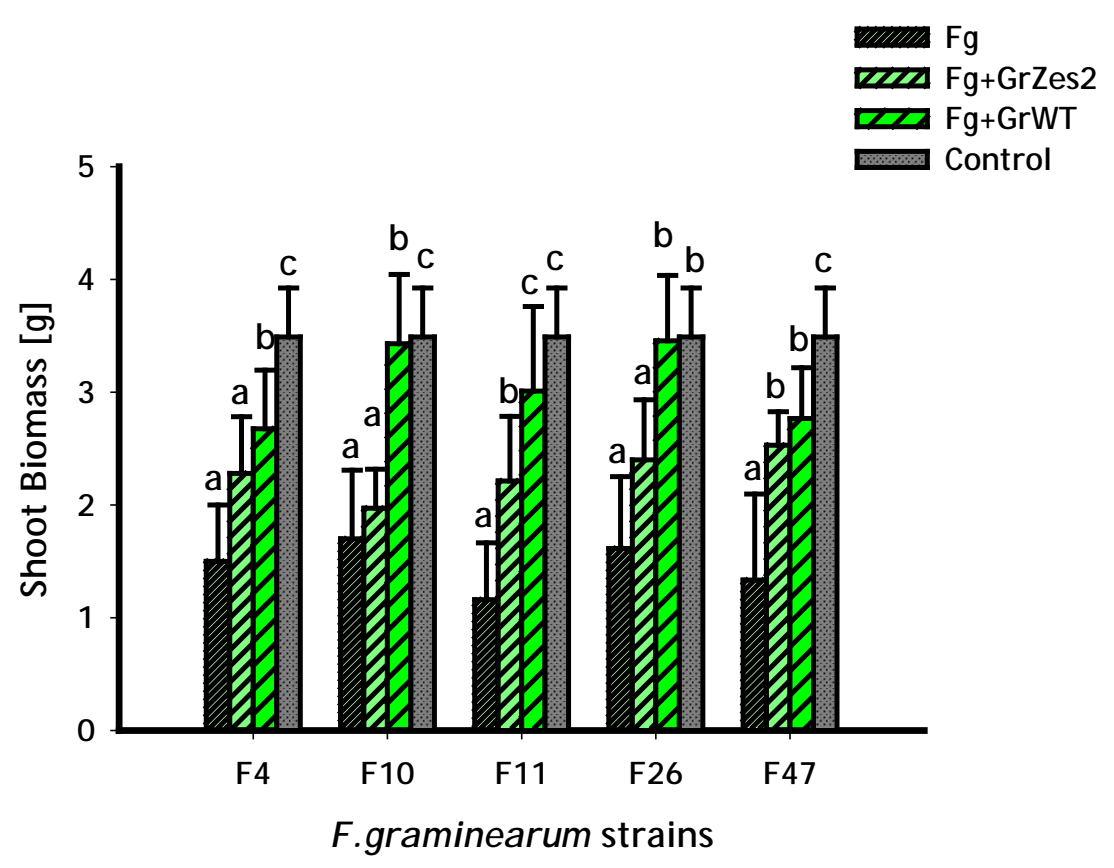


(C)

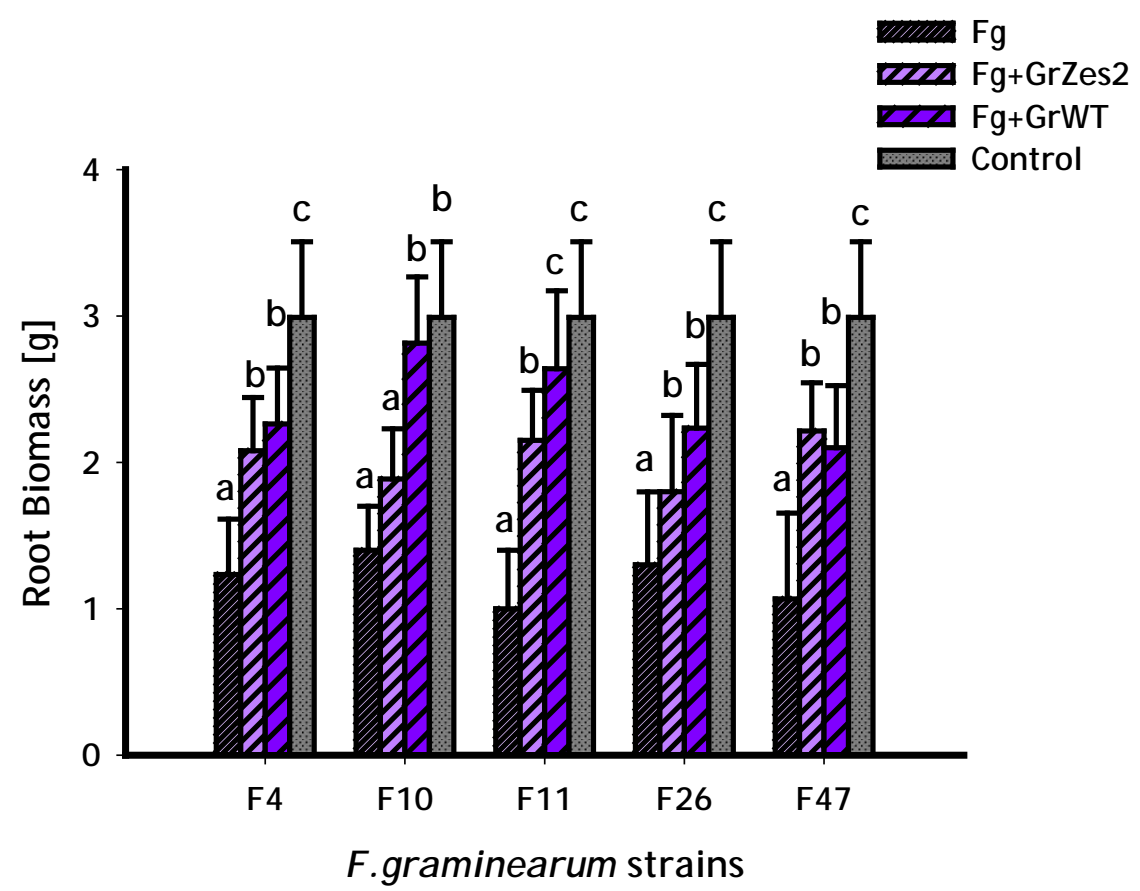

Figure 7. Biological role of $G$. roseum in maize seedling protection against $F$. graminearum. Numeric with (F) letter represents the different $F$. graminearum strains. Different treatments Fg (F. graminearum), Fg+GrZ (F. graminearum+ G. roseum (Zes2) non-detoxifying mutant), Fg+GrWT (F. graminearum+ G. roseum (WT), Control (water inoculated only). (A) Seedlings height, (B) fresh shoot biomass and (C) fresh root biomass on 4-week old greenhouse grown plants. Bars capped with the same letter are not significantly different based on One Way ANOVA with Multiple Comparison Procedures (Fisher LSD Method) at $P \leq 0.05$. Each bar is averaged from 10 replicates per treatment with standard deviation.

\section{Ecological role of ZEN and wheat seedlings protection with G.roseum}

The seedling emergence rate was much better in wheat compared to maize. Large number of seeds was able to germinate at the beginning. The F. graminearum inoculated seeds showed a reduced germination rate compared to other treatments (data not shown). The disease severity was lower on wheat seedlings as compared to maize (Fig. 8A-B). Most of the seedlings showed mild symptoms of disease at the emergence stage; therefore, adequate blights and browning of coleoptiles seemed vital on only F. graminearum inoculated seedlings. There was a negligible account of disease observed on both Fg+ Gr Zes2 and Fg+ Gr Wt inoculated seeds. The disease index scored on Fg treated seeds were from 1.9 to 2.9 on the scale, where $0=$ healthy and $4=$ dead. The disease severity wasslightly lower in $\mathrm{Fg}+\mathrm{Gr}$ Zes2 and ranged 0.7 to 1 . However, almost no or little disease was visible on $\mathrm{Fg}+\mathrm{Gr}$ Wt co- 
inoculated seedlings where range was 0.15 to 0.6 on the scale. There were minor symptoms also on water treated seeds scored as 0.3 traced as back ground infection (Fig. 9).

\section{(A)}

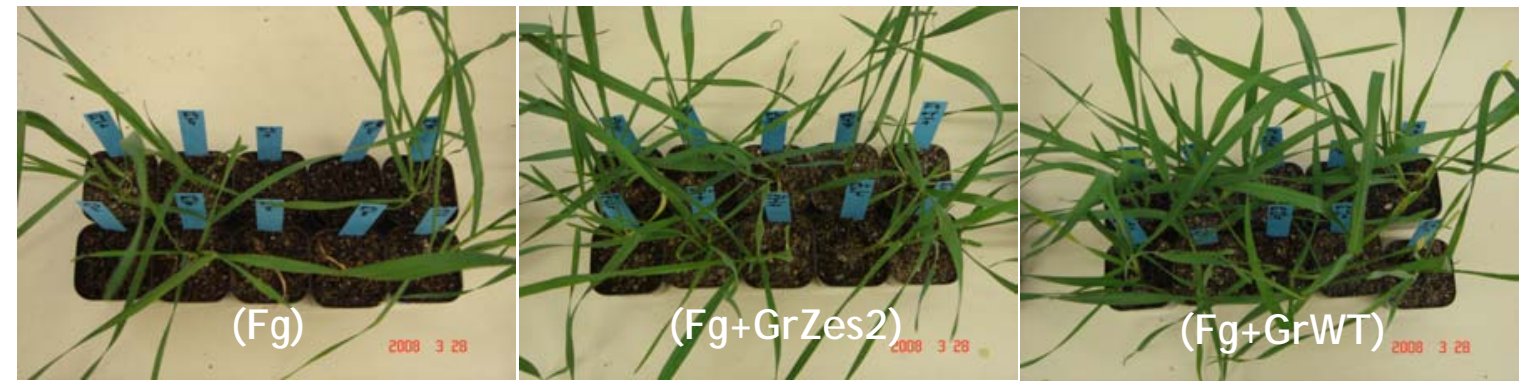

(B)

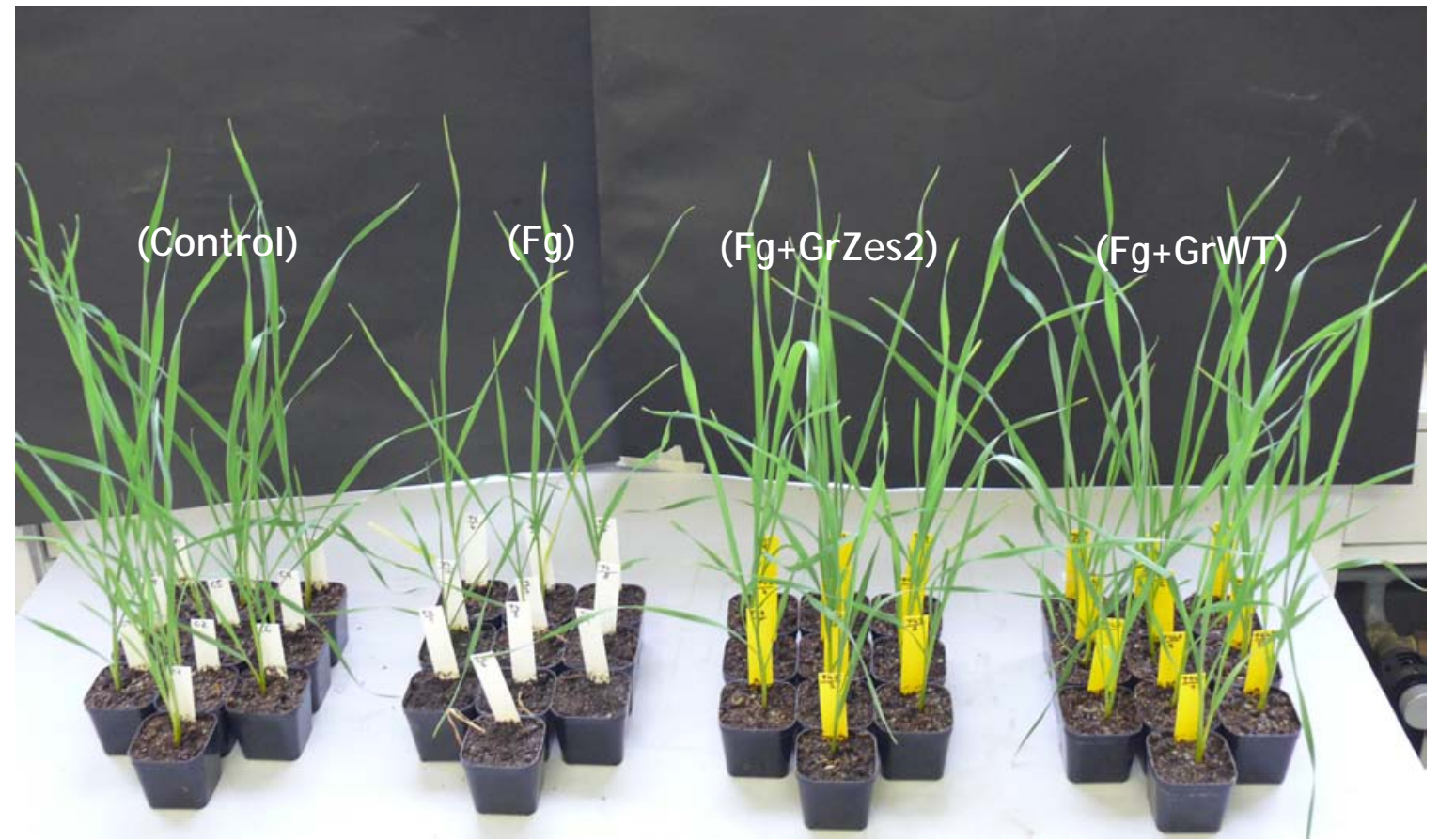

Figure 8. Biological role of $G$. roseum in wheat seedlings protection against $F$. graminearum. (A) 5-week-old seedlings, left to right: $\mathrm{Fg}$ ( $\mathrm{F}$. graminearum), $\mathrm{Fg}+\mathrm{GrZ}$ (F. graminearum + G. roseum (Zes2) non-detoxifying mutant), Fg+GrWT (F. graminearum+ G. roseum (WT). (B) 4-week-old seedlings, left to right: Control (water treated), Fg (F. graminearum), Fg+GrZ (F. graminearum+ G. roseum (Zes2) non-detoxifying mutant), $\mathrm{Fg}+\mathrm{GrWT}$ ( $\mathrm{F}$. graminearum+G. roseum (WT) on greenhouse benches. 


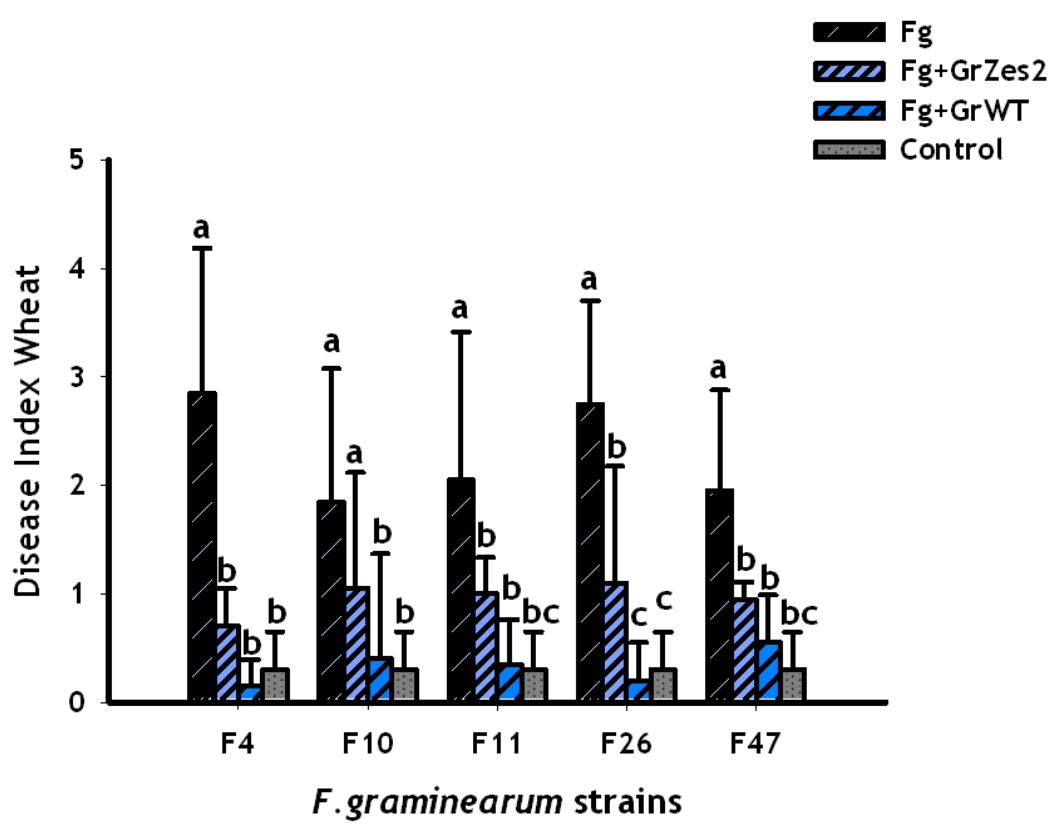

Figure 9. Disease index (DI) on 4-week old wheat seedlings infected with F. graminearum and protected by G. roseum. Numeric with (F) letter represents different F. graminearum strains. DI scored $0=$ healthy and $4=$ dead (see details section material and methods). Bars capped with the same letter are not significantly different based on One Way ANOVA with Multiple Comparison Procedures (Fisher LSD Method) at $P \leq 0 \cdot 05$. Each bar is averaged from 10 replicates per treatment with standard deviation.

There were less significant differences observed in height index. The seedlings showed slight partial ailment and no poor seedlings stand. There were some pronounced differences observed only in $\mathrm{Fg}$ inoculated seedlings height indexes. The height indexes of the $\mathrm{Fg}$ inoculated plants ranged between 23.6 to $29 \mathrm{~cm}$, however the indexes of $\mathrm{Fg}+\mathrm{Gr}$ Zes2 co-inoculated plants ranged between 31.1 to $32 \mathrm{~cm}$. The $\mathrm{Fg}+\mathrm{Gr}$ Wt co-inoculated seedlings ranged from 32.8 to $35 \mathrm{~cm}$ compared to $34.5 \mathrm{~cm}$ in water treated seedlings (Fig. 10A).

The extreme infection was evaluated on shoot indexes where the F. graminearum inoculated plants paid the highest cost to the pathogenicity. On the other hand large differences were also recorded between $\mathrm{Fg}+\mathrm{Gr}$ Zes2 and $\mathrm{Fg}+\mathrm{Gr}$ Wt throughout co-inoculation with different F. graminearum strains. The differences in disease severity were not visible in the first week, however, the disease severity went on to extreme heights as the experiments progressed to the second and third week. The F. graminearum inoculated seedlings appeared almost disease-free on emergence, but the disease came up soon after 7 days and vital effects appeared due to $\mathrm{F}$. graminearum colonization. The pathogen colonization affected the shoot biomass and consequently resulted in reduced weight, which ranged in $F$. graminearum inoculated plants between 0.5 to $0.8 \mathrm{~g}$. Fg+ Gr Zes2 co-inoculated 
seedlings were relatively better and measured between 1.02 to $1.07 \mathrm{~g}$. The $\mathrm{Fg}+\mathrm{Gr}$ Wt co-inoculated seedlings turned out to contain the greatest biomass which was between 1.2 to $1.3 \mathrm{~g}$ compared to $1.2 \mathrm{~g}$ in water treated seedlings (Fig. 10B).

The root rot disease severity affected the root biomass indexes more in $F$. graminearum treated seedlings than the other treatments. The seedlings which suffered root symptoms in particular, showed a reduced biomass count. However, the seedlings co-inoculated with $\mathrm{Fg}+\mathrm{Gr}$ Wt produced a larger amount of biomass than the control. The root weight indexes ranged in $\mathrm{Fg}$ inoculated plants between 0.5 to $0.9 \mathrm{~g}$ and $\mathrm{Fg}+\mathrm{Gr}$ Zes2 co-inoculated seedlings with pathogen between 1.0 to $1.4 \mathrm{~g}$. Moreover, the co-inoculated seedlings with $\mathrm{Fg}+\mathrm{Gr}$ Wt ranged between 1.22 to $1.44 \mathrm{~g}$ compared to 1.32 in water treated seedlings (Fig. 10C). Additionally, when we inoculated wheat seedlings with $\mathrm{G}$. roseum Wt, we recorded an improved emergence rate, larger root systems and the seedlings yielded in greater amount of biomass both in shoot and root indexes compared to water treated seeds (data not shown).

(A)

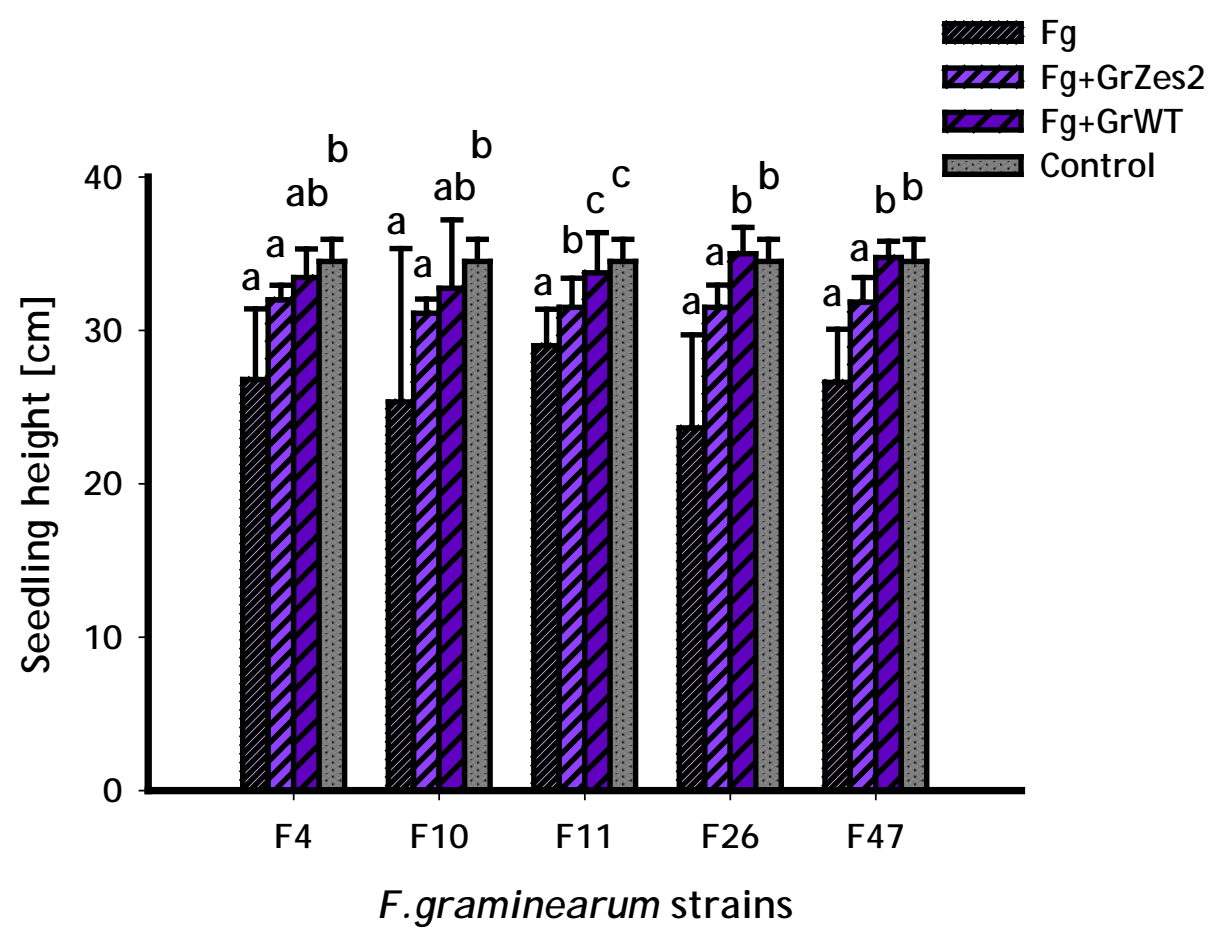


(B)

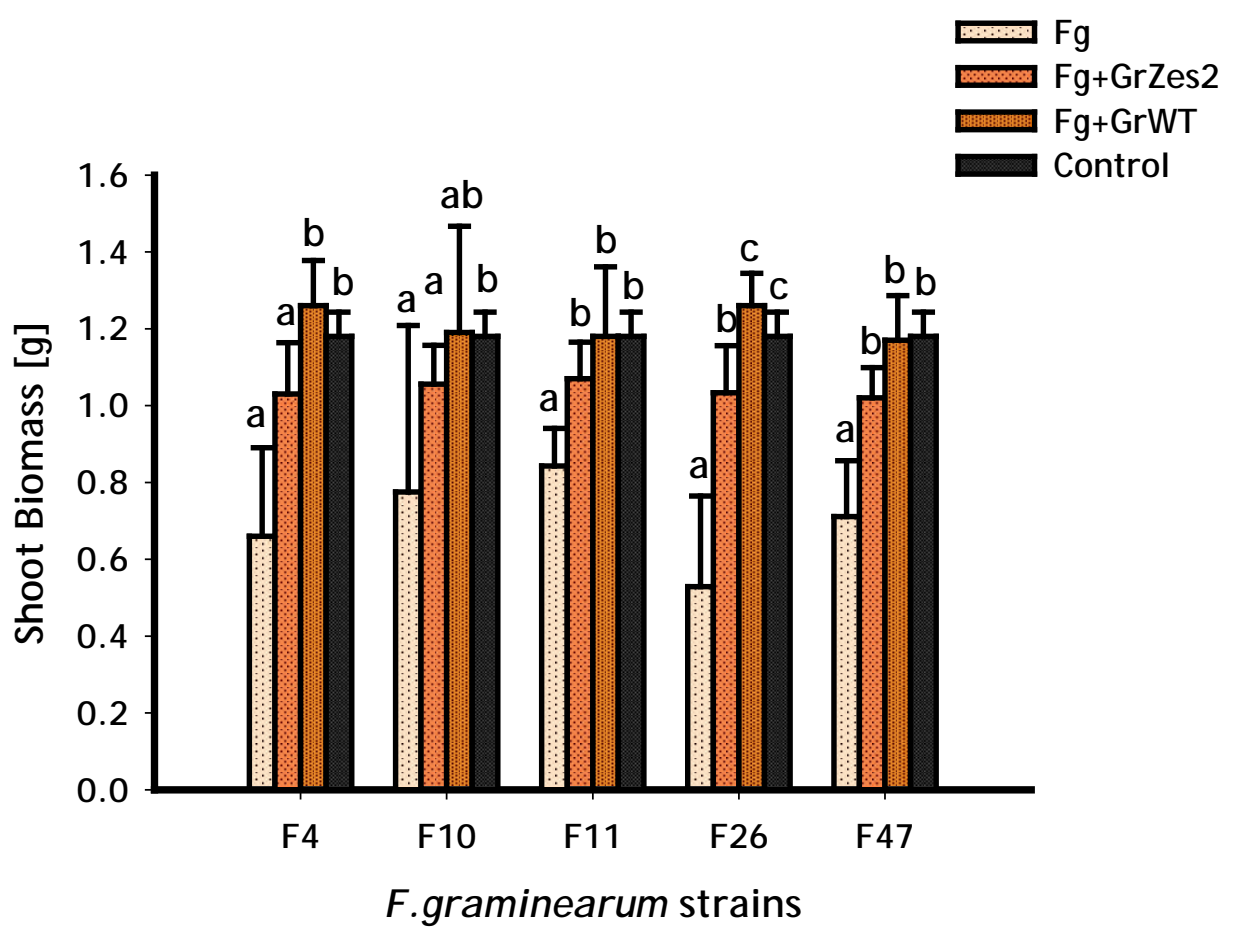

(C)

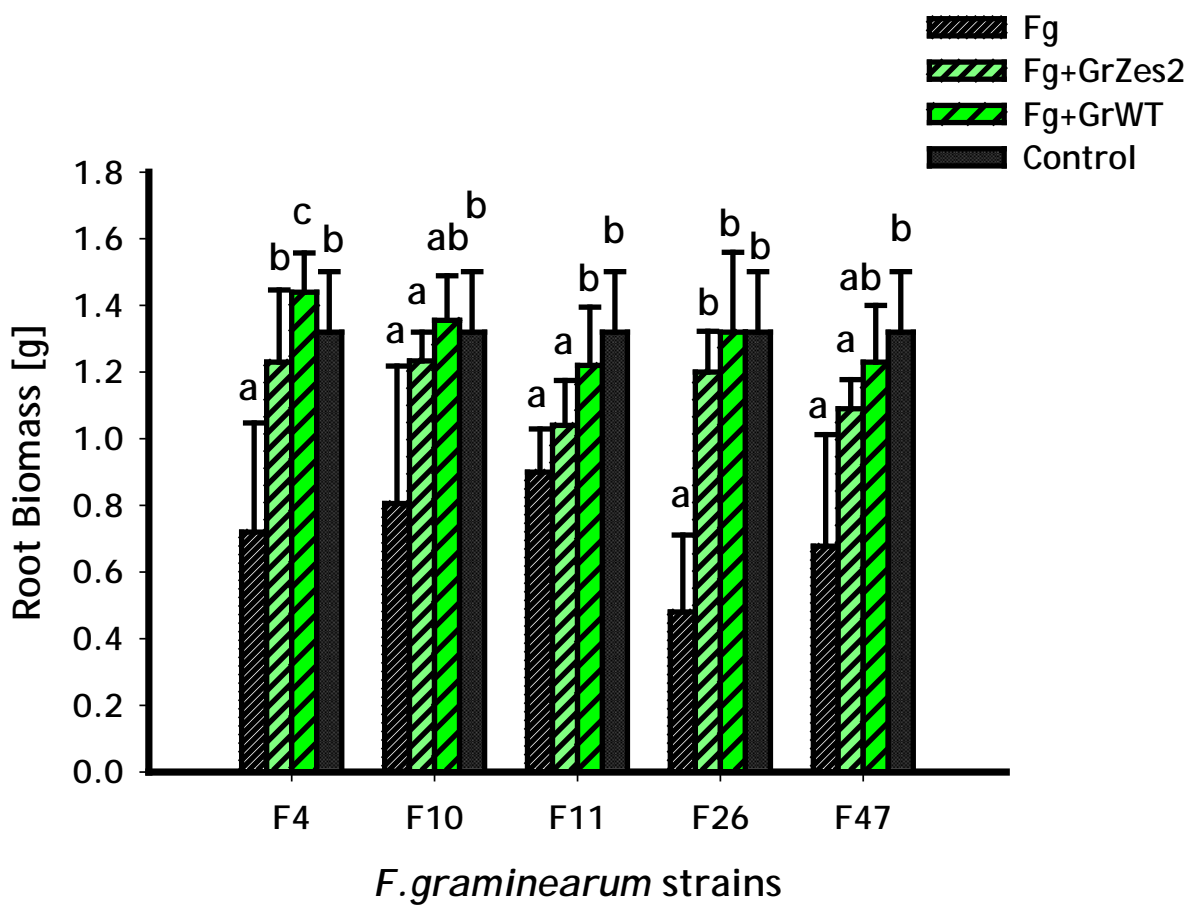

Figure 10. Biological role of $G$. roseum in maize seedlings protection against $F$. graminearum. Numeric with $F$ letter represents different $F$. graminearum strains. Different treatments $\mathrm{Fg}$ ( $\mathrm{F}$. graminearum), $\mathrm{Fg}+\mathrm{GrZ}$ ( $\mathrm{F}$. graminearum $+\mathrm{G}$. roseum (Zes2) non-detoxifying mutant), Fg+GrWT (F. graminearum+ G. roseum (WT), Control (water inoculated only). (A) Seedlings height, (B) Fresh shoot biomass and (C) Fresh root biomass on 4-week-old plants in the greenhouse. Bars capped with the same letter are not significantly different based on One Way ANOVA with Multiple Comparison Procedures (Fisher LSD Method) at $P \leq 0.05$. Each bar is an average of 10 replicates per treatment wit standard deviation. 


\section{Discussion}

The co-occurrence of $G$. roseum and Fusarium species in the same habitat poses some fundamental questions. Utermark and Karlovsky (2007) hypothesized the biological role of ZEN. ZEN aids Fusarium spp. to reduce or inhibit the growth of many filamentous fungi with the exception of G. roseum. G. roseum, which is a mycoparsite, produces lactonase and can hydrolyze ZEN(Elsharkawy and Abulhajj, 1988; Utermark and Karlovsky, 2007). Utermark and Karlovsky (2007) generated knock-out mutants of $G$. roseum and tested the enzymatic detoxification of ZEN on MEA medium. The $G$. roseum wild-type and its generated knock-outs were tested on MEA enriched with synthetic ZEN. We established the in vitro system based on the original ZEN producer (F. graminearum) and its competitor (G. roseum). The mixed colony incubation bioassays revealed that the ZEN producer was ( $F$. graminearum) overcome by its competitor (G. roseum wild-type). However, Zes2 mutant was retarded in growth against ZEN producer. The expansion of colony diameter showed clear differences between G. roseum wild-type and its Zes2 mutant. There was a sharp increase observed in colony diameter of $G$. roseum wild-type which showed hollowed or digested surface on the F. graminearum grown mycelia. The border area of colonies of $G$. roseum wild-type changed over days and pigment colors transformed from light-yellow to dark-orange, further evidence for the massive enzymatic activity between ZEN producer and its competitor. On the other hand, the Zes2 mutant colony appeared on the over-grown mycelia of $F$. graminearum but remained compact and the pigments scheme remained unchanged. One important reason could be that $\mathrm{G}$. roseum has a lactonase activity that is able to detoxify ZEN. We were unable to check the ZEN content in the plates with either G. roseum wild-type or Zes2 mutant co-incubated on F. graminearum. Our findings are supported with the results obtained by (Utermark and Karlovsky, 2007) where they observed partial inhibition of Zes2 mutants on ZEN concentration at $20 \mu \mathrm{g} / \mathrm{ml}$ on Czapek-Dox medium. Moreover, ZEN is an agent of interference, competition and it aids Fusarium species to protect the substrate against its competitors (Utermark and Karlovsky, 2007). ZEN may also play a role in fungal survival under adverse conditions (Kim et al., 2005). No significant differences were recorded in mycoparasite-pathogen inhibition bioassay. The inverted discs placement of $\mathrm{G}$. roseum wild-type and Zes2 mutant opposite to $\mathrm{F}$. 
graminearum on PDA did not display any significant differences. F. graminearum reached the opposite side of the PDA-plate quickly whereas G. roseum grew quite slowly. One explanation for the lack of differences could be the effect of medium. PDA is generally recommended for large production of mycelia. However, rice and corn are well-documented as good substrates for ZEN production (Greenhalgh et al., 1983). The abundance of $F$. graminearum in the natural environment showed that it survives and grows in very rough conditions as saprophytes and necrophytes with a diverse host range. However, G. roseum is non-pathogenic and abundance is shown in close proximity of Fusarium species (Knudsen et al., 1995a; J ensen et al., 2000)

It is evident that $G$. roseum is one of the most promising mycoparasite that resides in the proximity to toxigenic Fusarium species. Vakili (1992) showed that G. roseum reduced the frequency of Fusarium moniliforme in corn and decreased root colonization by the pathogen in field conditions. He suggested that G. roseum may be capable to suppress the growth of seed-transmitted pathogens. Knudsen (1994) observed the great competence of the mycoparasite against B. sorokinianainfected seedlings in sand and isolated the endophyte from distal and proximal root segments. Burgess et al. (1997) tested the effect of the mycoparasite against Botrytis cinerea on chickpea and found potential antagonistic effects and disease suppression. Xue (2003) investigated the impact of G. roseum on Clonostachys rosea on field pea root rot disease and recorded vast disease suppression. The rhizosphere colonization by the mycoparasite $G$. roseum against $F$. culmorum is documented in a few studies (Knudsen et al., 1995b; J ensen et al., 2000). Our investigations focus on the ecological effects of ZEN detoxification with $\mathrm{G}$. roseum and its implications on its producer. We used 5-pre-screened aggressive strains of F. graminearum that were earlier tested on both maize and wheat root infection. We recorded a similar amount of disease intensity on maize and wheat among 5pre-screened F. graminearum, whereas the disease severity was greater in maize than in wheat and so was the rate of emergence. We observed major differences between $\mathrm{G}$. roseum wild-type and Zes2 mutant co-inoculated plants with different F. graminearum strains. In order to reduce the probability, we used different chemotypes of F. graminearum co-inoculated with either $G$. roseum wild-type or Zes2 mutant. The emergence rate was much better in the $G$. roseum wild-type as compared to Zes2 mutant in maize. The diseased area marked on the disease scale represented a greater degree for the Zes2 mutant inoculated with the pathogen 
than the wild-type. Moreover, a large amount of biomass accumulated in root and shoot indexes of maize seedlings treated with G. roseum wild-type in maize. Our results also showcase a consistent trend in wheat seedlings, too. Most of the seedlings showed a sicker phenotype after co-inoculation with the Zes2 mutant than with the wild-type. The reduced germination and inhibition of seeds before emergence could be one of the initial effects of the pathogen to establish in and around the rhizosphere to compete for the nutrients. We achieved maximum infection rates in both maize and wheat in repeated experiments which is one of the important reasons of evaluating the differences between $G$. roseum wild-type and Zes2 mutant. Most of the seeds were exposed to the major outburst of the initial infection from the pathogen and fell prey to greater magnitude of blights and rots. (Malalase et al., 1973) and (Knudsen et al., 1995) evidenced that F. culmorum seed-infection takes place approximately 7 days after sowing in barley and wheat. The $G$. roseum wild-type treated seedlings of either maize or wheat resulted in greater yield in shoot and root indexes. However, heavy losses were only recorded in plants infected with F. graminearum and co-inoculated with Zes2 mutant. Knudsen and co-workers (1995) reported an increased germination rate in barley and an increased amount of seedlings biomass after the treatment with $G$. roseum. The pre- and post-soil treatment with G. roseum did not turn out to be useful. The soil treated before planting the seeds did not show any effects in disease suppression (data not shown). One important reason could be the average soil temperature or soil pH which is believed to play a central role in rhizosphere colonization.

ZEN is widely known as mycooestrogen rather than true mycotoxins (Hagler et al., 2001). It may play a role in fungal survival under adverse conditions or during grain colonization (Gaffoor et al., 2005; Kim et al., 2005). Its role has been investigated in some gene disruption studies. Gaffoor and co-workers (2005) studied the role of ZEN on wheat head infection and observed that ZEN wild-type and disrupted mutants showed the same amount of disease during infection. On the other hand, gene expression studies revealed that ZEN expression was increased during grain colonization, that it may aid the fungus to protect the substrate (Gaffoor et al., 2005). ZEN late production in maize fields or in stored grains are widely documented (Sutton, 1982; Martins and Martins, 2002).

To conclude so far, ZEN is not a virulence factor for $F$. graminearum this is explained with several functional genetic studies (Gaffoor et al., 2005; Kim et al., 
2005; Gaffoor and Trail, 2006; Lysoe et al., 2006). Its expression during the grain colonization further supports our hypothesis that ZEN is potentially involved to establish the fungus in grain and protect the resource against its competitors. It is suggested that $G$. roseum potentially reduces the $F$. graminearum colonization of the seeds and in rhizosphere. The Zes2 mutant of G. roseum was found to be more sensitive to ZEN, as it was more affected and seedlings were less protected in comparison to $\mathrm{G}$. roseum wild-type.

\section{References}

1. Brandfass, C. and P. Karlovsky. 2008. Upscaled CTAB-based DNA extraction and real-time PCR assays for Fusarium culmorum and F. graminearum DNA in plant material with reduced sampling error. International Journal of Molecular Sciences 9:2306-2321.

2. Burgess, D. R., T. Bretag, and P. J. Keane. 1997. Biocontrol of seedborne Botrytis cinerea in chickpea with Gliocladium roseum. Plant Pathology 46:298-305.

3. Desjardins, A. E. and R. H. Proctor. 2001. Biochemistry and genetics of Fusarium toxins. In B.A.Summerell, J.F.Leslie, D. Backhouse, W. L. Bryden, and L.W. Burgess (ed.), Fusarium. Paul E. Nelson Memorial Symposium50-69.

4. Elsharkawy, S. and Y. J. Abulhajj. 1988. Microbial cleavage of zearalenone. Xenobiotica 18:365-371.

5. Gaffoor, I., D. W. Brown, R. Plattner, R. H. Proctor, W. H. Qi, and F. Trail. 2005. Functional analysis of the polyketide synthase genes in the filamentous fungus Gibberella zeae (Anamorph Fusarium graminearum). Eukaryotic Cell 4: 1926-1933.

6. Gaffoor, I. and F. Trail. 2006. Characterization of two polyketide synthase genes involved in zearalenone biosynthesis in Gibberella zeae. Applied and Environmental Microbiology 72:1793-1799.

7. Goswami, R. S. and H. C. Kistler. 2004. Heading for disaster: Fusarium graminearum on cereal crops. Mol. Plant Pathol. 5:515-525.

8. Greenhalgh, R., G. A. Neish, and J. D. Miller. 1983. Deoxynivalenol, acetyl deoxynivalenol, and zearalenone formation by canadian isolates of Fusarium graminearum on solid substrates. Applied and Environmental Microbiology 46:625-629.

9. Hagler, W. M., N. R. Towers, C. J. Mirocha, R. M. Eppley, and W. M. Bryden. 2001. Zearalenone: mycotoxin or mycoestrogen ? In B.A.Summerell, J.F.Leslie, D.Backhouse, W.L.Bryden, and L.W.Burgess (ed.), Fusarium.Paul E. Nelson Memorial Symposium321-331. 
10. Jensen, B., I. M. B. Knudsen, and D. F. Jensen. 2000. Biological seed treatment of cereals with fresh and long-term stored formulations of Clonostachys rosea: Biocontrol efficacy against Fusarium culmorum. European J ournal of Plant Pathology 106:233-242.

11. Karlovsky, P. 1994. Inhibition of imidazoleglycerolphosphate dehydratase of Phytophthora parasitica by aminotriazole in situ and after cloning and expression of the respective gene (His3) in Escherichia coli. Journal of Phytopathology-Phytopathologische Zeitschrift 141:121-126.

12. Karlovsky, P. 1999. Biological detoxification of fungal toxins and its use in plant breeding, feed and food production. Natural Toxins 7:1-23.

13. Kim, Y. T., Y. R. Lee, J. M. J in, K. H. Han, H. Kim, J. C. Kim, T. Lee, S. H. Yun, and Y. W. Lee. 2005. Two different polyketide synthase genes are required for synthesis of zearalenone in Gibberella zeae. Molecular Microbiology 58:1102-1113.

14. Knudsen, I. M. B. 1994. Biological control of seed borne diseases. PhD thesis. $\mathrm{PhD}$ thesis. Frederiksberg: The Royal Veterinary and Agricultural University.

15. Knudsen, I. M. B., J. Hockenhull, and D. F. Jensen. 1995a. Biocontrol of seedling diseases of barley and wheat caused by Fusarium culmorum and Bipolaris sorokiniana - Effects of selected fungal antagonists on growth and yield components. Plant Pathology 44:467-477.

16. Knudsen, I. M. B., J. Hockenhull, and D. F. Jensen. 1995b. Biocontrol of seedling diseases of barley and wheat caused by Fusarium culmorum and Bipolaris sorokiniana - Effects of selected fungal antagonists on growth and yield components. Plant Pathology 44:467-477.

17. Logrieco, A., G. Mule, A. Moretti, and A. Bottalico. 2002. Toxigenic Fusarium species and mycotoxins associated with maize ear rot in Europe. European J ournal of Plant Pathology 108:597-609.

18. Lysoe, E., S. S. Klemsdal, K. R. Bone, R. J. N. Frandsen, T. J ohansen, U. Thrane, and $\mathrm{H}$. Giese. 2006. The PKS4 gene of Fusarium graminearum is essential for zearalenone production. Applied and Environmental Microbiology 72: 3924-3932.

19. Malalase, R., Sanderso, F., and J. Colhoun. 1973. Fusarium diseases of cereals - Penetration and invasion of wheat seedlings by Fusarium culmorum and Fusarium nivale. Transactions of the British Mycological Society 60:453-.

20. Martins, M. L. and H. M. Martins. 2002. Influence of water activity, temperature and incubation time on the simultaneous production of deoxynivalenol and zearalenone in corn (Zea mays) by Fusarium graminearum. Food Chemistry 79:315-318.

21. Munkvold, G. P. 2003. Epidemiology of Fusarium diseases and their mycotoxins in maize ears. European J ournal of Plant Pathology 109:705-713.

22. Nicholson, P., D. R. Simpson, G. Weston, H. N. Rezanoor, A. K. Lees, D. W. Parry, and D. Joyce. 1998. Detection and quantification of Fusarium 
culmorum and Fusarium graminearum in cereals using PCR assays. Physiological and Molecular Plant Pathology 53:17-37.

23. Papavizas, G. C. 1985. Trichoderma and Gliocladium - Biology, ecology, and potential for biocontrol. Annual Review of Phytopathology 23:23-54.

24. Parry, D. W., P. J enkinson, and L. Mcleod. 1995. Fusarium Ear Blight (Scab) in small grain cereals - A Review. Plant Pathology 44:207-238.

25. Shier, W. T., A. C. Shier, W. Xie, and C. J. Mirocha. 2001. Structure activity relationships for human estrogenic activity in zearalenone mycotoxins. Toxicon 39: 1435-1438.

26. Sutton, J. C. 1982. Epidemiology of wheat head blight and maize ear rot caused by Fusarium graminearum. Canadian Journal of Plant Pathology 4: 195.

27. Takahashi-Ando, N., M. Kimura, H. Kakeya, H. Osada, and I. Yamaguchi. 2002. A novel lactonohydrolase responsible for the detoxification of zearalenone: enzyme purification and gene cloning. Biochem.J. 365: 1-6.

28. Utermark, J. and P. Karlovsky. 2007. Role of zearalenone lactonase in protection of Gliocladium roseum from fungitoxic effects of the mycotoxin zearalenone. Applied and Environmental Microbiology 73:637-642.

29. Vakili, N. G. 1992. Biological seed treatment of corn with mycopathogenic fungi. Journal of Phytopathology-Phytopathologische Zeitschrift 134:313323.

30. Vidnes, A., B. Paulsen, and C. Bergsten. 2003. Report from SCOOP task3.2.10 "Collection of occurrence data of Fusarium toxins in food and assessment of dietary intake by the population of EU member states"-Subtask II: zearalenone. [Online.]

http:/ / europa. eu.int/comm/ food/ fs/ scoop/ task3210. pdf.

31. Xue, A. G. 2003. Biological control of pathogens causing root rot complex in field pea using Clonostachys rosea strain ACM941. Phytopathology 93:329335. 


\section{Chapter 3: Pathogenicity and mycotoxin production of different F.graminearum strains on maize and wheat ear and root infection}

Awais Ahmed, Katharina Döll and Petr Karlovsky

Molecular Phytopathology \& Mycotoxin Research Unit, Georg-August University, Goettingen, Germany.

\section{Abstract}

F. graminearum is an important pathogen that causes head blight and root rots in wheat and ear and root rots in maize. Trichothecences type B deoxynivalenol (DON) its acetylated derivatives, nivalenol (NIV) and zearalenone (ZEN) are common mycotoxins produced by the fungus. DON occurrence is high in cereals while NIV and ZEN are potential contaminants in maize. We investigated $22 \mathrm{~F}$. graminearum strains on wheat-maize-ear-root infection system. The greatest disease severity was observed on wheat heads; however increased root infection took place in maize. The strains virulence was positively correlated among wheat heads/maize ears and root infections of wheat and maize. The DON content was also positively correlated with disease severity in both wheat and maize ears. However, no positive correlation was found between DON contents and fungal DNA in maize because of the different chemotypes. In wheat, a tight correlation was found between DON and DNA content. NIV was detected in lower amounts than DON while all maize ears showed lower ZEN accumulation. In our study some of the strains produced 15ADON on maize which cause for great concern. The amount of mycotoxin was a decisive factor in pathogen virulence in both wheat and maize ears. We may conclude that wheat heads are more susceptible than roots whilst in maize both ears and roots are susceptible to Fusarium infection. 


\section{Introduction}

F. graminearum Schwabe (teleomorph Gibberella zeae [Schwein] Petch) is the most associated pathogen to head blight (FHB) and root rot in wheat (Triticum aestivum) as well as ear and root rot infection in maize (Zea mays) (Logrieco et al., 2002; Munkvold, 2003; Parry et al., 1995). Maize residues serve as a potential reservoir for pathogen, where it survives as mycelia and disperse to an appropriate host or substrate during the vegetation period (Gilbert and Fernando, 2004; Sutton, 1982). F. graminearum is as pathogenic in maize as it is in wheat, where it is the second most important host. The FHB symptoms on wheat appeared as blighted spikes and purple-black perithecia or pink sporodochia which are present on glumes. Infected maize ears are partially covered with pink- to reddish-colored mold on kernels, serving as a large sink for mycotoxins accumulation (Goswami and Kistler, 2004; Munkvold, 2003).

Being a multifaceted pathogen, it produces large quantities of mycotoxins. The trichothecene type B deoxynivalenol (DON) and its acetylated derivatives 3acetyldeoxynivalenol (3ADON), and 15-acetyldeoxynivalenol (15ADON) and nivalenol (NIV) as well as the estrogenic polyketide zearalenone (ZEN) are frequently encountered in cereals and maize (Desjardins and Hohn, 1997). DON is a major contaminant in wheat with its acetylated derivatives (3ADON or 15ADON) present in lower concentrations. 3ADON or 15ADON are less toxic to mammals than DON (Kimura et al., 1998), but may be equally toxic to plants (Eudes et al., 2000). However, NIV may be the predominant mycotoxin in maize which is ten-fold more toxic than DON or its acetylated derivatives (Ryu et al., 1988; Snij ders, 1990). The mycotoxins contamination is a great risk to humans and animals and causes substantial fiscal deficits in economic production (Nganje et al., 2004; Snijders, 1990).

The targeted role of trichothecenes in $\mathrm{F}$. graminearum aggressiveness has been tested in several gene-knock-out studies. In wheat heads inoculated with F. graminearum DON disrupted (Tri5-) mutant, resulted in initial infection at point of inoculation but was not able to spread beyond the whole spike unlike the wild-type (Tri5+) (Bai et al., 2002). Similar results were obtained in maize field trials in which Harris et al. (1999) tested DON (Tri5-) mutants and reported them less virulent in comparison to wild-type (Tri5t) in Gibberella ear rot. The role of NIV 
remains a topic of great speculation. Maier et al. (2006) reported NIV production has little or no effect on wheat ears, but it appeared to be a virulence factor in maize ear rot. In wheat, barley and triticale seedling infection, Wang et al. reported that DON (Tri5-) mutants were less virulent than DON producers wildtype (Tri5+) with some inconsistencies (Wang et al., 2006). Nevertheless, a large genetic variation exists within the Fusarium species complex which shifts the virulence, toxin production and host interaction in response to environmental fluctuations (Desjardins, 2006). So far two types of resistance mechanisms are well recognized to FHB: resistance to the initial infection (type I resistance) and resistance to spread within the head (type II resistance) (Schroeder and Christensen, 1963). The prevalence of DON in cereals and NIV in maize suggests that both chemotypes possess selective advantages on different host species and operate in different phases of the pathogen life cycles (Ward et al., 2002). The ability to produce DON and NIV co-exists within species and chemotype represent in individual strain, but their proportions highly fluctuate with seasonal rotations (J ennings et al., 2004). The variability in damage caused by F.graminearum range in either total destruction of the host or minor damage. Therefore, the hostpathogen interaction is characterized as non-race and non-host specific (Vaneeuwijk et al., 1995). It is suggested that in United States, different F. graminearum 'chemotypes' are replacing the other species which indicate an influx of unique genetic populations (Gale et al., 2007). Many reports from Europe indicate that F. graminearum is predominating other species such as F.culmorum (Xu et al., 2005), possibly due to brief climatic fluctuations and ecological differences concerning aggressiveness and pathogenicity of the different species.

Numbers of studies were conducted in order to understand the role of $F$. graminearum in host-pathogen interactions on different hosts. Carter et al. (2002) studied the genetic variation and pathogenicity in reference to the chemotype among different $F$. graminearum strains on maize and wheat after seedling infection. Goswami and Kistler (2005) tested different F. graminearum strains and other species on wheat and rice after head infection. Further more, Reid et al. (1996) monitored the DON distribution by comparing maize hybrids inoculated with F. graminearum. However, Miedaner et al. (2010) compared different F. graminearum and $F$. verticillioides strains on susceptible and resistant maize inbred lines. 
There is not much data available in comparison of different $F$. graminearum chemotypes on maize and wheat after ear and root infection. Understanding this multi-fold pathogen, we have tested pathogenicity and mycotoxins accumulation during infection with different $F$. graminearum strains. The objective of our study was (1) to evaluate the aggressiveness of twenty two different F.graminearum strains on maize and wheat ear and root infection and (2) to quantify the trichothecenes accumulation and fungal biomass in infected samples.

\section{Material and Methods}

Table 1. Assigned code, host, original code and source of different Fusarium graminearum strains.

\begin{tabular}{llll}
\hline Assigned code & Chemotype & Code & Source \\
\hline F1 & DON +3ADON & Fg 210 & University Goettingen, Germany \\
F3 & DON + 3ADON & Fg 102.3 & University Goettingen, Germany \\
F4 & NIV & Fg 71 & University Hohenheim, Germany \\
F7 & DON + 3ADON & Fg 7-28 & No. 893, DSMZ, Germany (maize) \\
F9 & DON + 3ADON & Fg 210.1 & University Goettingen, Germany \\
F10 & DON + 3ADON & Fg 201.2 & University Goettingen, Germany \\
F11 & DON + 3ADON & Fg 203.1 & University Goettingen, Germany \\
F16 & DON + 15ADON & Fg 8-3 & University Hohenheim, Germany \\
F17 & DON + 3ADON & Fg 7-45 & No. 316.73, CBS, Netherlands \\
F18 & NIV & Fg 10-3 & University Hohenheim, Germany \\
F20 & DON + 3ADON & Fg 144 & University Goettingen, Germany \\
F21 & NIV & Fg 108.1 & University Goettingen, Germany \\
F26 & DON + 15ADON & Fg 12 & University Goettingen, Germany (rye) \\
F27 & DON + 3ADON & Fg 211.1 & University Goettingen, Germany \\
F30 & DON + 3ADON & Fg 106.2 & University Goettingen, Germany \\
F31 & DON + 3ADON & Fg 201.1 & University Goettingen, Germany \\
F32 & DON + 3ADON & Fg 202.3 & University Goettingen, Germany \\
F38 & DON + 3ADON & Fg 7-34 & Sydney University, Australia (wheat crown rot) \\
F40 & DON + 3ADON & Fg 104.2 & University Goettingen, Germany \\
F42 & NIV & Fg 7-2 & No. 414.86, CBS, Netherlands (banana) \\
F46 & DON + 3ADON & Fg 108.2 & University Goettingen, Germany \\
F47 & DON + 3ADON & Fg 104.1 & University Goettingen, Germany \\
\hline
\end{tabular}

\section{Fungal strains, culture conditions and media}

Considering the broad host range of F.graminearum, we included 22 strains with different hosts and different geographical background in this study which are listed in (Table 1). Fungal strains were maintained as spore suspension with $15 \%$ glycerol with sterile tap water and frozen at $-80^{\circ} \mathrm{C}$ until use. Fungal inoculum was produced 
on mung bean liquid medium slightly modified from (Bai and Shaner, 1996) by injecting $50 \mu \mathrm{l}$ of spore suspension in an Erlenmeyer flask with $100 \mathrm{ml}$ of medium for four days at $28^{\circ} \mathrm{C}$ and shaking at $200 \mathrm{rpm}$. Spores were filtered through a cotton ball placed directly into funnel at the top of Erlenmeyer flask and the filtrate was transferred to a $50 \mathrm{ml}$ reaction tube. The filtrate was centrifuged for 10 minutes at $4500 \mathrm{rpm}$; the supernatants were removed carefully. Then the pellets were re-suspended in sterile tap water. Fungal spores count determination was done by placing few microliter of spore suspension into a Thoma counting chamber. The final concentration was adjusted according to the experimental inoculation scheme.

\section{Maize and wheat cultivars and growth conditions}

The moderately susceptible cultivars for root infection, maize cv. 'KABANAS' and wheat cV. 'CUBUS' were obtained from KWS SAAT AG, Einbeck, Germany and KWS Lochow $\mathrm{GmbH}$, Bergen, Germany respectively. The highly susceptible wheat cultivar to FHB, Apogee Triticum aestivum 'USU-Apogee' was obtained from federal government institute Darmstadt, Germany and originally published by (Bugbee et al., 1997). For wheat head infection $4 \mathrm{cv}$. Apogee seeds were sown in $9 \mathrm{~cm} \varnothing$ plastic pots, filled with the mixture of flower substrate + sand (2:1). After two weeks of plant growth, seedlings were thinned to two plants to each pot. All cultivars either for root infection or head inoculation were grown in a greenhouse at approximately $24 \pm 3^{\circ} \mathrm{C}$ with $16 \mathrm{~h}$ supplemental light (400W Philips bulbs with PIA technology) and with the relative humidity between 40-60\% Seedlings were watered daily and fertilized in week-intervals with NPK (15-11-15).

\section{Aggressiveness test on maize and wheat root}

Each seed was planted in mixture of plant substrate + sand ratio $(2: 1)$ in $4.5 \times 5 \mathrm{~cm}$ $\varnothing$ plastic pots. We used the seed infection method for pathogenicity modified slightly from (Knudsen et al., 1995). To facilitate the infection, we placed a piece of filter paper under each seed. The seed was inoculated with the spore suspension $\left(1.5^{*} 10^{5} / \mathrm{ml}\right)$. Each treatment was designated from F1 to F47 (Numeric with (F) letter represent different F. graminearum strains in graph-legend) or mock control (water 
only). The codes are denoted in (Table 1). Ten replicates were used for each treatment and the experiment was repeated twice. The plants were harvested 28 days after inoculation (dpi). The disease index (DI) was scored at harvest, using the disease scale DI described by (Knudsen et al., 1995) with slight modification where 0 - no disease symptoms; 1 - brown pin point lesions on the coleoptile and/ or roots; 2 - extended browning of the coleoptile and/ or browning/reddening of the roots; 3 - dead coleoptile and/or extended browning/reddening of the roots; and 4 browning extending beyond the coleoptile on the above-ground plant parts or dead plant or ungerminated kernel. The percentage of emerged seedlings was calculated after one week and the final seedling stand was evaluated at harvest. Shoot and root biomass indexes were measured after harvest.

\section{Aggressiveness test on wheat heads}

The macroconidia collected from Mung bean medium adjusted to $1 * 10^{5} / \mathrm{ml}$ by adding few $\mu \mathrm{l}$ of spore suspension into Thoma counting chamber. Additionally, a surface-active agent (Silwet Gold) was added to a final concentration of $0.01 \%$ to the inoculum for a better adherence to the host tissue. The point inoculation method (modified from Maier et al., 2006) was used to inject the spores in the middle of the floret, using insulin syringes at early to mid- anthesis stage on day 36. Each wheat head was individually inoculated with the 2000 conidia/ 20ul. After inoculation, florets were covered with small plastic bags for $\sim 48$ hours to retain the optimum moisture conditions for infection. Each treatment consisted of a total of 20 plants with two plants within a pot and ten pots per treatment. The heads inoculated with sterile water (containing $0.01 \%$ Silwet Gold) were used as negative (mock-inoculated) controls. Head blight symptoms were rated at $7 \mathrm{dpi}$ by counting the number of spikelets (1-100\%) showing disease symptoms (necrosis and/or bleaching of palea/lemma) as described by (Stack and McMullan, 1995). Three additional ratings were carried out in order to determine the disease progression until harvest. Several other parameters were also evaluated, such as number of grains per spike, grain and spike weight etc. After harvest the infected spikes were transferred to paper bags and stored at 40 degree centigrade in oven for a week. Individual spikes were threshed (Small domestic thresher type, Linca Scientific Instrument, Tel-Aviv, Israel) and weighd. Grains were counted (Numigral Seed 
Counter, Durant, Soild Star 1800) and subsequently milled with a cross hammer mill (Retsch, Haan, Germany) in order to determine DNA and mycotoxins contents.

\section{Maize ears trial design, infection method and disease rating}

The field trial was conducted from August till September in 2009, as a complete randomized block design in a designated trial area near Goettingen Germany. The treatments were split into four small groups, where each group contained five replicates and total of 20 replicates for the whole treatment. Two negative controls were used as standards for background infection. Water inoculated cobs (water injected cobs) and healthy cobs, which were without inoculation.

The silk channel inoculation method was used for cob infection, as described by (Reid et al. 1996) with slight modification. Individual plants were inoculated at day five of silk emergence by injecting $105 / \mathrm{ml}$ of spore suspension into the silk channel of the primary ear using a graduated; 2-ml self-filled automated vaccinator (type Ultra-Asept).

At physiological maturity, cobs were rated for disease severity based on a percentage scale published in (Reid and Hamilton, 1996). Additionally, fresh and dry biomass was weighed individually for each cob. After oven drying at 50 degree centigrade for seven days, grains were manually removed and ground with a cross hammer mill (Retsch 2M 200, Retsch, Germany) at $1 \mathrm{~mm}$ sieve diameter. Ten samples were taken for further mycotoxins analysis with HPLC-MS/MS and DNA quantification with Real Time PCR.

\section{DNA extraction of wheat and maize flour (CTAB method)}

Due to heavy infestation of wheat grains, of each treatment 20 samples were pooled into 5 collective replicates that means 1 replicate is a pooled sample of 4 individual replicates. DNA was extracted with a CTAB method published by Brandfass and Karlovsky (2008), modified as follows: $50 \mathrm{mg}$ of flour was blended into $1 \mathrm{ml} \mathrm{CTAB}$-buffer. The DNA was dissolved in $50 \mu \mathrm{l} \mathrm{TE}$. The same DNA extraction procedure was followed for maize flour. The $500 \mathrm{mg}$ of maize flour was extracted with $8 \mathrm{ml} \mathrm{CTAB-buffer,} \mathrm{and} \mathrm{total} \mathrm{DNA} \mathrm{was} \mathrm{re-suspended} \mathrm{in} 100 \mu \mathrm{l} \mathrm{TE}$-buffer. The 
DNA quantity was checked by agarose gel electrophoresis before DNA quantification with Real Time PCR. A 1:10 dilution was used in the Real-time PCR analysis.

\section{F. graminearum DNA quantification by Real Time PCR}

Real-time PCR protocol was developed by Brandfass \& Karlovsky (2008) based on specific primers to F. graminearum described by (Nicholson et al., 1998). The PCR amplification was done according to cited work (Brandfass and Karlovsky, 2008), modified as follows: Reaction mixture consisted of $1 \times$ ABsolute Blue QPCR SYBR Green Fluorescein Mix (containing Thermo Start TM DNA-Polymerase, $3 \mathrm{mM} \mathrm{MgCl2,}$ dNTP Mixture, SYBR Green I, and $10 \mathrm{nM}$ Fluorescein, Abgene Limited, United Kingdom), and the initial denaturation was extended for $13.5 \mathrm{~min}$ as required for the activation of the polymerase.. The fluorescence was measured after each PCR step on iCycler (BioRad, Hercules, CA, USA). Calibration curve was carried out with purified $\mathrm{F}$. graminearum DNA in $0.5 \mathrm{pg}, 5 \mathrm{pg}, 50 \mathrm{pg}$ and $500 \mathrm{pg}$ added to clean flour matrix either wheat or maize. PCR product ratios were converted to DNA concentrations (picograms of total DNA), then to microgram per kilogram $(\mu \mathrm{g} / \mathrm{kg})$ flour material.

\section{Mycotoxin extraction from wheat flour}

A portion of $300 \mathrm{mg}$ of wheat flour was extracted with $5 \mathrm{ml}$ of $84 \%$ acetonitrile with double distilled water in a $15 \mathrm{ml}$ sealed falcon tube, shaken at $150 \mathrm{rpm}$ for ca. 18 hours. Then the samples were centrifuged for 6 minutes at $4500 \mathrm{rpm}$. One $\mathrm{ml}$ of supernatant was taken from the upper phase of the falcon, transferred into $2 \mathrm{ml}$ reaction tubes and dried in a vacuum concentrator (Martin Christ, Osterode, Germany) over night. $1 \mathrm{ml}$ of methanol $50 \%$ was added to the evaporated sample and put into an ultrasonic bath for a few seconds until it was completely dissolved. After mixing briefly $400 \mu \mathrm{l}$ hexane was added and mixed again. The sample was centrifuged again at maximum rpm and lower phase was transferred carefully into a new reaction tube. All samples were diluted 1:60 prior to mycotoxin analysis. For HPLC-MS/ MS analysis $600 \mu$ were transferred into vials. 


\section{Mycotoxin extraction maize flour}

Maize flour $4 \mathrm{~g}$ was extracted overnight with $40 \mathrm{ml}$ of methanol/ isopropanol/ water 80:5:15. The sample was centrifuged at $4500 \mathrm{rpm}$ for $10 \mathrm{~min}$, and one millilitre of supernatant was transferred to a $2 \mathrm{ml}$ reaction tube, evaporated until dry in a vacuum concentrator (Martin Christ, Osterode, Germany), redissolved in $1 \mathrm{ml}$ methanol/water $50 \%$ and defatted using $500 \mu$ of hexane. The sample was centrifuged again at maximum rpm and the lower phase was taken up carefully and transferred into new a $1.5 \mathrm{ml}$ - tube. All samples were diluted 1:40 prior to mycotoxin analysis. For HPLC-MS/ MS analysis $600 \mu$ were transferred into vials.

\section{Mycotoxin analysis (HPLC-MS/MS)}

The wheat head infected samples were analyzed for DON, NIV and ZEN, therefore maize cobs were quantified for DON, 3DON, 15ADON, NIV and ZEN with HPLCMS/MS. The HPLC-MS/MS system used for determination of the mycotoxins listed above consisted of ProStar 210 pumps (Varian, Darmstadt, Germany), column oven Mistral 510 (Varian, Darmstadt, Germany), separating column Kinetex C18 (50x2, $1 \mathrm{~mm}, 2.6 \mu \mathrm{m})$ (Phenomenex, Aschaffenburg, Germany) and 1200 LC Triple Quadrupole mass spectrometer with electrospray ionisation-interface (Varian, Darmstadt). Standards were prepared with spiked blank samples, which were extracted as described above.

\section{Statistical analysis}

Statistical analysis was carried out with the software package "Sigma plot" (Systat Software, Inc. USA). The data were analyzed by Kruskal-Wallis One Way Analysis of Variance on Ranks ( $P \varangle 0.05$ ) and pairwise multiple comparison vs. Control was done by (Dunn's Method) $(P \varangle 0.05)$. The Pearson Product Moment Correlation was used to test correlations different correlations in wheat heads, maize ears and wheat and maize root infection at significant level $(P \varangle 0.05)$. 


\section{Results}

\section{F. graminearum aggressiveness on maize and wheat root}

In maize root inoculation, most of the seeds received severe infection and resulted in poor germination. The final seedling stands in different F. graminearum treatments remained between 30-100\% However, the disease severity was relatively lower in wheat seedling infection where the number of seedlings surviving at harvest remained 60-100 \% compared to the water control (Fig.1). The highest mortality ratio in maize seedlings was recorded in F26 (70\%) and the mortality trend remained consistent among F11, F4 and F10 up to (50\%). However, in wheat seedlings the greatest mortality ratio up to $80 \%$ was recorded in $\mathrm{F} 47$ infected plants, (which is a DON producer) and constant mortality ratios among F26 and F11 up to (40\%). The seedling mortality was almost uniform on maize and wheat for the strains F10, F11, F26 and F47 (DON producers). However, F4 remained aggressive on both maize and wheat seedlings which is a potential NIV producer.

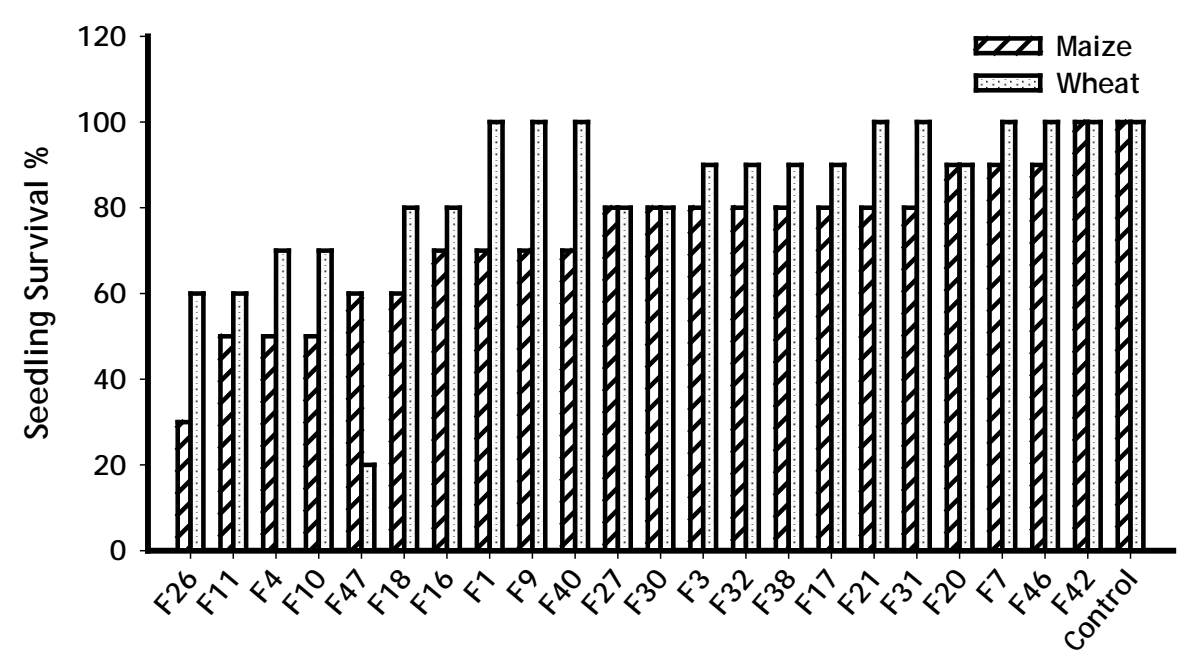

F.graminearum strains

Figure 1. The effect of different chemotypes strains of $F$. graminearum on maize and wheat seedling survival 4-week-long duration until harvest. Each bar is a pooled mean of 10 replicates per treatment. 
The disease evaluation on both maize and wheat posed significant differences. The disease index scored on maize ranged between $0.2-3.3$ on the scale, ( $0=$ healthy and $4=$ dead seeds). The disease index was slightly lower on wheat seedling where the disease was rated between $0.35-2.3$. The strain F42 which was rated 0.2 on maize and 0.45 on wheat (Fig.2). The virulence was very consistent with the strain F26 which exhibited elevated disease severity on both maize and wheat seedling. Most infected seeds were unable to germinate and yielded in very poor seedling stand at harvest. There were several other symptoms recorded throughout the pathogenicity test in greenhouse. The strong symptoms for disease evaluation such as severe inhibition of seeds after infection resulted in lower numbers of germinated seeds and severe deformation of the seedlings during the first week after inoculation. The deformed seedlings were later completely blighted and affected with severe root rot symptoms which retard all physiological processes, few seedlings survived until harvest but remained very malnourished and impaired in phenotype. The disease severity (virulence) among different F. graminearum strains on maize and wheat was positively correlated $(r=0.527)$ at the significant level at $P \varangle 0.05$. There were no further significant correlations found among maize and wheat.

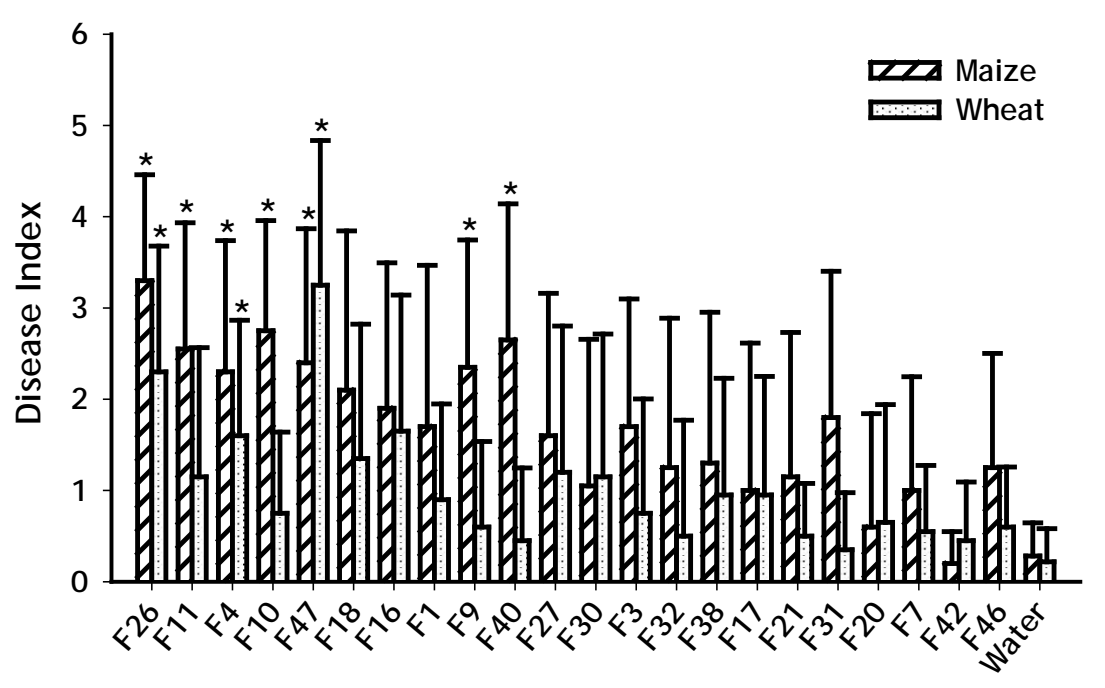

F.graminearum strains

Figure 2. The virulence of different chemotypes of $F$. graminearum on maize and wheat seedlings. For disease scale description see section material \& methods. Capped bars with the asterisk (*) are significantly different with multiple comparisons vs. Control (Dunn's Method) at $P=\varangle 0.05$ using Kruskal-Wallis One Way ANOVA on Ranks. Each capped-bar marks a pooled mean of 10 replicates per treatment with standard deviation. 
The largest reduction of seedling biomass index for maize after root inoculation was $49 \%$ for (F10). However, the lowest reduction of seedling biomass of infected roots was for (F17) which did not differ from the control, which was designated as $100 \%$ However, infected wheat root biomass was reduced up to $55 \%$ for (F4) and the lowest reduction $95 \%$ (F32) of the control (Fig.3). The infection resulted in poor yield of maize seedling shoot biomass. The lowest biomass index was measured in F10 infected seedlings, which was $42.6 \%$ and the highest limit was 100 $\%$ in (F30) compared to the non-inoculated (water control). The wheat seedlings also produced relatively poor shoot biomass. The lowest with $53 \%$ (F26) and the highest limit was $100 \%$ (F31) compared to the control (Fig.4).

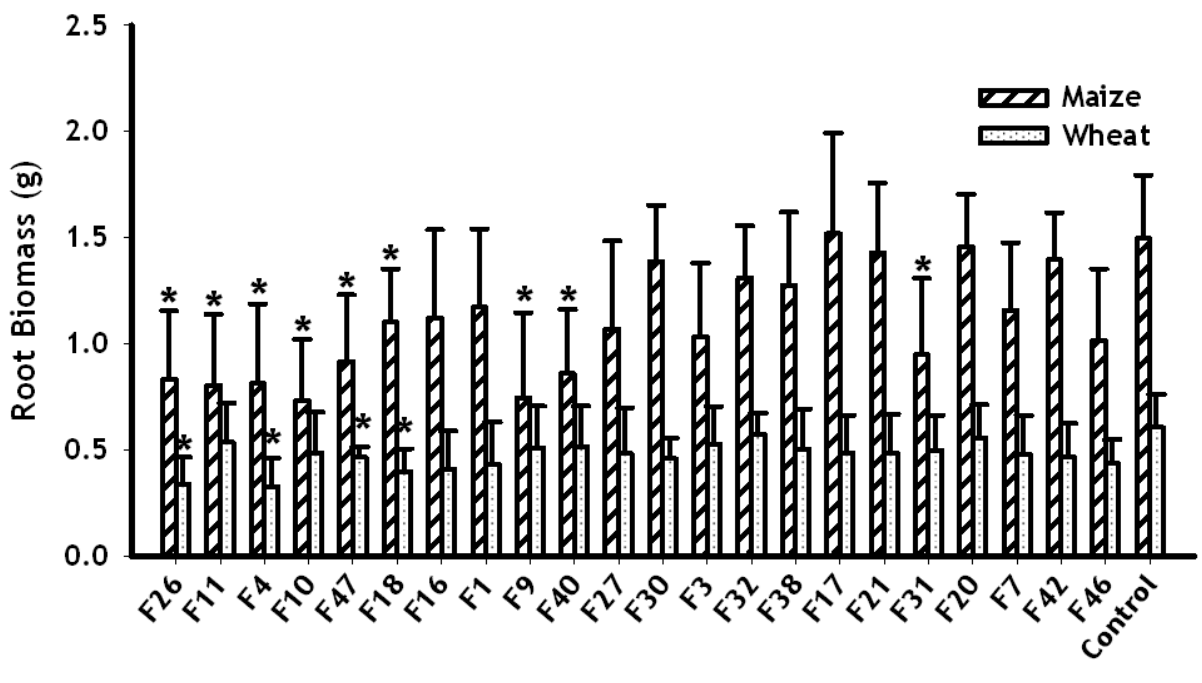

F.graminearum strains

Figure 3. The virulence effect of different chemotypes of $F$. graminearum on seedling root biomass on maize and wheat seedling. For disease scale description see section material \& methods. Capped bars with the asterisk $(*)$ are significantly different with multiple comparisons vs. Control (Dunn's Method) at $P=\varangle 0.05$ using Kruskal-Wallis One Way ANOVA on Ranks. Each capped-bar marks a pooled mean of 10 replicates per treatment with standard deviation. 


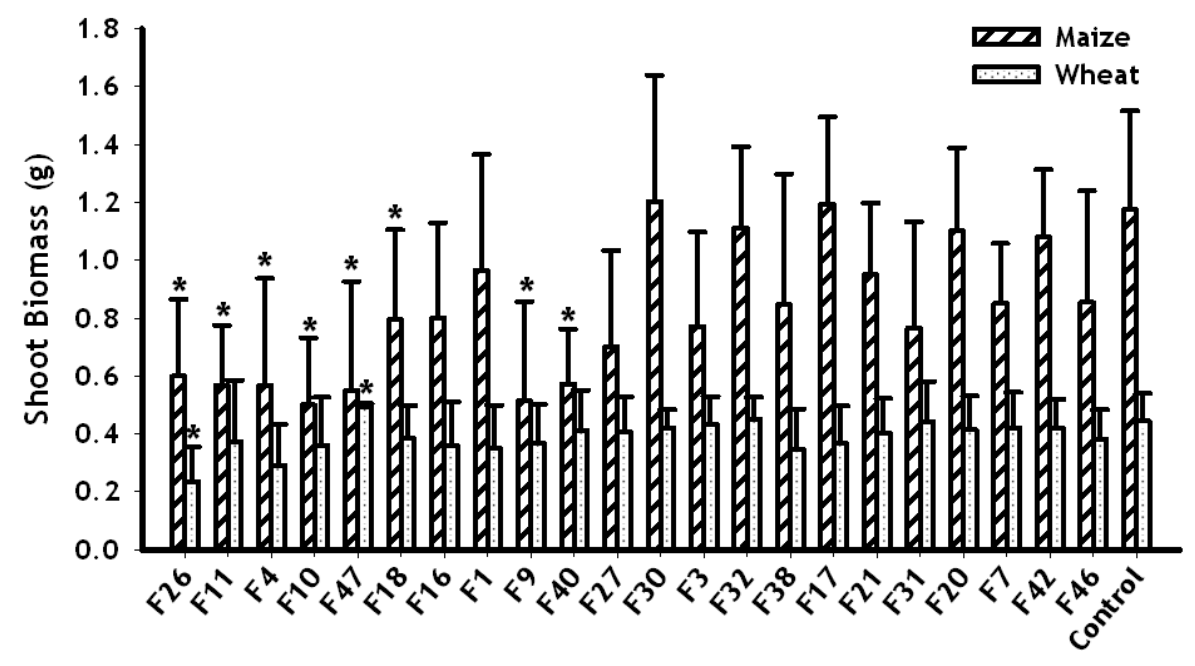

F.graminearum strains

Figure 4. The virulence effect of different chemotypes of $F$. graminearum on seedling shoot biomass on maize and wheat seedling. Disease scale details, (see section: Material \& Methods). Capped bars with the asterisk $(*)$ are significantly different with multiple comparisons vs. control (Dunn's Method) at $P \varangle 0.05$ ) using Kruskal-Wallis One Way ANOVA on Ranks. Each capped-bar marks a pooled mean of 10 replicates per treatment with standard deviation.

\section{F. graminearum aggressiveness on wheat heads}

Numerous F. graminearum strains were able to spread through wheat heads from the point of inoculation to other spikelets. Considerable virulence was recorded in all strains regardless of their chemotypes. The first disease symptoms appeared in 24 hours post inoculation. The disease rating was highly variable among isolates during the first week; it ranged between $4-22 \%$ in all strains. The first disease rating confirmed that all tested strains produced initial infection. The disease severity increase several fold during the second week post inoculation and ranged between $11-79 \%$ increasing to $14-100 \%$ in the third week of rating. The disease severity process slowed down close to harvest as it achieved maximum colonization on spike or nutrient depletion and recorded disease ratings between 16-100\% (Fig.5). At harvest, the severely diseased spikes looked completely bleached and blighted. The grain was shriveled and reduced in weight. The head blight symptoms were very visible which increased several fold after inoculation. Some strains F21, F40 and F42 were able to produce pathogenicity but were unable to spread to the entire spike. However, no particular pattern could be observed in distribution among highly aggressive to moderately aggressive strains. The fungus started 
spreading within the wheat heads and disease symptoms were observed in both directions. However, the pathogen spreading towards lower spikelets was more pronounced in less aggressive strains. The phenotypic variation in symptoms development on infected heads was recorded among the strains which produced different amounts of mycotoxins. Wheat heads inoculated with less aggressive strains appeared to show more necrosis in the form of blackish lesions on the infected spikelets rather than bleaching and deformation of awns which was commonly seen in plants infected with the highly aggressive strains.

(A)

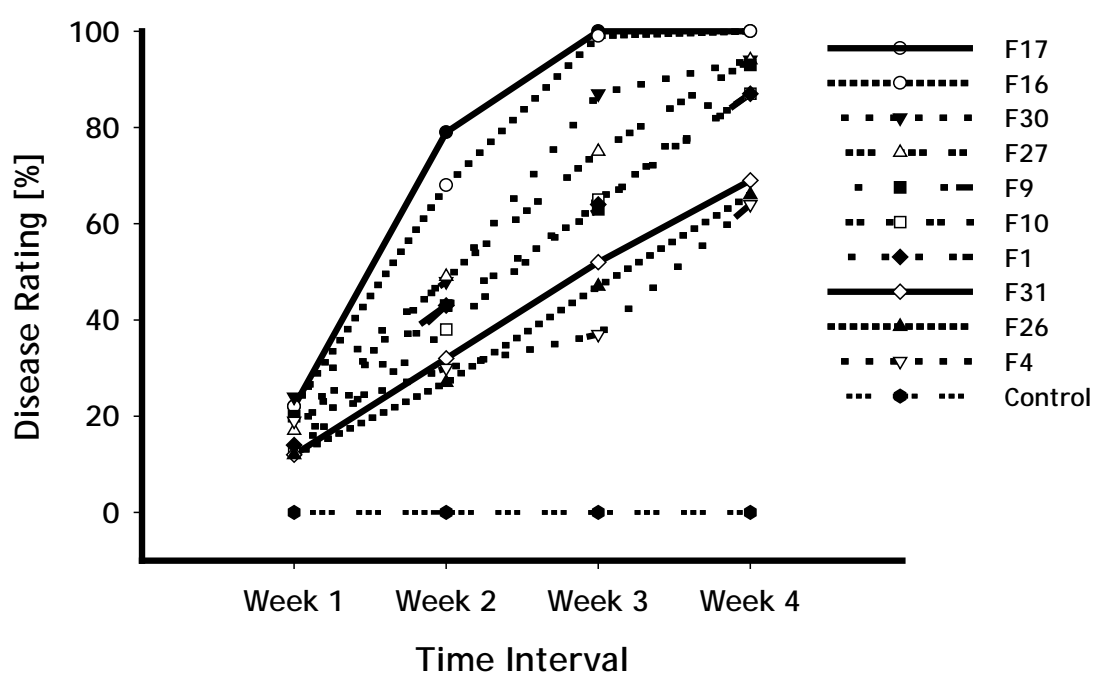

(B)

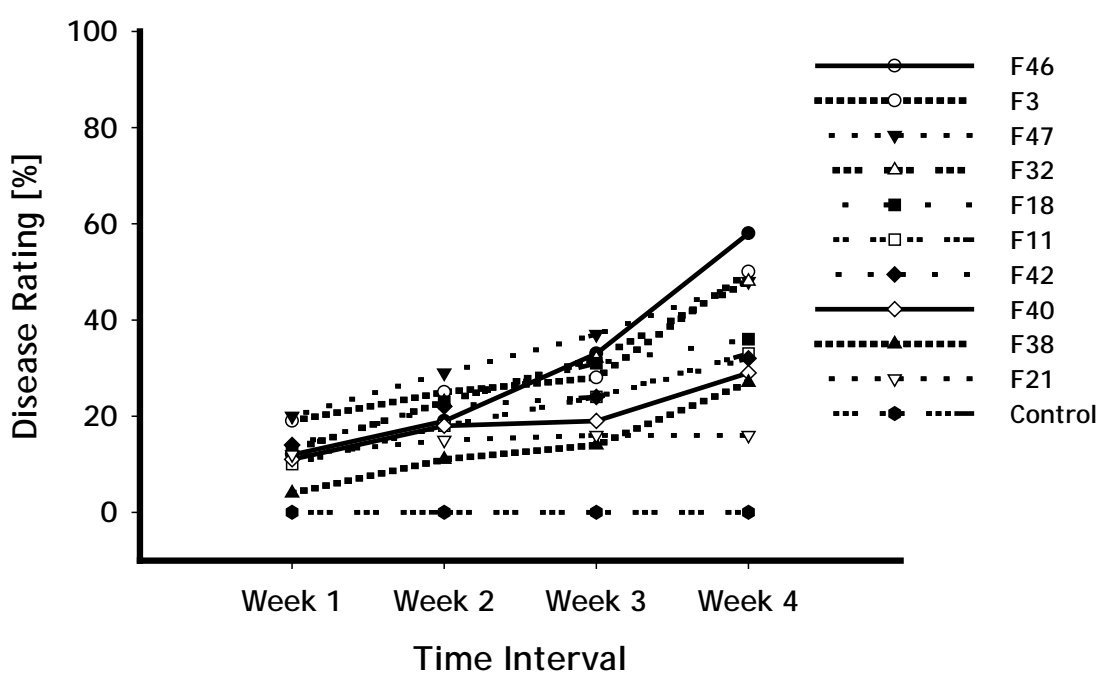

Figure 5. The disease progression on wheat ear infection with different F. graminearum chemotypes over week intervals. The disease progression was rated on 1-100\%(see section infection method). (A) Highly aggressive strains caused FHB; (B) Moderately aggressive strains caused FHB on wheat heads. Each treatment is a pooled mean of 20 individual spikes rated per treatment at harvest. 
The head blight resulted in very heavy accumulation of fungal biomass in infected grains. Number of factors were positively correlated at the significant level at $P \varangle 0.05$. The DNA contents were positively correlated with disease rating at $(r=$ 0.516). However, there was a negative correlation between DNA and kernel weight $(r=-0.474)$ and the number of kernels $(r=-0.501)$ (Table 2). F. graminearum DNA was quantified in all FHB infected samples. The amount of DNA ranged between $1.26-3225 \mu \mathrm{g} / \mathrm{kg}$. There was also some DNA detected in the water inoculated control spikes with the probability of background infection. The heavy infestation of the spikes in some treatments such as F4, F16, F17 and F26 resulted in extremely low amounts of grains at harvest. Most of the seeds were hollowed and with negligent weight but large amounts of fungal DNA was quantified at harvest (Fig.6).

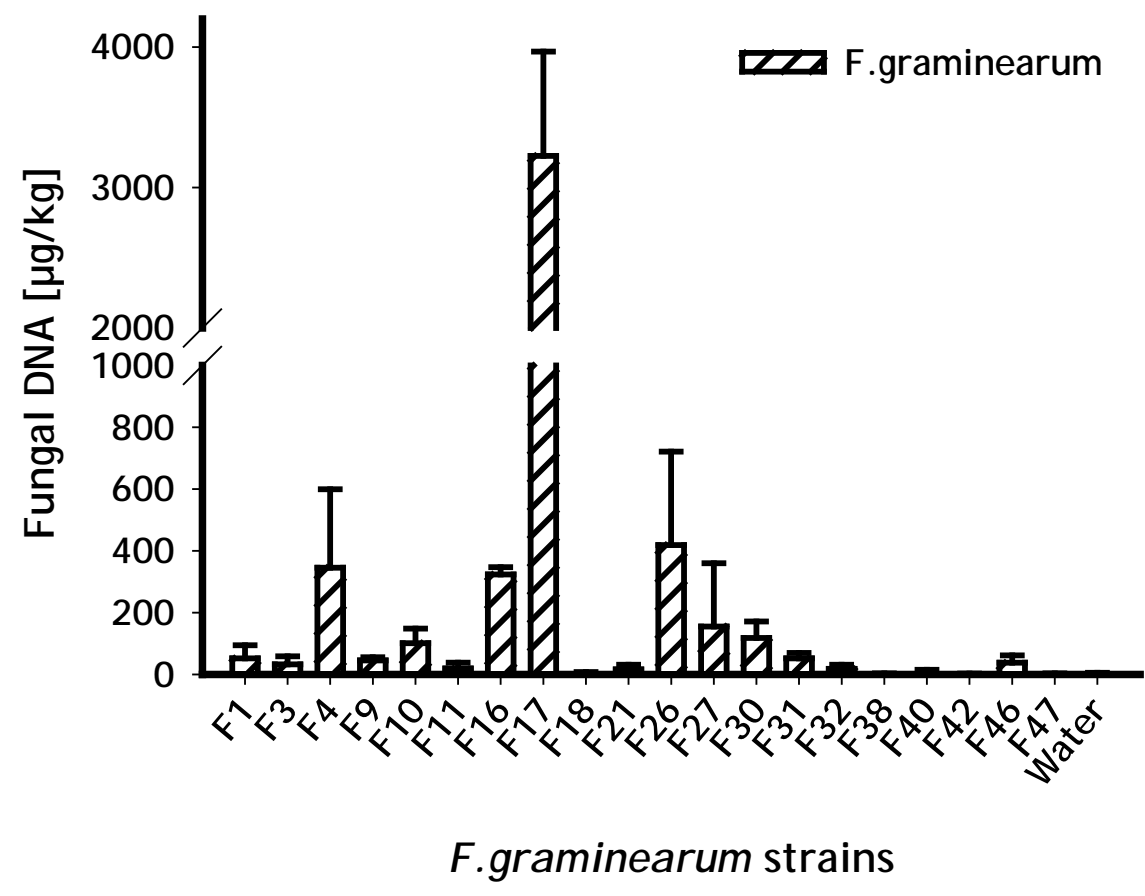

Figure 6. F.graminearum $(\mathrm{Fg})$ DNA quantification with Real Time PCR. The determination of fungal DNA is shown in $\mu \mathrm{g} / \mathrm{kg}$ of total $\mathrm{Fg}$ in FHB infected wheat grains. Numeric with (F) letter denoted to Individual individual F.graminearum strain and control (sterile-waterinoculated heads). Capped bar is a pooled mean of 5 replicates per treatment and a replicate consists of 5 individual spikes with standard deviation 
Table 2. FHB on Apogee wheat heads caused by different F.graminearum strains. Correlations are driven out of Pearson correlations coefficients $(r)$ among DON and other parameters at significant level $(P \varangle 0.05)$ marked with asterisk $(*)$.

\begin{tabular}{llcccccc}
\hline & DON & $\begin{array}{c}\text { Fg } \\
\text { DNA }\end{array}$ & $\begin{array}{c}\text { Disease } \\
\text { rating }\end{array}$ & $\begin{array}{c}\text { No. of } \\
\text { kernels }\end{array}$ & $\begin{array}{c}\text { Kernel } \\
\text { weight }\end{array}$ & NIV & ZEN \\
\hline $\mathbf{1}$ & DON & $0.614^{*}$ & $0.778^{*}$ & $-0.492^{*}$ & $-0.809^{*}$ & & \\
$\mathbf{2}$ & Fg DNA & $0.516^{*}$ & $-0.501^{*}$ & $-0.474^{*}$ & & \\
$\mathbf{3}$ & Disease rating & & $-0.586^{*}$ & $-0.943^{*}$ & & \\
$\mathbf{4}$ & No. of kernels & & & $0.684^{*}$ & & \\
$\mathbf{5}$ & Kernel weight & & & & \\
$\mathbf{6}$ & NIV & & & & \\
$\mathbf{7}$ & ZEN & & & & \\
\hline
\end{tabular}

Most of the inoculated heads with F. graminearum strains turned out to contain large pools of DON, NIV (Fig.7), though the mycotoxins produced varied greatly among strains. DON was the most frequently detected mycotoxin throughout contaminated grains regardless of their chemotype specificity. The quantified DON content among different strains ranged between $0.36-60.81 \mathrm{mg} / \mathrm{kg}$. Therefore, some strains (F4, F18, F21 and F42) could be characterized as NIV producers and quantified as $12.28,6.18,3.14,2.99 \mathrm{mg} / \mathrm{kg}$ respectively. Additionally, few DON and NIV producers also produced ZEN (F3, F21, F40, F42) in lower amounts 0.86, $0.10,0.22,0.02 \mathrm{mg} / \mathrm{kg}$ respectively (Fig.7). Thus, DON producers were more pronounced than NIV producers and the concentration of NIV was lower than DON in contaminated grains. However, NIV accounted the similar level of virulence on spikes as DON producers. Despite differences in the chemotype among these strains, a significant positive correlation between the DON accumulation and fungal DNA contents was tested $(r=0.614, p<0.05)$ (Table 2). The positive correlation was also recorded between DON contents and disease rating at $(r=0.856, P<0.05)$. This correlation was not confirmed in NIV producing strains because of their limited numbers. Additionally, the NIV producers occasionally produced low levels of DON which could also related to the background infection. However, negative correlation was tested among DON, kernel weight and number of kernels at $r=$ 0.809 and $r=-0.492$ respectively $(P \varangle 0.05)$ (Fig. 8). 


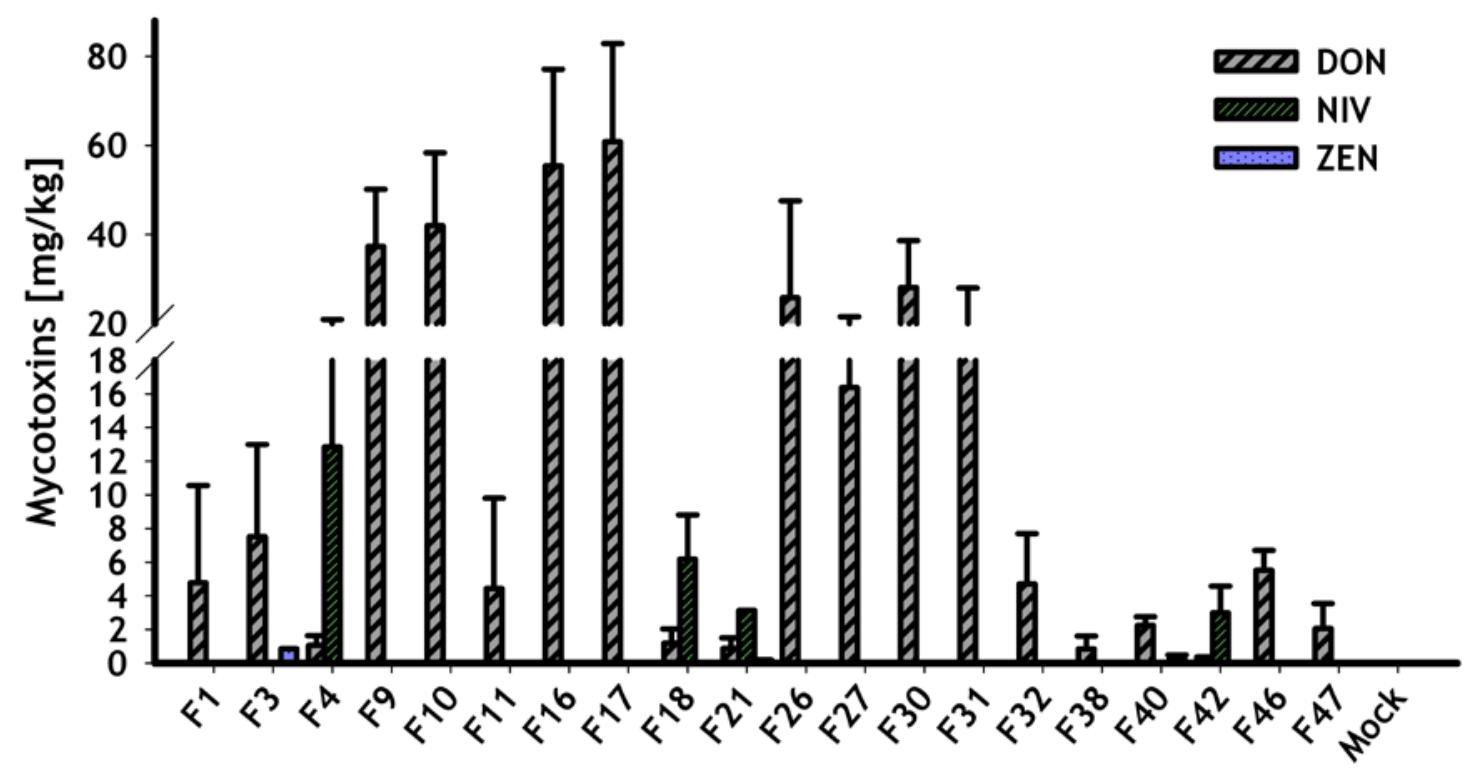

F.graminearum strains

Figure 7. FHB contaminated samples quantified for $F$. graminearum mycotoxin with $\mathrm{HPLC} / \mathrm{MS}-\mathrm{MS}$. Quantification is given in $\mu \mathrm{g} / \mathrm{kg}$ mycotoxins in grounded flour. The numeric with (F) letter denoted for individual F.graminearum strains and mock (water-treated). Capped bar is a pooled mean of 10 replicates per treatment with standard deviation. 
(A)

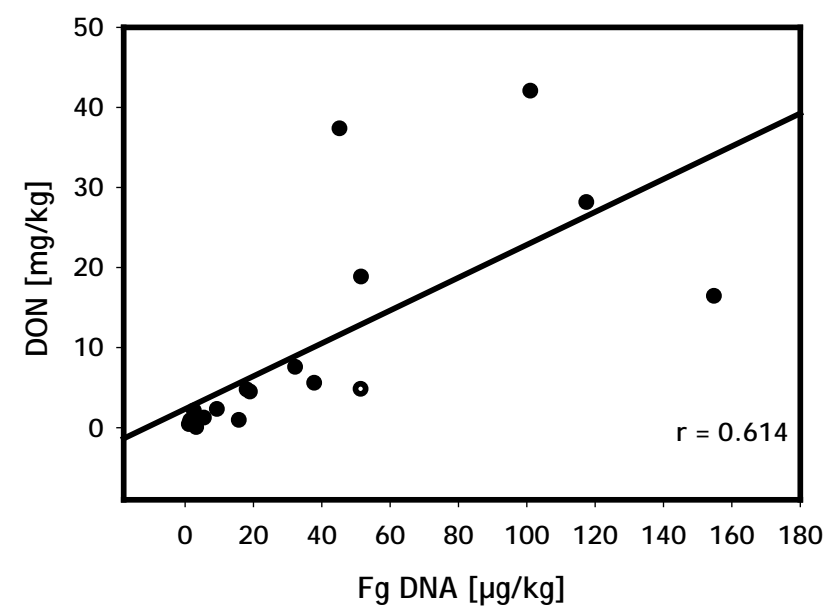

(B)

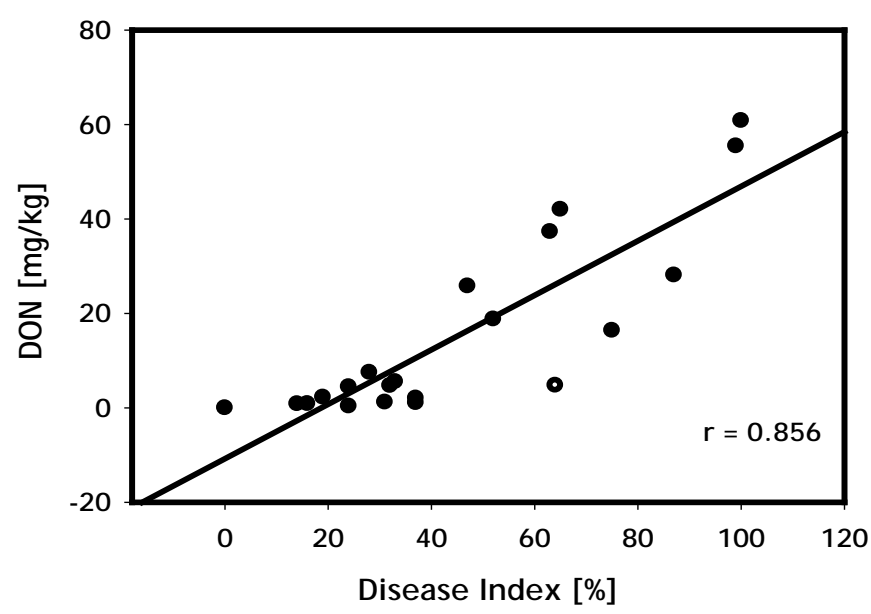

(C)

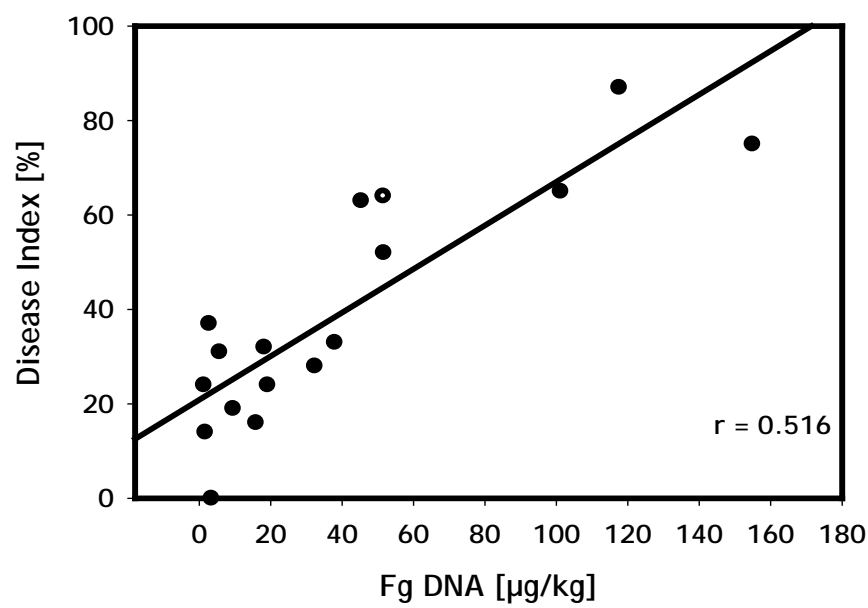

(D)

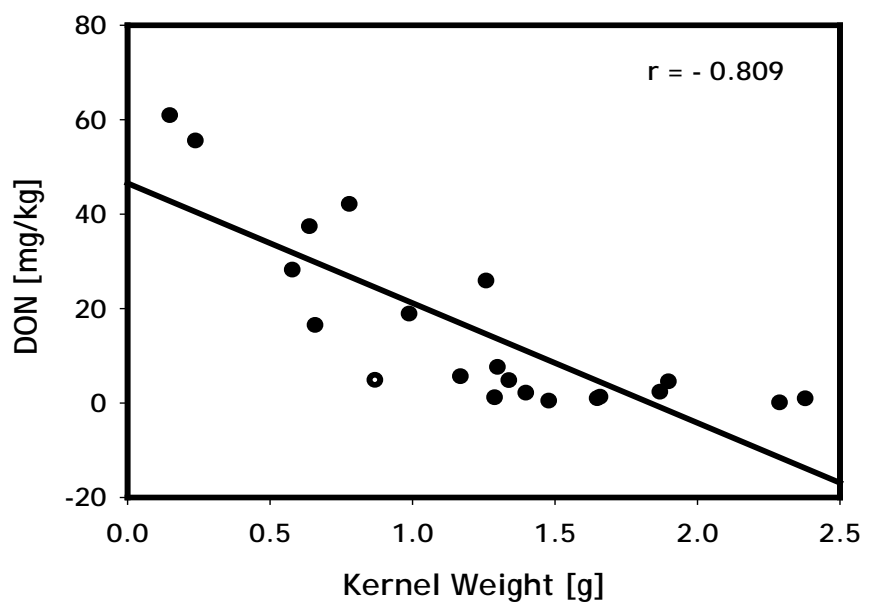

(E)

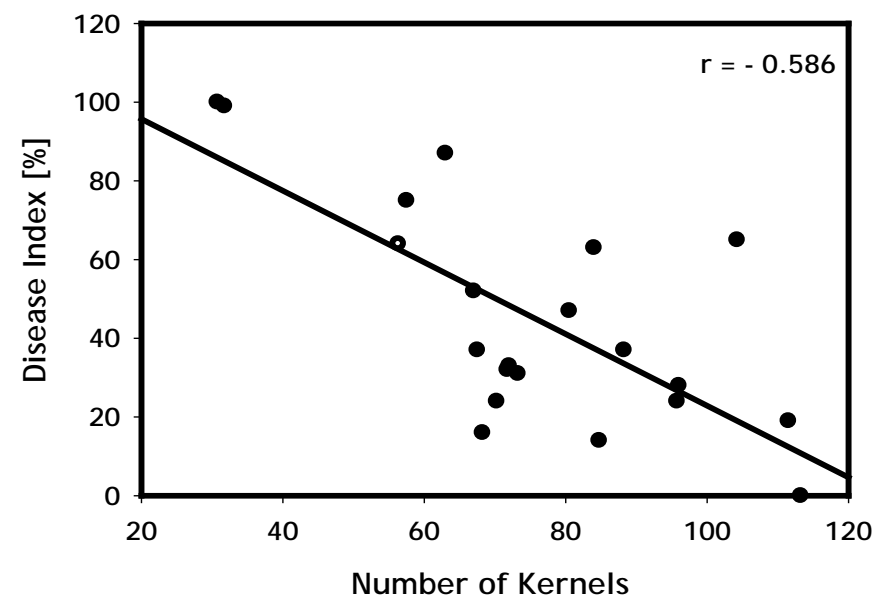

(F)

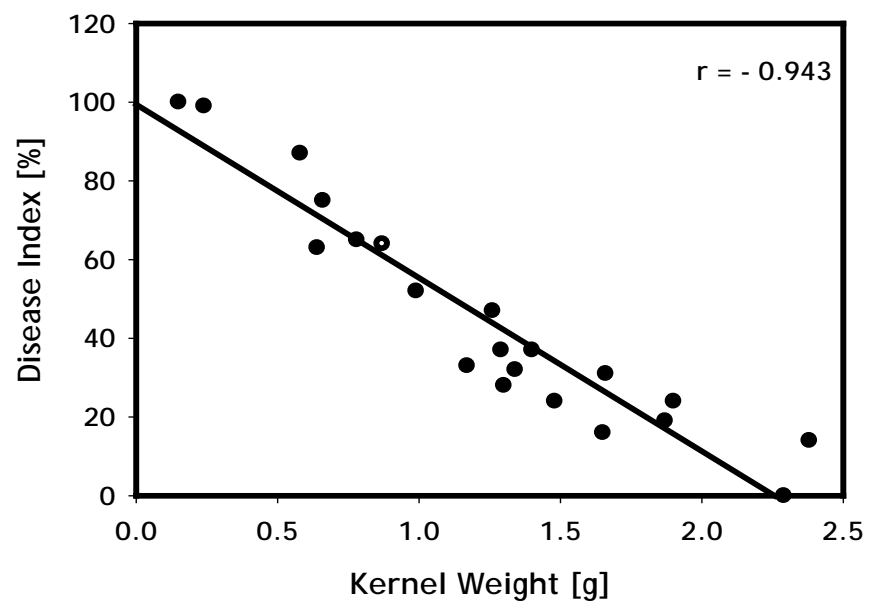

Figure 8. Correlations of FHB symptoms on wheat with different evaluated parameters. A$C$ show positively correlated correlations, D-F show negatively correlated correlations. Correlations were tested with Pearson correlation coefficient at significance level $(\mathrm{P} \bigotimes 0.05)$. 


\section{F. graminearum aggressiveness on maize ears}

Cobs were manually dehusked and rated for the area colonized by the pathogen six weeks post inoculation. We rated each cob for disease severity using the scale 1$100 \%$ (Reid et al. 1996). The huge variation observed among tested strains, showed severe virulence (covered cobs with pink-red mold) to almost no-symptoms on the cob surface area. The disease rating scored between 0 - $63 \%$ among all strains. No visible symptoms were visible on water inoculated cobs or non-inoculated cobs.

A large amount of $F$. graminearum-DNA was quantified in infected grains after disease rating. There was a large variability also recorded among isolates in their mode of action. The DNA contents ranged between $0.3-10559 \mu \mathrm{g} / \mathrm{kg}$ in all strains. The largest amount of DNA was quantified in F4 infected kernels as $10559 \mu \mathrm{g} / \mathrm{kg}$, (which is characterized as NIV producer) (Fig.9).

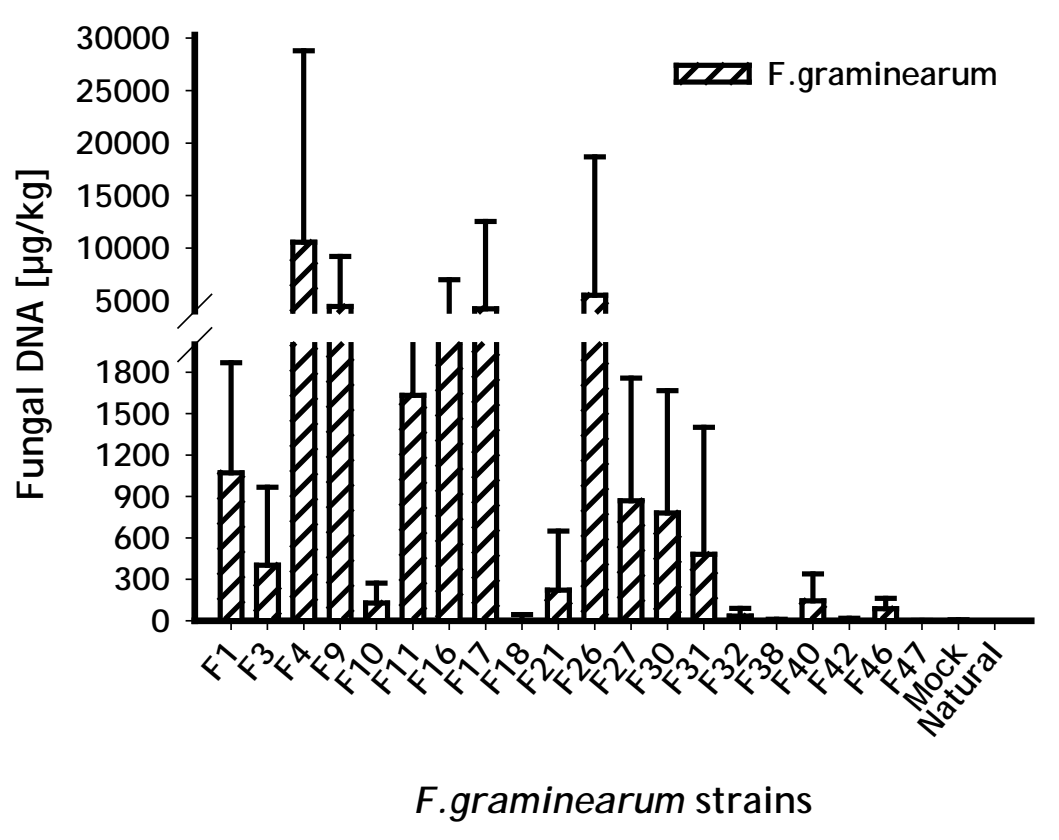

Figure 9. F. graminearum (Fg) DNA quantification with Real Time PCR. Fungal DNA is determined as $\mu \mathrm{g} / \mathrm{kg}$ of total Fg DNA in ear rot infected maize kernels. Numeric with $(F)$ letter denoted to Individual F.graminearum strain number, mock (sterile-water-inoculated ears) and natural (no-fungus). Capped bar is a pooled mean of 10 ears per treatment with standard deviation. 
Table 3. Red ear rot on maize inoculated with different $F$. graminearum strains. Correlations are tested with Pearson correlations coefficients $(r)$ among DON and other parameters. Significant correlations are marked with asterisk $\left(^{*}\right)$ at $(P \varangle 0.05)$.

\begin{tabular}{|c|c|c|c|c|c|c|c|}
\hline DON & 3ADON & NIV & ZEN & $\begin{array}{c}\text { Fg } \\
\text { DNA }\end{array}$ & $\begin{array}{c}\text { Disease } \\
\text { rating }\end{array}$ & $\begin{array}{l}\text { Cob dry } \\
\text { weight }\end{array}$ & $\begin{array}{c}\text { Cob fresh } \\
\text { weight }\end{array}$ \\
\hline $\begin{array}{l}\text { DON } \\
\text { 3ADON } \\
\text { ZEN } \\
\text { Fg DNA } \\
\text { Disease } \\
\text { Cob dry } \\
\text { Cob fres }\end{array}$ & $\begin{array}{l}0.778^{*} \\
\\
\text { rating } \\
\text { weight } \\
\text { h weight }\end{array}$ & & $-0.0194^{*}$ & $-0.107 *$ & $\begin{array}{l}0.834^{*} \\
0.496^{*} \\
-0.0728^{*} \\
0.506^{*}\end{array}$ & $\begin{array}{l}-0.756^{*} \\
-0.376^{*} \\
-0.0973^{*} \\
-0.231^{*} \\
-0.853^{*}\end{array}$ & $\begin{array}{l}-0.694^{*} \\
-0.355^{*} \\
-0.0171^{*} \\
-0.219^{*} \\
-0.820^{*}\end{array}$ \\
\hline
\end{tabular}

All samples were tested positive for the DON content in kernels except F4, F18, F38, and F42 which were previously characterized as NIV chemotype. However, DON was detected in only one replicate of F4 infected sample which could be the background infection. ZEN was quantified throughout all infected samples regardless of their chemotype specificity. The DON quantification ranged between $2.2-90.74 \mathrm{mg} / \mathrm{kg}$. ZEN was quantified between $0.02-5.98 \mathrm{mg} / \mathrm{kg}$. The 3ADON was also frequently detected in infected kernels in many strains except F4, F18, F38, and F42 which were NIV producers. The amount of 3ADON ranged between 1.25 $17.86 \mathrm{mg} / \mathrm{kg}$. In opposite to 3ADON, 15ADON was not so frequently detected. Only the strains F16 and F26 contained 15ADON in detectable quantities of $8.17-11.36$ $\mathrm{mg} / \mathrm{kg}$. The strains F4, F18 and F42 infected kernels contained large amounts of NIV and were quantified as $62.90,15.63$ and $29.60 \mathrm{mg} / \mathrm{kg}$ respectively (Fig. 10). There was a positive correlation tested between DNA contents and disease rating at $(r=0.506, P<0.05)$. Moreover, DON was positively correlated in all strains with disease rating at $(r=0.834, P \varangle 05)$. However, DON was negatively correlated with fresh and dry cob biomass at correlation coefficient $r=-0.694$ and $r=-0.756$, respectively at $(P \ll 0.05)$ (Table 3 ). 
(A)

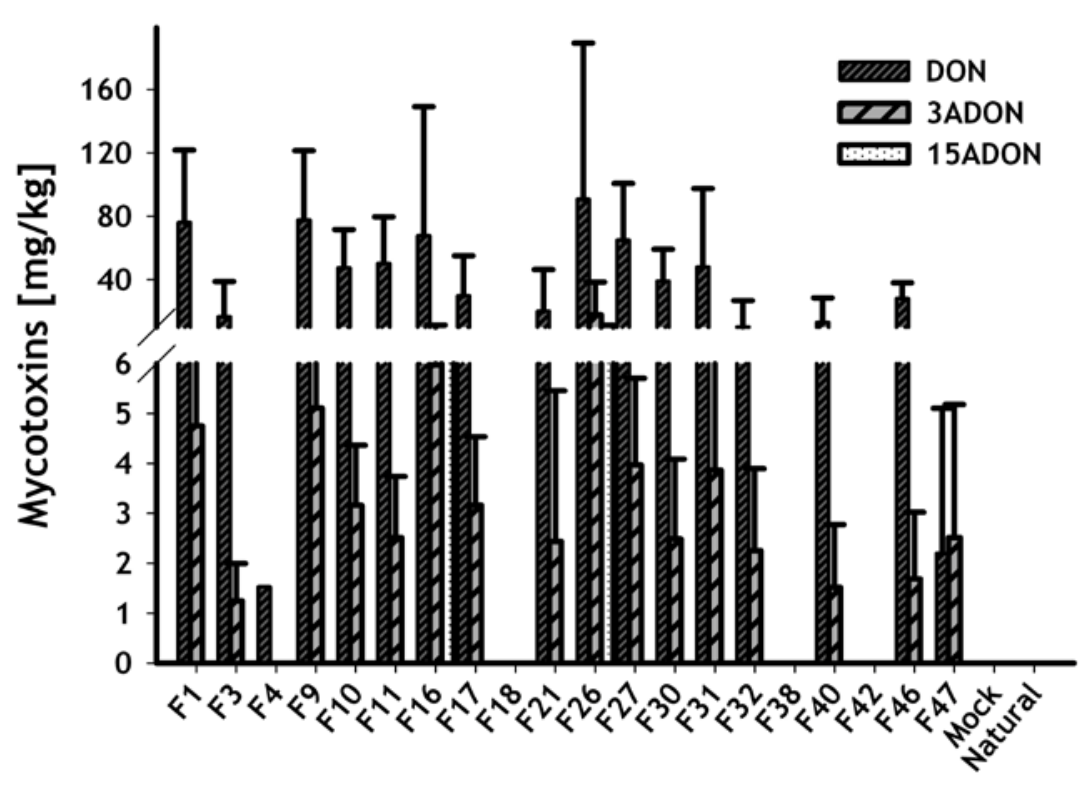

F.graminearum strains

(B)

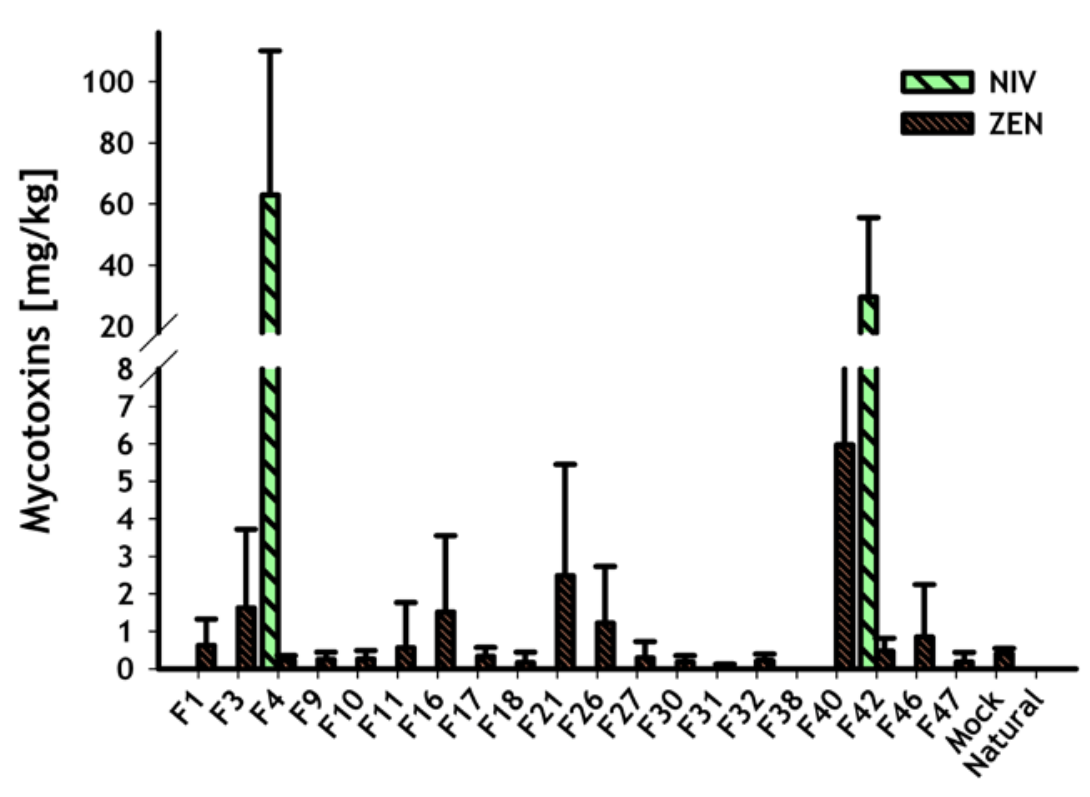

F.graminearum strains

Figure 10. Ear rot of maize infected kernels quantified for mycotoxins analysis with HPLC/ MS-MS. (A) Graph shows DON, 3ADON and 15ADON contents. (B) Graph shows NIV and ZEN contents. Quantification is given in $\mu \mathrm{g} / \mathrm{kg}$ mycotoxins in grounded flour. Numeric with (F) letter denoted to Individual F.graminearum strain number, mock (water-treated) and natural (no-fungus). Capped bar is a pooled mean of 10 ears per treatment with standard deviation. 
(A)

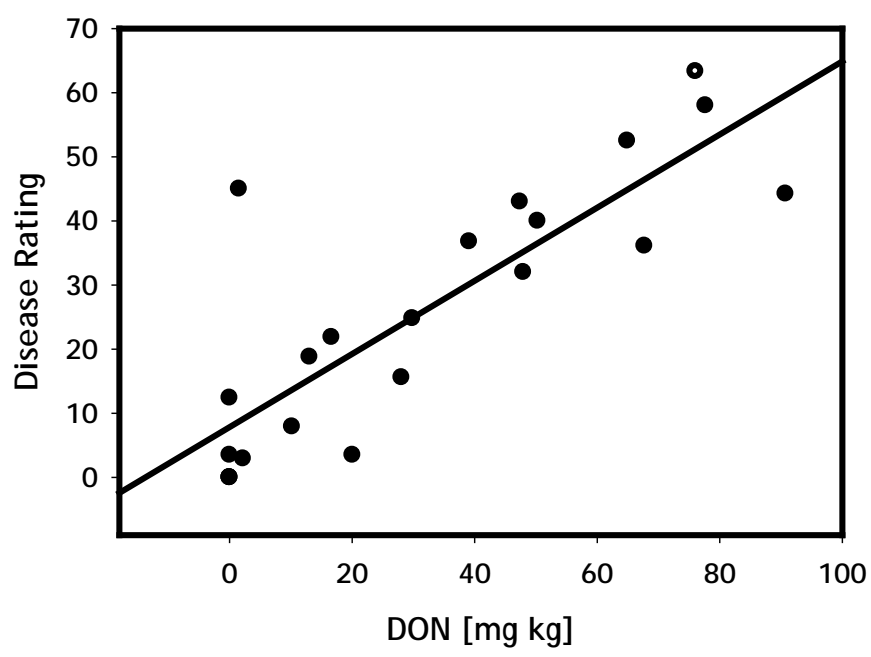

(B)

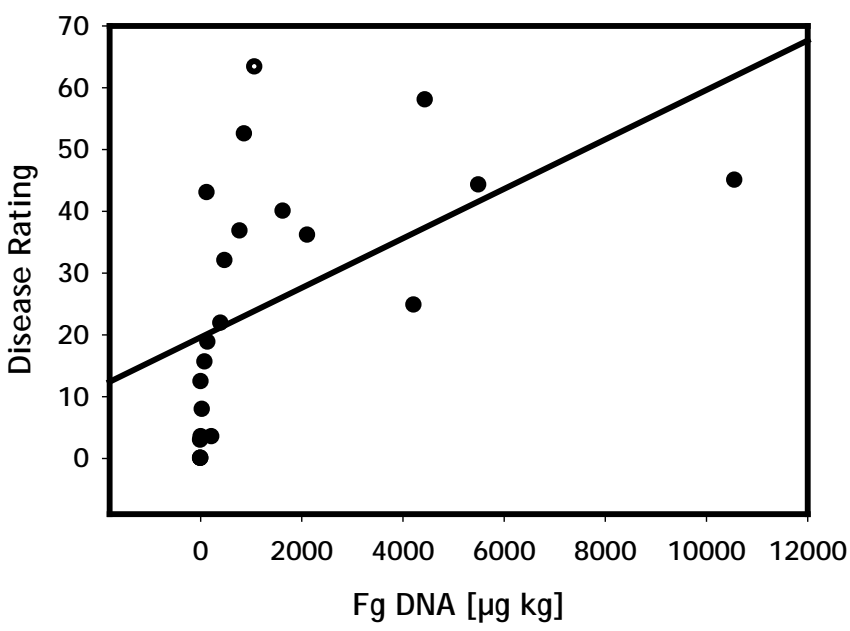

Figure 11. Fusarium ear rot of maize. (A) DON $\mu \mathrm{g} / \mathrm{kg}$ correlated with disease rating, (B) F. graminearum DNA $\mu \mathrm{g} / \mathrm{kg}$ correlated with disease rating tested with Pearson correlation coefficient at significance level $(P \varangle 0.05)$. 


\section{Discussion}

\section{F. graminearum aggressiveness on maize and wheat root}

We investigated the Fusarium-root interactions on maize and wheat to consider the importance of different chemotypes in root infection. Very few studies so far have been carried out to examine the pathogenicity and role of trichothecenes on seedling infection. Wang et al. (2006) reported that F. graminearum DON (Tri5-) deficient mutants produced less virulence compared to wild-type (Tri5t) counterparts on wheat, barley and triticale seedlings infection. (Ludewig et al., 2005) tested the aggressiveness of several F. graminearum isolates on wheat seedlings and found no correlation between toxin production and aggressiveness among isolates. However, the isolates were grown on rice culture to evaluate the DON and other trichothecenes accumulation. In both reported works, either a limited range of isolates were used to evaluate the aggressiveness, or a seedling injury method was used to test the pathogenicity. In our work, a direct seed inoculation method was used in order to reduce the potential injury risk to the seedlings, as this is widely applied in several other host-pathogen studies (Chongo et al., 2001; Jensen et al., 2000; Knudsen et al., 1995; Wang et al., 2006). In our results, the aggressiveness of all tested strains was positively correlated between maize and wheat root infection. Additionally, the strains were found to be more virulent and pathogenic on maize than on wheat. In maize, many seeds were killed at the germination stage after receiving the pathogen inoculum. In this case, the pathogen was re-isolated from several dead seeds to confirm the infection. Maize as a lucrative host for the Fusarium species and has been extensively documented in different regions of the world (Logrieco et al., 2002; Munkvold, 2003; Sutton, 1982). In the experimental work carried out here, the highest level of disease scored on the maize seedlings between $0.2-3.3$ (scale $0=$ healthy and $4=$ dead), regardless of the strains chemotype. Whereas, slightly lower level of disease was scored on wheat seedlings ranged 0.35-2.3. Our results are supported by Carter et al. (2002) who positively correlated the pathogenicity of F. graminearum strains between maize and wheat. Moreover, the disease severity scored higher on maize than wheat seedlings. The involvement of other metabolites in pathogenesis which predominantly govern the initial infection could be one important reason causing 
the seeds of maize and wheat to die before germination. Similar results were obtained by Chongo et al. (2001) who scored the disease severity on wheat seedlings solely based on dead seeds. Number of other studies confirmed the specific role of other metabolites in infection; Adams and Hart (1989) reported several non-toxigenic fusion products highly pathogenic on maize ears and carnation flowers. Pineiro et al. (1995) reported the involvement of other toxins in barley infection. This also indicates that trichothecenes (DON or NIV) are important factors in aggressiveness to maize and wheat (Harris et al., 1999; Maier et al., 2006) but not involved in disease initiation on wheat heads (Bai et al., 2002). The heavy loss of seedling biomass in both shoot and root indexes at harvest could be related to the late production of trichothecenes, which aid the pathogen to accelerate the disease severity as well as the inhibition of protein synthesis in plant cell (Desjardins and Hohn, 1997; Rocha et al., 2005). Consequently, the seedlings which escaped or survived the initial infection showcased partially blighted stand and deformation at harvest. Most of the strains in our study were characterized as DON producers, which endorse the findings of Carter et al. (2002), who observed reduced biomass in seedlings infected with DON producers at harvest and elevated levels of disease.

\section{F. graminearum aggressiveness on wheat heads}

In our Fusarium-wheat infection system, majority of strains were able to produce pathogenicity and were capable of spreading up and downwards from the point of inoculation on the individual spike regardless of their chemotype specificity. Out of twenty tested strains, sixteen were characterized as DON and four as NIV producers. Some strains were able to produce small amount of ZEN in addition to DON. The virulence intensity did not seem fully dependent on the type of trichothecenes produced by individual strains but rather to the amount of toxin accumulated in infected grains. The virulence intensified one week after inoculation and progressed several fold over the third weeks (day 21) up to harvest (Fig. 5). The majority of spikes appeared severely bleached and partially blighted. However, the presence of the pathogen on colonized spikes visually accounted for higher disease rating on the scale. The fungal colonization through disease rating was positively correlated with fungal DNA in infected samples. Similar results were 
obtained by (Miedaner et al., 2000) who tested a tight correlation between ergosterol contents and head blight rating among $\mathrm{F}$. graminearum strains on winter rye. Zhang et al. (2009) also found a strong positive correlation between infected spikelets and DNA contents on winter wheat. Conversely, the correlation was negative for DNA content, kernel weight and number of kernels (Table 2). The elevated levels of DON produced by individual strain remained a decisive factor for pathogen virulence on wheat. Our findings are contrary to Carter et al. (2002) who reported the NIV producers to be more virulent than the DON producers in maize and wheat seedling infection. One important reason of this contradiction could be the investigated systems and cell types and developmental stages of the host plants. Therefore, Carter and co-workers focused on seedlings pathogenicity, whereas present study focused on head blight. Our results are in accordance with previous studies showing that trichothecenes, which have been previously identified as virulence factors (Bai et al., 2002; Maier et al., 2006), have a significant role in determining the aggressiveness of the pathogen. Highly aggressive strains have been found to produce higher levels of trichothecenes irrespective of their chemotype. The fact that NIV has been shown to be more toxic than DON (Ryu et al., 1988), could explain the observation that the maximum amount of trichothecenes quantified from infected spikelets was higher for DON $\left(60.81 \mu \mathrm{g} \mathrm{kg}^{-1}\right)$ than for NIV $\left(12.85 \mu \mathrm{g} \mathrm{kg}^{-1}\right)$ producers with similar levels of aggressiveness. Our results are in line with Goswami and Kistler (2005) who reported higher amounts of DON accumulated in infected wheat heads than NIV among several Fusarium species. The heavy accumulation of DON was tightly correlated with DNA contents in infected grains $(r=0.614)$ and has been confirmed by several other studies (Demeke et al., 2010; Zhang et al., 2009). The amount of trichothecenes measured at day 21 post inoculation was high among several $F$. graminearum strains. Our results are supported by Yoshida and Nakajima (2010) who reported trichothecenes accumulation until 20 days after anthesis in wheat ears.

\section{F. graminearum aggressiveness on maize ears}

In our Fusarium-maize pathosystem, the silk channel inoculation method showed great homogeneity in infection as described earlier in number of studies (Miedaner 
et al., 2010; Reid et al., 1996; Reid et al., 1999). However the purpose of our study was to compare different $F$. graminearum strains for their pathogenicity and mycotoxins accumulation in different host systems rather than screening maize hybrids for resistance. The virulence among strains remained largely variable, scored $(0 \%)$ no colonized symptoms on the kernel surface to $(63 \%)$ partially covered with pink- to reddish-colored mold on kernels that usually spread from the tip of the ear downward or outward to husk. The controls (water-inoculated or noninoculated cobs) showed no pathogen presence.

The large surface area of kernels covered with pathogen accounted for heavy DNA accumulation in infected kernels at harvest. Therefore, the quantified DNA range was between $0.3-10559 \mu \mathrm{g} / \mathrm{kg}$ in all treated strains. The greatest amount of DNA quantified in F4 infected samples (which is a NIV producer). The disease rating was positively correlated with DNA contents in infected kernels (Table 3). The presence of fungal biomass in infected kernels might be a straight precursor of pathogen establishment of post harvest contamination with mycotoxins. Reid et al. (1999) found the highest correlation coefficient between kernel ergosterol contents and disease rating among F. graminearum strains; however a weak correlation was tested for $\mathrm{F}$. moniliforme.

The trichothecene DON was frequently detected, followed by its derivatives 3ADON and 15ADON. However ZEN was the second prevalent mycotoxin detected in all contaminated samples regardless of their chemotype. NIV was quantified among three strains (F4, F18 and F42 characterized as NIV producers) detected in lower amounts than DON. The 3ADON was commonly found in DON producers but not in NIV producers. However, 15ADON was quantified in only two strains F16 and F26 which are potential DON producers. The amount of DON in most of the infected kernels indicates that DON production played a vital role in disease progression in particular and virulence in general to the pathogen. We found a tight correlation between the amount of DON and fungal virulence. In contrast, DON was negatively correlated with fresh and dry cob biomass (Table 3). Harris et al. (1999) reported that in field trials of maize, F. graminearum DON (Tri5-) disrupted mutants were found not to be as virulent as their wild-type (Tri5+) progenitor and DON appeared to act as additional virulence factors in Gibberella ear rot. Numerous field studies on maize have been reported that DON is a core determinant in virulence of $F$. graminearum and a decisive factor for the host specificity (Miedaner et al., 2010; Reid et al., 1996; Schaafsma et al., 1993). However, the role of NIV in fungal 
virulence also can not be ignored. NIV was quantified slightly lower in infected kernels than DON but remained equally aggressive on the cobs. One reason for the lower quantity could be that NIV is 10 -fold more toxic than DON and therefore a relatively small amount could be enough for phytotoxicity (Ryu et al., 1988; Snijders, 1990). Our results support Maier et al. (2006) who reported that F. graminearum (Tri5-) disrupted mutants of NIV-producers on maize cobs were slightly less virulent than wild-type (Tri5t) progenitors. They concluded that NIV appeared to be a virulence factor in the infection of maize cobs. In another study Desjardins et al. (2008) investigated the role of NIV in naturally infected maize kernels with F. graminearum in Nepal and confirmed this conclusion. On contrary, Miedaner et al. (2010) reported that NIV producers are less virulent than DON producers in maize field trials in Germany. The presence of ZEN was quantified in all infected samples regardless of their chemotypes. One reason for the small amount of ZEN in infected kernels could be because it is produced so late at harvest time six-week post inoculation. However, its late production is well documented (Martins and Martins, 2002; Sutton, 1982) and contamination is generally considered to be post harvest or a grain storage problem. The production of 15ADON together with DON and ZEN in two strains could be a cause of great concern. The first report was from (Abbas et al., 1988) in Minnesota, USA, where they found $F$. graminearum strains producing 15ADON together with DON and ZEN. In conclusion we tested a positive correlation between strains virulence on wheat heads and maize cobs and wheat and maize seedling infection. In our Fusariumroot-ear-infection system, the concrete virulence was observed more on maize than wheat. One great reason to explain the phenomena could be the pathogen choice to grow and breed on maize. Whilst vegetative duration among different crop rotation periods, adaptability as niche, host specificity and population distribution could also be important factors for the pathogen variability. It has been documented in several studies that F. graminearum is a large scale produces of mycotoxins including trichothecenes and polyketides. Our results are further cemented through the large genetic variation which exists within strains and population. Our results could frame further windows to design different models for trichothecenes distributions, epidemiology and host specificity. 


\section{References}

1. Abbas, H. K., C. J. Mirocha, R. A. Meronuck, J. D. Pokorny, S. L. Gould, and T. Kommedahl. 1988. Mycotoxins and Fusarium spp associated with infected ears of corn in Minnesota. Applied and Environmental Microbiology 54:19301933.

2. Adams, G. C. and L. P. Hart. 1989. The role of deoxynivalenol and 15acetyldeoxynivalenol in pathogenesis by Gibberella zeae, as elucidated through protoplast fusions between toxigenic and nontoxigenic strains. Phytopathology 79:404-408.

3. Bai, G. H., A. E. Desjardins, and R. D. Plattner. 2002. Deoxynivalenolnonproducing Fusarium graminearum causes initial infection, but does not cause disease spread in wheat spikes. Mycopathologia 153:91-98.

4. Bai, G. H. and G. Shaner. 1996. Variation in Fusarium graminearum and cultivar resistance to wheat scab. Plant Dis. 80:975-979.

5. Brandfass, C. and P. Karlovsky. 2008. Upscaled CTAB-based DNA extraction and real-time PCR assays for Fusarium culmorum and F. graminearum DNA in plant material with reduced sampling error. International Journal of Molecular Sciences 9:2306-2321.

6. Bugbee, B., G. Koerner, R. Albrechtsen, W. Dewey, and S. Clawson. 1997. Registration of 'USU-Apogee' wheat. Crop Science 37:626.

7. Carter, J. P., H. N. Rezanoor, D. Holden, A. E. Desjardins, R. D. Plattner, and P. Nicholson. 2002. Variation in pathogenicity associated with the genetic diversity of Fusarium graminearum. European Journal of Plant Pathology 108:573-583.

8. Chongo, G., B. D. Gossen, H. R. Kutcher, J. Gilbert, T. K. Turkington, M. R. Fernandez, and D. McLaren. 2001. Reaction of seedling roots of 14 crop species to Fusarium graminearum from wheat heads. Canadian J ournal of Plant Pathology-Revue Canadienne de Phytopathologie 23:132-137.

9. Demeke, T., T. Graafenhan, R. M. Clear, A. Phan, I. Ratnayaka, J. Chapados, S. K. Patrick, D. Gaba, C. A. Levesque, and K. A. Seifert. 2010. Development of a specific TaqMan (R) real-time PCR assay for quantification of Fusarium graminearum clade 7 and comparison of fungal biomass determined by PCR vvith deoxynivalenol content in wheat and barley. International J ournal of Food Microbiology 141:45-50.

10. Desjardins, A. E. 2006. Fusarium mycotoxins - Chemistry, genetics, and biology. APS Press, St.Paul, MN.

11. Desjardins, A. E., M. Busman, G. Manandhar, A. M. Jarosz, H. K. Manandhar, and R. H. Proctor. 2008. Gibberella ear rot of maize (Zea mays) in Nepal: Distribution of the mycotoxins nivalenol and deoxynivalenol in naturally and 
experimentally infected maize. Journal of Agricultural and Food Chemistry 56:5428-5436.

12. Desjardins, A. E. and T. M. Hohn. 1997. Mycotoxins in plant pathogenesis. Molecular Plant-Microbe Interactions 10:147-152.

13. Eudes, F., A. Comeau, S. Rioux, and J. Collin. 2000. Phytotoxicity of eight mycotoxins associated with fusarium in wheat head blight. Canadian J ournal of Plant Pathology-Revue Canadienne de Phytopathologie 22:286-292.

14. Gale, L. R., T. J. Ward, V. Balmas, and H. C. Kistler. 2007. Population subdivision of Fusarium graminearum sensu stricto in the upper midwestern United States. Phytopathology 97:1434-1439.

15. Gilbert, J. and W. G. D. Fernando. 2004. Epidemiology and biological control of Gibberella zeae Fusarium graminearum. Canadian Journal of Plant Pathology-Revue Canadienne de Phytopathologie 26:464-472.

16. Goswami, R. S. and H. C. Kistler. 2004. Heading for disaster: Fusarium graminearum on cereal crops. Mol. Plant Pathol. 5:515-525.

17. Goswami, R. S. and H. C. Kistler. 2005. Pathogenicity and in planta mycotoxin accumulation among members of the Fusarium graminearum species complex on wheat and rice. Phytopathology 95:1397-1404.

18. Harris, L. J., A. E. Desjardins, R. D. Plattner, P. Nicholson, G. Butler, J. C. Young, G. Weston, R. H. Proctor, and T. M. Hohn. 1999. Possible role of trichothecene mycotoxins in virulence of Fusarium graminearum on maize. Plant Disease 83: 954-960.

19. J ennings, P., M. E. Coates, K. Walsh, J. A. Turner, and P. Nicholson. 2004. Determination of deoxynivalenol- and nivalenol-producing chemotypes of Fusarium graminearum isolated from wheat crops in England and Wales. Plant Pathology 53:643-652.

20. Jensen, B., I. M. B. Knudsen, and D. F. Jensen. 2000. Biological seed treatment of cereals with fresh and long-term stored formulations of Clonostachys rosea: Biocontrol efficacy against Fusarium culmorum. European J ournal of Plant Pathology 106:233-242.

21. Kimura, M., I. Kaneko, M. Komiyama, A. Takatsuki, H. Koshino, K. Yoneyama, and I. Yamaguchi. 1998. Trichothecene 3-0-acetyltransferase protects both the producing organism and transformed yeast from related mycotoxins - Cloning and characterization of Tri101. Journal of Biological Chemistry 273: 1654-1661.

22. Knudsen, I. M. B., J. Hockenhull, and D. F. J ensen. 1995a. Biocontrol of seedling diseases of barley and wheat caused by Fusarium culmorum and Bipolaris sorokiniana - Effects of selected fungal antagonists on growth and yield components. Plant Pathology 44:467-477.

23. Knudsen, I. M. B., J. Hockenhull, and D. F. J ensen. 1995b. Biocontrol of seedling diseases of barley and wheat caused by Fusarium culmorum and 
Bipolaris sorokiniana - Effects of selected fungal antagonists on growth and yield components. Plant Pathology 44:467-477.

24. Knudsen, I. M. B., J. Hockenhull, and D. F. J ensen. 1995c. Biocontrol of seedling diseases of barley and wheat caused by Fusarium culmorum and Bipolaris sorokiniana - Effects of selected fungal antagonists on growth and yield components. Plant Pathology 44:467-477.

25. Logrieco, A., G. Mule, A. Moretti, and A. Bottalico. 2002. Toxigenic Fusarium species and mycotoxins associated with maize ear rot in Europe. European J ournal of Plant Pathology 108:597-609.

26. Ludewig, A., U. Kabsch, and J. A. Verreet. 2005. Mycotoxin production of 31 Fusarium graminearum isolates and aggressiveness against wheat seedlings. Zeitschrift fur Pflanzenkrankheiten und Pflanzenschutz-Journal of Plant Diseases and Protection 112: 124-133.

27. Maier, F. J., T. Miedaner, B. Hadeler, A. Felk, S. Salomon, M. Lemmens, H. Kassner, and W. Schafer. 2006. Involvement of trichothecenes in fusarioses of wheat, barley and maize evaluated by gene disruption of the trichodiene synthase (Tri5) gene in three field isolates of different chemotype and virulence. Mol. Plant Pathol. 7:449-461.

28. Martins, M. L. and H. M. Martins. 2002. Influence of water activity, temperature and incubation time on the simultaneous production of deoxynivalenol and zearalenone in corn (Zea mays) by Fusarium graminearum. Food Chemistry 79:315-318.

29. Miedaner, T., C. Bolduan, and A. E. Melchinger. 2010. Aggressiveness and mycotoxin production of eight isolates each of Fusarium graminearum and Fusarium verticillioides for ear rot on susceptible and resistant early maize inbred lines. European J ournal of Plant Pathology 127:113-123.

30. Miedaner, T., C. Reinbrecht, and A. G. Schilling. 2000. Association among aggressiveness, fungal colonization, and mycotoxin production of 26 isolates of Fusarium graminearum in winter rye head blight. Zeitschrift fur Pflanzenkrankheiten und Pflanzenschutz-J ournal of Plant Diseases and Protection 107: 124-134.

31. Munkvold, G. P. 2003. Epidemiology of Fusarium diseases and their mycotoxins in maize ears. European J ournal of Plant Pathology 109:705-713.

32. Nganje, W. E., D. A. Bangsund, F. L. Leistritz, W. W. Wilson, and N. M. Tiapo. 2004. Regional economic impacts of Fusarium Head Blight in wheat and barley. Review of Agricultural Economics 26: 332-347.

33. Nicholson, P., D. R. Simpson, G. Weston, H. N. Rezanoor, A. K. Lees, D. W. Parry, and D. Joyce. 1998. Detection and quantification of Fusarium culmorum and Fusarium graminearum in cereals using PCR assays. Physiological and Molecular Plant Pathology 53:17-37.

34. Parry, D. W., P. J enkinson, and L. Mcleod. 1995. Fusarium Ear Blight (Scab) in small grain cereals - A review. Plant Pathology 44:207-238. 
35. Pineiro, M. S., P. M. Scott, and S. R. Kanhere. 1995. Mycotoxin producing potential of Fusarium graminearum isolates from Uruguayan barley. Mycopathologia 132:167-172.

36. Reid, L. M. and R. I. Hamilton. 1996. Effects of inoculation position, timing, macroconidial concentration, and irrigation on resistance of maize to Fusarium graminearum infection through kernels. Canadian J ournal of Plant Pathology-Revue Canadienne de Phytopathologie 18:279-285.

37. Reid, L. M., D. E. Mather, and R. I. Hamilton. 1996. Distribution of deoxynivalenol in Fusarium graminearum infected maize ears. Phytopathology 86:110-114.

38. Reid, L. M., R. W. Nicol, T. Ouellet, M. Savard, J. D. Miller, J. C. Young, D. W. Stewart, and A. W. Schaafsma. 1999. Interaction of Fusarium graminearum and F. moniliforme in maize ears: Disease progress, fungal biomass, and mycotoxin accumulation. Phytopathology 89:1028-1037.

39. Rocha, O., K. Ansari, and F. M. Doohan. 2005. Effects of trichothecene mycotoxins on eukaryotic cells: A review. Food Additives and Contaminants 22:369-378.

40. Ryu, J. C., K. Ohtsubo, N. Izumiyama, K. Nakamura, T. Tanaka, H. Yamamura, and Y. Ueno. 1988. The acute and chronic toxicities of nivalenol in mice. Fundamental and Applied Toxicology 11:38-47.

41. Schaafsma, A. W., J. D. Fuentes, T. J. Gillespie, G. H. Whitfield, and C. R. Ellis. 1993. Performance of a model for egg hatching of the western corn rootworm, Diabrotica virgifera Leconte, using measured and modeled soil temperatures as input. International J ournal of Biometeorology 37:11-18.

42. Schroeder, H. W. and J. J. Christensen. 1963. Factors affecting resistance of wheat to scab caused by Gibberella Zeae. Phytopathology 53:831.

43. Snijders, C. H. A. 1990. Fusarium head blight and mycotoxin contamination of wheat - A review. Netherlands J ournal of Plant Pathology 96: 187-198.

44. Stack, R. and M. McMullan. 1995. A visual scale to estimate severity of Fusarium head blight of wheat. (http:// www. ext. nodak. edu/ extpub/ plantsci/ smgrains/ pp1095w. html).

45. Sutton, J. C. 1982. Epidemiology of wheat head blight and maize ear rot caused by Fusarium graminearum. Canadian Journal of Plant Pathology 4: 195.

46. Vaneeuwijk, F. A., A. Mesterhazy, C. I. Kling, P. Ruckenbauer, L. Saur, H. Burstmayr, M. Lemmens, L. C. P. Keizer, N. Maurin, and C. H. A. Snij ders. 1995. Assessing nonspecificity of resistance in wheat to head blight caused by inoculation with european strains of Fusarium culmorum, F. graminearum and $F$. nivale using a multiplicative model for interaction. Theoretical and Applied Genetics 90:221-228. 
47. Wang, H., S. F. Hwang, F. Eudes, K. F. Chang, R. J. Howard, and G. D. Turnbull. 2006. Trichothecenes and aggressiveness of Fusarium graminearum causing seedling blight and root rot in cereals. Plant Pathology 55:224-230.

48. Ward, T. J., J. P. Bielawski, H. C. Kistler, E. Sullivan, and K. O'Donnell. 2002. Ancestral polymorphism and adaptive evolution in the trichothecene mycotoxin gene cluster of phytopathogenic Fusarium. Proceedings of the National Academy of Sciences of the United States of America 99:9278-9283.

49. Xu, X. M., D. W. Parry, P. Nicholson, M. A. Thomsett, D. Simpson, S. G. Edwards, B. M. Cooke, F. M. Doohan, J. M. Brennan, A. Moretti, G. Tocco, G. Mule, L. Hornok, G. Giczey, and J. Tatnell. 2005. Predominance and association of pathogenic fungi causing Fusarium ear blight in wheat in four European countries. European J ournal of Plant Pathology 112: 143-154.

50. Yoshida, M. and T. Nakajima. 2010. Deoxynivalenol and nivalenol accumulation in wheat infected with Fusarium graminearum during grain development. Phytopathology 100:763-773.

51. Zhang, Y. J., P. S. Fan, X. Zhang, C. J. Chen, and M. G. Zhou. 2009. Quantification of Fusarium graminearum in harvested grain by real-time polymerase chain reaction to assess efficacies of fungicides on Fusarium head blight, deoxynivalenol contamination, and yield of winter wheat. Phytopathology 99:95-100. 


\title{
Chapter 4: Role of deoxynivalenol (DON) and nivalenol (NIV) in wheat and maize root infection by F. graminearum
}

\author{
Awais Ahmed, Stephan Bucher, Katharina Döll and Petr Karlovsky \\ Molecular Phytopathology \& Mycotoxin Research Unit, Georg-August University, \\ Goettingen, Germany.
}

\section{Abstract}

F. graminearum is an important toxigenic pathogen which causes head blight and root rot in wheat and ear rot and seedling blight in maize. The trichothecenes type B deoxynivalenol (DON) and nivalenol (NIV) are major mycotoxins produced by the fungus. We investigated the role of DON and NIV in wheat and maize root infection. We inoculated the seeds of either wheat or maize with two F. graminearum wildtype strains specific to DON and NIV chemotypes and two individual deficient mutants corresponding to each chemotype. Wheat inoculated with NIV wild-type showed poor emergence, reduced root and shoot indexes and increased disease severity compared to the deficient mutants. The DNA content showed a larger amount of fungal DNA in NIV wild-type infected root or shoot but an only trace of fungal DNA was detected in deficient mutants. NIV was detected in traceable amounts but with great variability among samples of wheat or below the detection limits. Maize inoculated with either NIV wild-type or deficient mutants produced the same amount of disease. DON wild-type inoculated wheat suffered extreme losses in germination, resulted in reduced biomass indexes and greater disease severity than deficient mutants. No significant differences were observed between DON wild-type and deficient mutants inoculated maize. We conclude that NIV and DON are virulent factors on wheat not on maize root infection. 


\section{Introduction}

F. graminearum (Schwabe) is the most frequently associated pathogen with Fusarium head blight (FHB) disease in numerous cereal crops. Being a commonly recognized FHB pathogen, its aggressiveness towards seedling blight and rot in wheat and maize has also been reported (Parry et al., 1995; Sutton, 1982). Due to its complex biology, an interchangeable saprophytic and necrophytic phase during its life cycle, makes it a multi-faceted pathogen. Maize stubbles, decomposed corn cobs and kernels serve as a potential reservoir for this pathogen; where it survives as mycelia and disperse to appropriate host or substrate during the vegetation period (Gilbert and Fernando, 2004; Sutton, 1982). The F. graminearum outbreaks are massive in commercial agriculture production systems, where wheat and maize are usually cultivated in rotations. Up to now FHB epidemics create unprecedented fiscal drains in the United States, China and some parts of Europe, along with Fusarium ear rot disease (Nganje et al., 2004). Contamination with mycotoxins produced by Fusaria is a potential threat that downgrades the agriculture products and is toxic to human and animal consumption. Due to its large chemotype diversity, it triggers the aggressiveness many-fold and facilitates the substrate colonization during infection and at storage conditions. Mycotoxins such as trichothecenes type B group, deoxynivalenol (DON) and its acetylated derivatives 3-acetyldeoxynivalenol (3ADON), 15-acetyldeoxynivalenol (15ADON) and nivalenol (NIV) as well as the estrogenic zearalenone (ZEN) are frequently encountered in cereals and maize (Desjardins and Hohn, 1997). DON accumulates more often in wheat with traceable proportions of 3ADON and 15ADON. The 3ADON or 15ADON are relatively less toxic to mammals than DON (Kimura et al., 1998), but may be equally toxic to plants (Eudes et al., 2000). However, NIV can be a potent mycotoxin in maize and is recognized as ten-fold more toxic than DON or its acetylated derivatives (Ryu et al., 1988; Snijders, 1990). The occurrence of DON and NIV is decisive in wheat and maize infection. This indicates that there is some selective advantage or chemotype balance operates during different phases of pathogen life cycle (Ward et al., 2002). The DON and NIV coexist within species and specific to strain chemotype but their proportions highly fluctuate with seasonal rotations (J ennings et al., 2004). Not enough research is devoted to the investigation of F. graminearum maize and wheat seedling infections. In 
epidemiological studies, (Chongo et al., 2001) tested F. graminearum strains, which were isolated from FHB symptomatic plants on different cereal crops to serve as alternative host. Ludewig et al. (2005) investigated the DON production and aggressiveness by inoculating $31 \mathrm{~F}$. graminearum isolates on wheat seedlings and measured DON contents on rice cultures.

In genetic variation of isolates, Carter et al. (2002) evaluated F. graminearum aggressiveness on maize and wheat seedlings. In axenic cultures, coleoptile's elongation and root growth of wheat seedlings were inhibited by DON, whereas NIV was ineffective at similar concentrations (Eudes et al., 2000; Shimada and Otani, 1990).

The specific role of DON and NIV was tested in some gene-knock-out studies. The DON (Tri5-) disrupted mutants of $F$. graminearum were inhibited in DON biosynthesis and showed less aggressiveness on wheat (Proctor et al., 1995). The DON (Tri5-) disrupted mutants of F. graminearum were unable to spread beyond the point of inoculation on wheat unlike its progenitors (Bai et al., 2002). In maize field trials, DON (Tri5-) disrupted mutants were not as virulent as its progenitors in causing Gibberella ear rot (Harris et al., 1999). However, Maier et al. (2006) indicated that F. graminearum NIV (Tri5-) has little or no effect on wheat ear infection, but it appeared to be a virulence factor in maize cobs.

In seedling inoculation, DON (Tri5-) disrupted mutants were less virulent on cV. Wheaton and an unknown winter rye and no effect was observed on Golden Bantam maize (Proctor et al., 1995). The role of DON (Tri5-) disrupted mutants was also evaluated on wheat, barley and triticale seedlings infection, where Wang et al. (2006) found the DON (Tri5-) disrupted mutant less aggressive than progenitors.

DON is a decisive factor for $F$. graminearum aggressiveness during infection but the specific role of NIV in cereals and maize seedling infection has not been investigated yet. We tested the role of F. graminearum NIV (Tri5t) and DON (Tri5+) chemotype together with their two independent NIV (Tri5-) and DON (Tri5-) mutants generated in the cited work (Maier et al. 2006). The objectives of this study were: (1) to test the virulence of the NIV and DON chemotype and the respective deficient mutants on wheat and maize seedlings and (2) to quantify DNA contents and mycotoxin accumulation in infected samples. 


\section{Material and Methods}

Table 1. Assigned code, host species/organ, country and original code of Fusarium graminearum wild-type and knock-out strains used in this study.

\begin{tabular}{llll}
\hline Assigned code $^{\mathbf{a}}$ & Host species/ organ & Country & Original code $^{\mathbf{b}}$ \\
\hline Fg NIV Wt & Rye, kernel & Germany & Fg 06 Wt \\
Fg NIV Deficient 1 & & & $\mathrm{Fg} \mathrm{06 \# 7}$ \\
Fg NIV Deficient 2 & Wheat, head & Germany & $\mathrm{Fg} \mathrm{06 \# 10}$ \\
Fg DON Wt & & $\mathrm{Fg} \mathrm{2311} \mathrm{Wt}$ \\
Fg DON Deficient 1 & & $\mathrm{Fg} \mathrm{2311 \# 2899}$ \\
Fg DON Deficient 2 & & & \\
\hline
\end{tabular}

(a) Both Fg (wt) strains denoted as NIV (Tri5t) and DON (Tri5+) and deficient mutants as NIV (Tri5-) and DON (Tri5-). (b) Fg Wt strains originally isolated by Thomas Miedaner (University of Hohenheim, Germany). The gene-knock-outs strains were produced by Frank Maier (University of Hamburg, Germany).

\section{Fungal strains, culture conditions and media}

The description of aggressiveness of the strains either NIV or DON producers were ranked in previous studies (Miedaner et al., 2000). The gene-knock-outs (Tri5-) disrupted mutants, generated and tested in the work of (Maier et al., 2006), are listed in (Table 1). Strains were maintained as spore suspension in a mixture of 15 $\%$ glycerol and tap water and frozen in $-80^{\circ} \mathrm{C}$ until use. Fungal inoculum was produced on mung bean liquid medium slightly modified from (Bai and Shaner, 1996) by injecting the $50 \mu \mathrm{l}$ of spore suspension into an Erlenmeyer flask with 100 $\mathrm{ml}$ of medium for four days at $28^{\circ} \mathrm{C}$ with shaking conditions at $200 \mathrm{rpm}$. Spores were filtered through a cotton ball placed directly into a funnel at the top of the Erlenmeyer flask. This was transferred to a falcon tube and centrifuged for 10 minutes at $4500 \mathrm{rpm}$. The supernatant was discarded carefully from the falcon tube. The pellet was re-suspended in sterile tap water. The fungal spore's concentration was determined by placing a few $\mu$ of spore suspension in a Thoma counting chamber. The final concentration was adjusted to $1.5^{*} 10^{5} / \mathrm{ml}$ for seed inoculation. 


\section{Seedling growth conditions}

The cV. Apogee Triticum aestivum 'USU-Apogee' (which is highly susceptible to F. graminearum infection) was obtained from the Federal Government Institute Darmstadt, Germany and originally published in (Bugbee et al., 1997). The Mini maize (Gaspe Flint) is an early flowering, short, yellow-seeded landrace of the Northern Flint race, originally collected in Quebec, Canada, and was provided by Prof. Jan Schirawski, Department of molecular biology of plant-microbe interaction, University of Goettingen, Germany. Both cultivars are designated as moderately susceptible to $\mathrm{F}$. graminearum infection. Both cultivars were grown during pathogenicity tests in a greenhouse at approximately $24 \pm 3^{\circ} \mathrm{C}$ with $16 \mathrm{~h}$ supplemental light (400W Philips bulbs with PIA technology) with the relative humidity between $40-60 \%$ Seedlings were watered daily and fertilized on weekintervals with NPK (15-11-15).

\section{Aggressiveness test on wheat and maize}

To achieve the maximum infection on seeds, we used similar infection methods for both wheat and maize seedlings. The pathogen inoculum was produced in mung bean medium as described in the previous section and $0.5 \%$ xanthan (Xanthomonas campestris, Fluka-Buchs, Switzerland) was incorporated into the inoculum for spore's adhesion to seeds before inoculation. The following codes were designated to different treatments in both wheat and maize greenhouse experiments. The wild type strains were denoted as NIV (Tri5t) and DON (Tri5t) and the deficient mutants as NIV (Tri5-) and DON (Tri5-), respectively. The negative control consisted of water inoculated seeds (sterile water incorporated with xanthan). A single seed was planted in a mixture of plant substrate + sand ratio (2:1) in $4.5 \times 5 \mathrm{~cm} \varnothing$ plastic pots for either maize or wheat. The emulsified pathogen inoculum with xanthan and the macroconidia in a concentration of $1.5 * 10^{5}$ spores/ $\mathrm{ml}$ was directly inoculated onto the seed, according to method modified from (Knudsen et al., 1995). The experiment was conducted in a completely randomized block design with 20 replicates used for each treatment and the experiment was repeated twice under the same conditions in different months. The disease index (DI) was scored at harvest, using the disease scale DI described by (Knudsen et al., 1995) with 
slight modification where 0 - no disease symptoms; 1 - brown pin point lesions on the coleoptile and/or roots; 2 - extended browning of the coleoptile and/or browning/reddening of the roots; 3 - dead coleoptile and/or extended browning/ reddening of the roots; and 4 - browning extending beyond the coleoptile on the above-ground plant parts or dead plant or ungerminated kernel. The percentage of emerged plants was calculated after one week and final seedling stand was evaluated at harvest. Several other parameters such as seedling shoot and root fresh and dry biomass and final seedling survival and seedling stand were also recorded at harvest. 20 replicates were used for each treatment.

\section{Sample plant material}

The seedlings roots were washed and fresh weight was measured. The sample preparation was done through plant material (shoot or root) lyophilization. For this, samples were placed in lyophilizer (Martin Christ, Osterode am Harz, Germany) for two days (ca. 48 hours). The lyophilized material was weighed individually for each sample either shoot or root. Because of the high variation in the sample amount, the whole sample was milled in a small non-commercial mill (Type MM 2, Retsch, Germany) for 30 seconds. The ground material was transferred into $2 \mathrm{ml}$ reaction tubes to extract fungal DNA and mycotoxins.

\section{Fungal DNA extraction (CTAB method)}

The pulverized plant sample was extracted with $1 \mathrm{ml}$ of CTAB buffer according to (Brandfass and Karlovsky, 2008). The dried pellet was dissolved in $50 \mu \mathrm{l} \mathrm{TE}$ buffer. The DNA was checked for both quality and quantity through agarose gel electrophoresis before DNA quantification with Real Time PCR.

\section{F. graminearum DNA quantification by Real Time PCR}

The Real-time PCR DNA quantification was done from protocol (Brandfass and Karlovsky, 2008) and based on primers specific for F. graminearum described by (Nicholson et al., 1998). The fluorescence was measured after each PCR step on 
iCycler (BioRad, Hercules, CA, USA) by using Absolute ${ }^{T M}$ Blue QPCR SYBR GREEN Fluorescein Mix (Thermo Start ${ }^{\mathrm{TM}}$ DNA-Polymerase, $3 \mathrm{mM} \mathrm{MgCl}$, dNTP Mixture, SYBR Green I, and $10 \mathrm{nM}$ Fluorescein, Abgene Limited, United Kingdom). Calibration curve was carried out with purified F. graminearum DNA in $0.5 \mathrm{pg}, 5 \mathrm{pg}, 50 \mathrm{pg}$ and $500 \mathrm{pg}$ added to healthy plant shoot or root DNA. PCR product ratios were converted to DNA concentrations (picograms of total DNA), then to microgram per kilogram $(\mu \mathrm{g} / \mathrm{kg})$ plant material.

\section{Mycotoxin extraction}

The mycotoxins extraction was done according to the following procedure: The lyophilized seedling biomass, either roots or shoots, were extracted according to different groups which correspond to the different weights, because of the high variability among the sample weights. All samples were extracted overnight, shaken in a 1:10 ratio with methanol/isopropanol/ water 80:5:15. The samples were centrifuged at $4500 \mathrm{rpm}$ for $10 \mathrm{~min}$, and one millilitre of supernatant was transferred to a $2 \mathrm{ml}$ reaction tube, evaporated to dryness in a vacuum concentrator (Martin Christ, Osterode, Germany), redissolved in $1 \mathrm{ml}$ methanol/water (50/50), and defatted with $500 \mu \mathrm{l}$ of hexane. Then the samples were centrifuged again at maximum rpm and the lower phases were taken up carefully and transferred to new reaction tubes. All samples were diluted 1:10 with methanol/ water (50/50). An amount of $600 \mu \mathrm{l}$ was transferred into vials for HPLCMS MS. The samples were stored at $-20^{\circ} \mathrm{C}$ until mycotoxin analysis was done.

\section{Mycotoxin analysis (HPLC-MS/MS)}

The samples were analyzed for the content of the type B trichothecenes NIV and DON with HPLC-MS/MS. The system used for determination of the toxins consisted of ProStar 210 pumps, column oven Mistral 510 and 1200 LC Triple Quadrupole mass spectrometer with electrospray ionisation-interface (all Varian, Darmstadt, Germany). For separating, a Kinetex C18 column $(50 \times 2,1 \mathrm{~mm}, 2.6 \mu \mathrm{m})$ (Phenomenex, Aschaffenburg, Germany) was used Standards for calibration curves were prepared for each toxin with spiked blank samples, which were extracted in 
the same way than the samples. For analysis the HPLC-MS/MS detection method developed by Limsuwan et al. (in preparation) was used. The final toxin concentrations were determined by comparison the integrated chromatographic intensities of each sample with the standard curves of each toxin.

\section{Statistical Analysis}

The data was analyzed by Kruskal-Wallis One Way Analysis of Variance on Ranks at $(P=\varangle .05)$ and pairwise multiple comparison was done with (Tukey Test). The Pearson Product Moment Correlation was used to test the correlation among NIV (Tri5+)/ (Tri5-), DNA contents and disease index. Statistical analysis was performed by using the software package "Sigma plot 11.0" (Systat Software, Inc., San J ose, USA).

\section{Results}

\section{F. graminearum aggressiveness on wheat (cv. Apogee) root}

After seeds inoculation on CV. Apogee both NIV (Tri5+) and DON (Tri5+) strains produced initial infection at the seed level (pathogenicity), but swift disease progression observed at 7 days post inoculation (dpi) when seedling blights symptoms showed consistent presence. The marked significant difference was scored in seedlings emergence between (Tri5t) and (Tri5-). The stable seedling emergence was observed in NIV (Tri5-) seeds, however emergence was slightly reduced in DON (Tri5-) inoculated seeds. The severe seedling blights reduced or inhibited germination. Severe browning of coleoptile and browning or reddening on upper and lower parts of roots were frequently observed in NIV (Tri5t) and DON (Tri5t) inoculated seeds and few symptoms on those inoculated with mutants, too.

The NIV (Tri5t) infected seedlings showed greater disease symptoms which resulted in elevated levels of severity and scored higher 3.2 on the disease scale ( $0=$ healthy and $4=$ dead seed), however NIV (Tri5-) inoculated seeds appeared as 
mild symptoms and lower disease severity was scored with the indexes 2.12 and 2.10 on the scale (Fig. 1).

The DON (Tri5t) inoculated seedlings scored greater than NIV (Tri5+) on the disease scale; however, disease severity determination was largely based on dead or killed seeds and scored 3.8 on the scale. The disease severity was rated quite moderate on DON (Tri5-) inoculated seeds with a range of 2.7 and 2.8 on the disease scale (Fig. 1). Some random disease symptoms were also observed on water inoculated seedlings (controls) and resulted 0.22 on the scale. However, some seedlings scored 4 on the scale because they failed to germinate or suffered back ground infection. Some spots of discoloration and browning on coleoptile were observed on some control plants. 
(A)

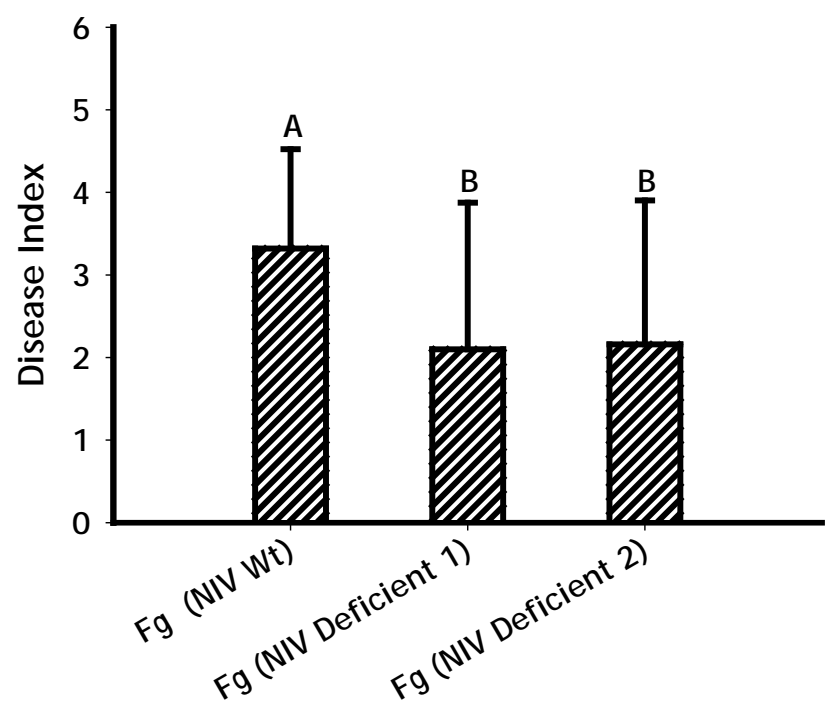

(B)

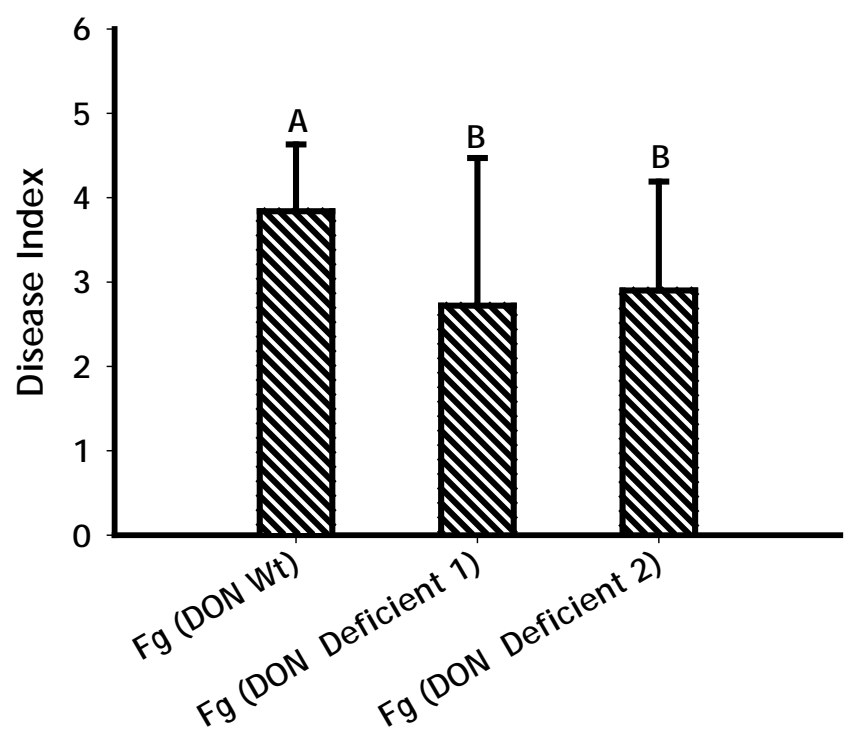

Figure 1. Disease severity rating on wheat (cv. Apogee) infected with F. graminearum. (A) F. graminearum NIV (Tri5+) and NIV (Tri5-) inoculated seedlings. (B) F. graminearum DON (Tri5t) and DON (Tri5-) inoculated seedlings. Bars capped with the same letter are not significantly different based on Kruskal-Wallis test at $P \leq 0.05$. Each bar is the collective mean of three experiments of 50 replicates with (SD).

The NIV (Tri5t) inoculated seedlings suffered severe aggressiveness and resulted in reduced biomass indexes in shoot and root (Fig: 2a). Due to intensified virulence soon after emergence, seedling biomass accounted for on average 14.5 and $12.5 \%$ as a collective mean of fresh and dry biomass of shoot and root (Fig. 3a [A-B]) in comparison to the water controls. NIV (Tri5-) inoculated seedlings however contained adequate amounts of biomass in both 58 and $53.5 \%$ in shoot and 47 and $45.5 \%$ in collective root indexes compared with the controls. Moreover, DON 
(Tri5t) infected seedlings showed greater disease indexes and suffered large losses in different plant parts. The inoculated seedlings with DON (Tri5+) contained on average only 3.5 and $3.75 \%$ collective biomass of fresh and dry shoot and root indexes compared to the water controls (Fig. 2b). Whereas, adequate proportions of biomass was measured in DON (Tri5-) with an average of 31.5 and $15 \%$ as collective shoot and 33.5 and $13 \%$ as collective root biomass compared to the controls. Both NIV (Tri5t) and DON (Tri5t) infected seedlings were tested significantly different $(\mathrm{P}<0.05)$ in comparison to NIV (Tri5-) and DON (Tri5-) infected ones (Fig. 3b). Water inoculated seedlings showed good amounts of biomass, since no pathogen presence was observed. 
Wheat (cv. Apogee) seedling infection with NIV and DON chemotypes of $\mathbf{F}$. graminearum

(a)

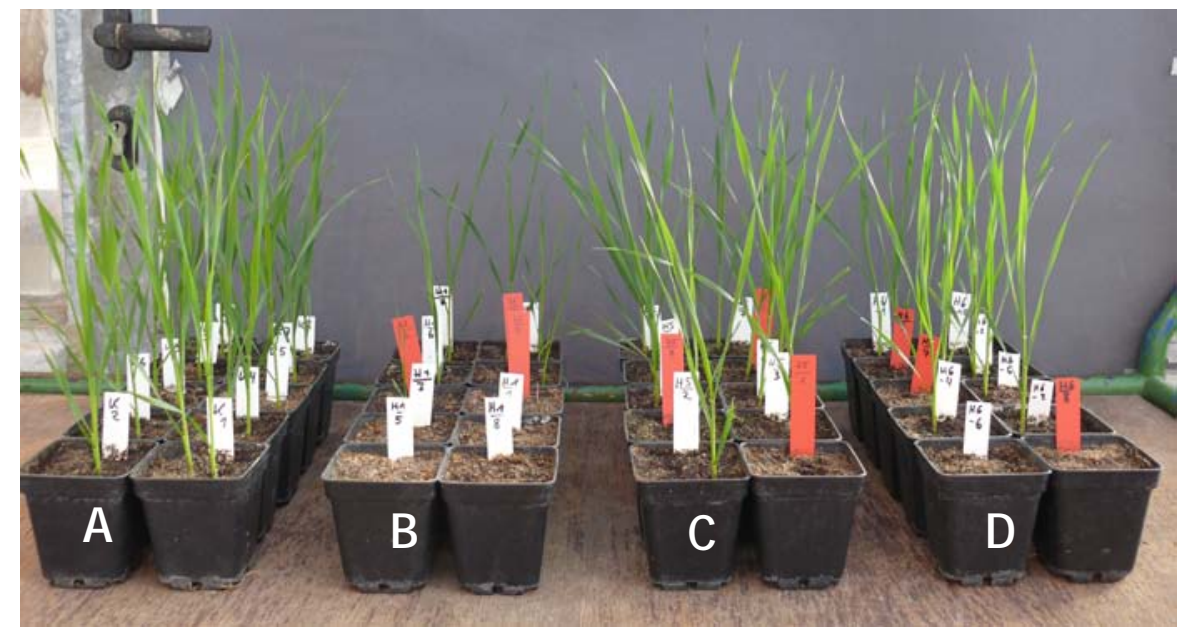

Figure 2(a). Role of NIV chemotype on 4-week-old wheat (cv. Apogee) seedlings. A) Water control, (B) NIV (Tri5+), (C) NIV (Tri5-) and (D) NIV (Tri5-)

(b)

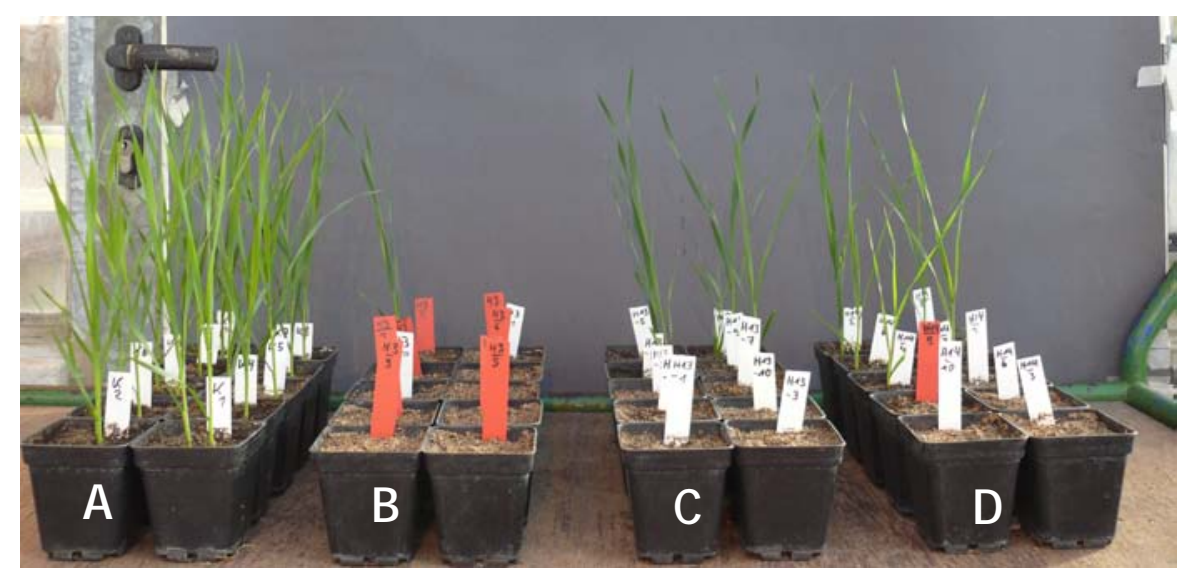

Figure 2(b). Role of DON chemotype on 4-week-old wheat (cv. Apogee) seedlings. A) Water control, (B) DON (Tri5+), (C) DON (Tri5-) and (D) DON (Tri5-) 
(A)

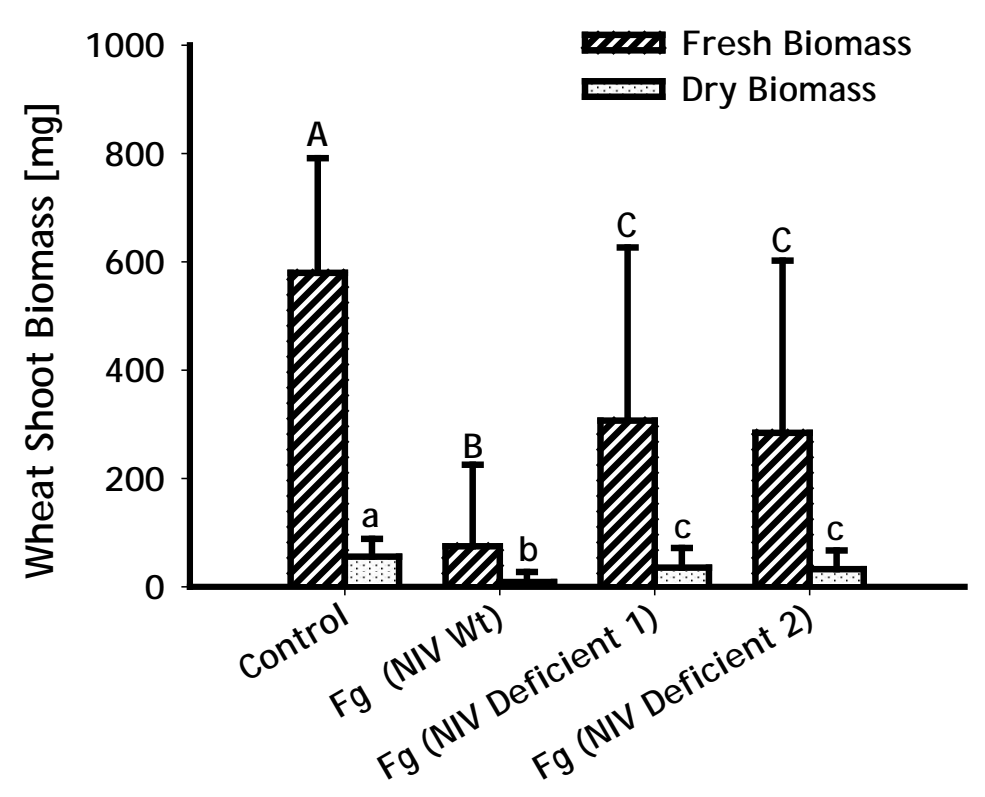

(B)

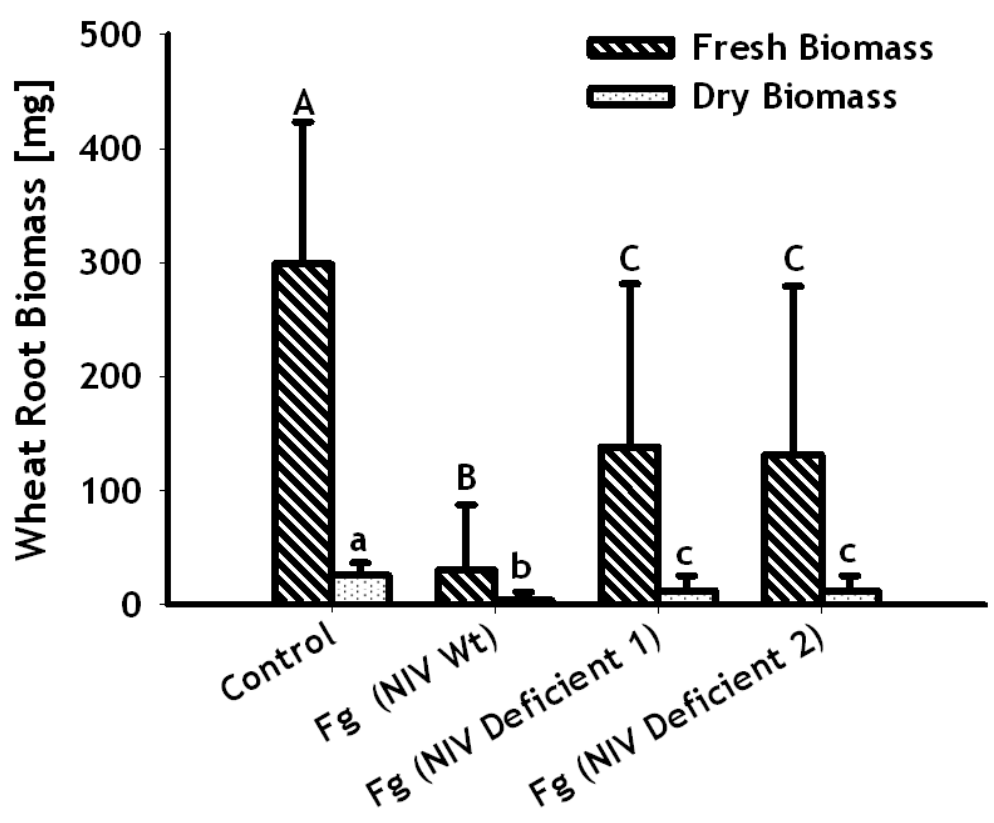

Figure 3(a). Effect of NIV chemotype on wheat (cV. Apogee) seedling biomass. A) shoot indexes, B) root indexes. Bars capped with the same letter are not significantly different based on Kruskal-Wallis test at $P \leq 0.05$. Each bar is the collective mean of three experiments of 50 replicates with (SD). 
(A)

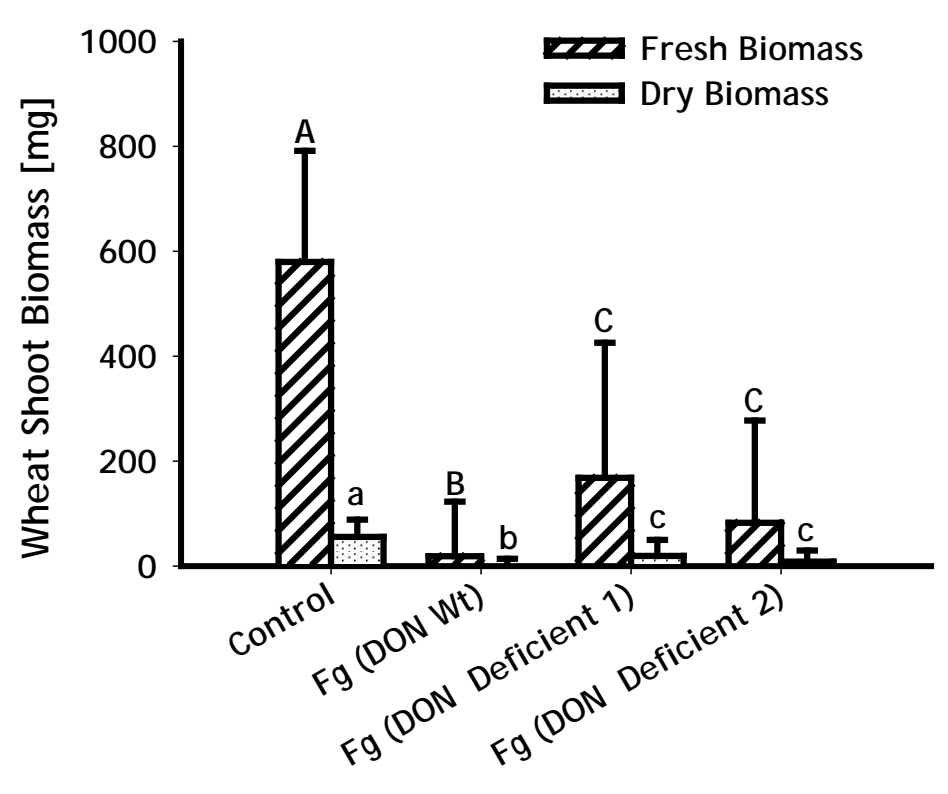

(B)

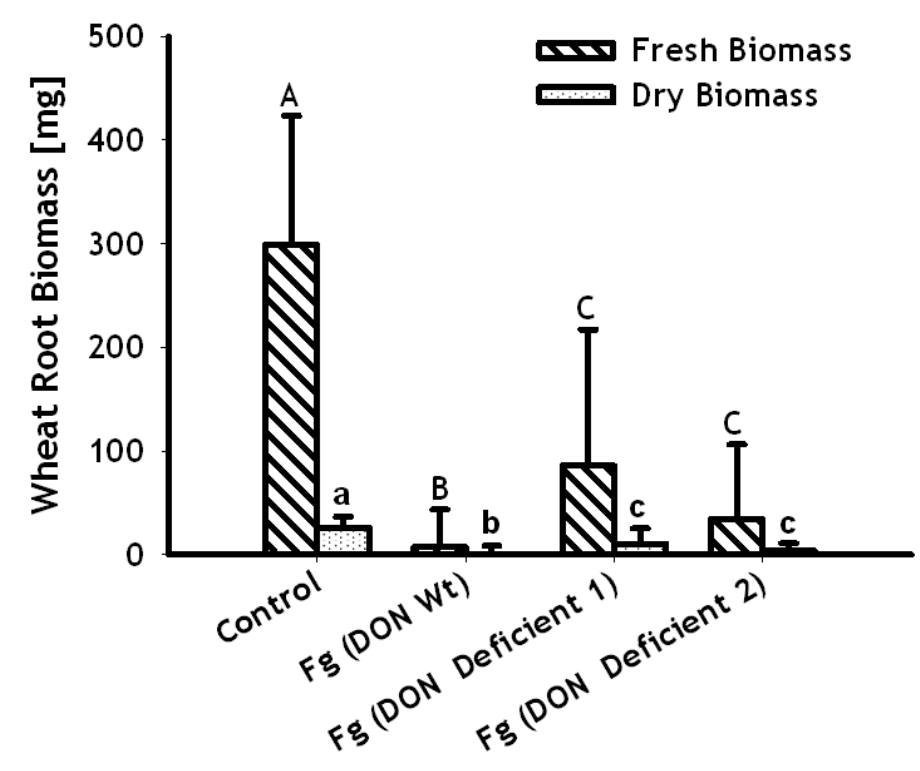

Figure 3(b). Effect of DON chemotype on wheat (cv. Apogee) seedling biomass. A) shoot indexes, B) root indexes. Bars capped with the same letter are not significantly different based on Kruskal-Wallis test at $\mathrm{P} \leq \mathbf{0 . 0 5}$. Each bar is collective mean of three experiments of 50 replicates with (SD).

In the NIV (Tri5t) infected samples, in both roots and shoots fungal DNA was detected as an indicator of fungal biomass. However, traces of DNA were also 
measured in NIV (Tri5-) infected seedlings. The disease score is one of the precursors which witnessed the fungal presence in seedling tissue. Therefore it is explicable that NIV (Tri5+) infected seedlings contained larger fungal biomass than samples infected with NIV (Tri5-). Whereas, the disease severity of NIV (Tri5-) infected seedlings was distinctly lower on disease scale and resulted in small amounts of fungal biomass (Fig. 4).

Moreover, greater proportions of pathogen DNA quantified in root than shoot regardless of NIV (Tri5+) or NIV (Tri5-) infected seedlings. Additionally, fungal DNA was not quantified in either DON (Tri5t) nor in DON (Tri5-) infected samples. Because of the high heterogeneity of the samples, most of the samples were dead seeds or not enough DNA was extracted. Positive-detected samples showed only traces of the fungal presence, the signals were below the detection limit (data not shown).

DON was not quantified in both root and shoot infected DON (Tri5t). Therefore, only traces of NIV was detected in several samples measured in different plant parts infected with NIV (Tri5+) or below the detection limits. 
(A)

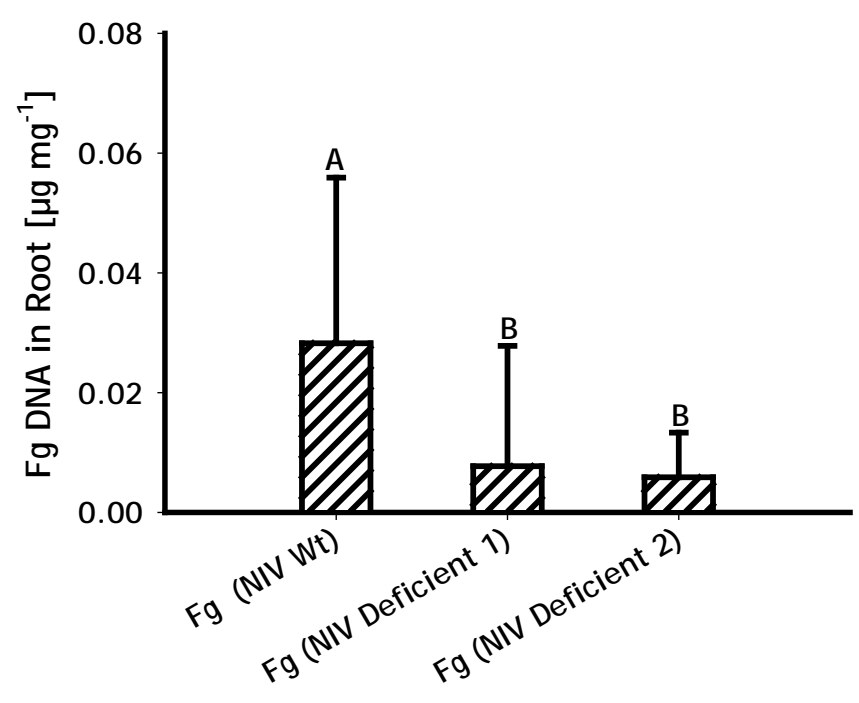

(B)

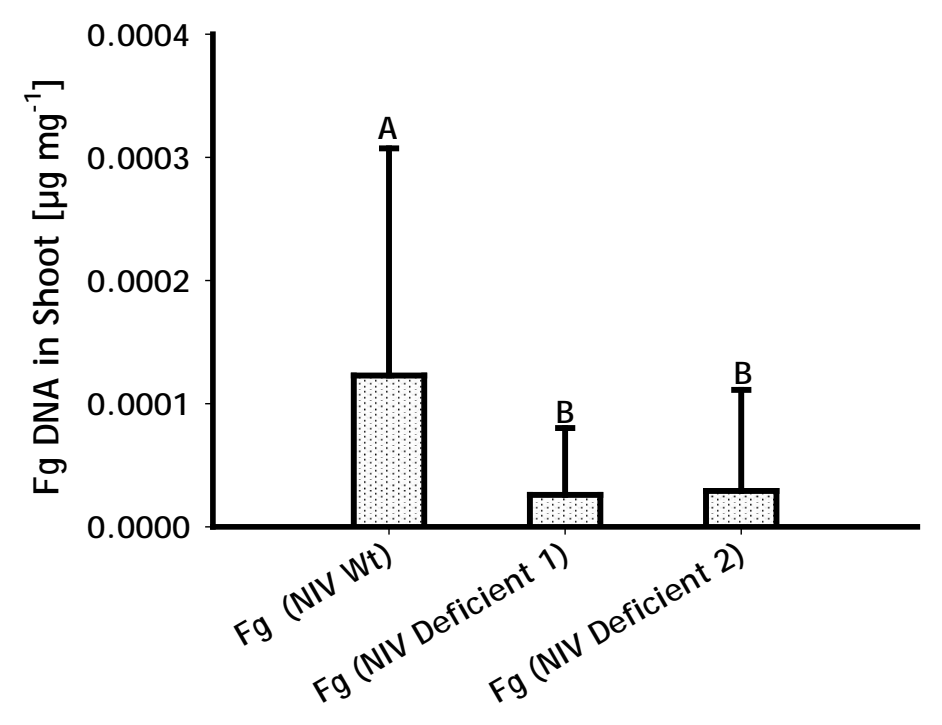

Figure 4. Real- time PCR quantification of F.graminearum DNA in wheat A) DNA in root indexes, B) DNA in shoot indexes. Bars capped with the same letter are not significantly different based on Kruskal-Wallis test at $P \leq 0.05$. Each bar is the mean of three experiments of 50 replicates with (SD).

\section{F. graminearum aggressiveness on maize (cv. Gaspe flint) root}

Both NIV (Tri5t) and DON (Tri5t) were found to be aggressive on maize (cv. Gaspe flint). However, the deficient mutants NIV (Tri5-) and DON (Tri5-) were also able to produce a moderate level of disease (Fig: 5a). The seedling emergence was slightly better in NIV (Tri5-) and DON (Tri5-) but most of the seeds failed to germinate or 
were killed due to the pathogenicity of pathogen before germination (Fig: $5 b$ ). The more severe disease was scored on NIV (Tri5t) and DON (Tri5t) infected seedlings and the same pattern was also observed on NIV (Tri5-) and DON (Tri5-) inoculated samples. No significant differences were observed among different chemotypes (data not shown).

The NIV (Tri5t) virulence was consistent on maize seedlings, however NIV (Tri5-) infected seedlings were not equally affected in both indexes. Equal amount of biomass was measured in NIV (Tri5+) and NIV (Tri5-) in both shoot and root indexes. The mean of the shoot and root biomass was obtained in NIV (Tri5t) inoculated seedlings, which ranged between 38 and $57.5 \%$ against 30 and $43.5 \%$ in shoot and 49 and 62.5 in root NIV (Tri5-) infected indexes. No significant differences $(P<0.05)$ in seedling biomass were obtained after inoculation with NIV (Tri5t) and NIV (Tri5-) mutants (Fig. 6a-b). The DON (Tri5t) produced moderately greater virulence at germination stage and severe symptoms were observed through out whole experiment (Fig. 4b). However, DON (Tri5-) was also able to produce infection to an equal extent but the disease progression remained stable until harvest. The maize seedlings were affected and consequently contained reduced biomass indexes both in root and shoot regardless of chemotype specificity. DON (Tri5t) infected seedlings were ranged at 18.5 and $56 \%$ as collective biomass of shoot and root compared to the control. Whereas, DON (Tri5) $s^{\prime}$ infected seedlings suffered heavy losses and offered 14 and $21.5 \%$ as shoot and 45 and $61 \%$ as root fresh and dry biomass. No significant differences were found among different chemotypes. 


\section{Mini maize (cv.Gaspe flint) seedling infection with NIV and DON chemotypes F. graminearum}

(a)

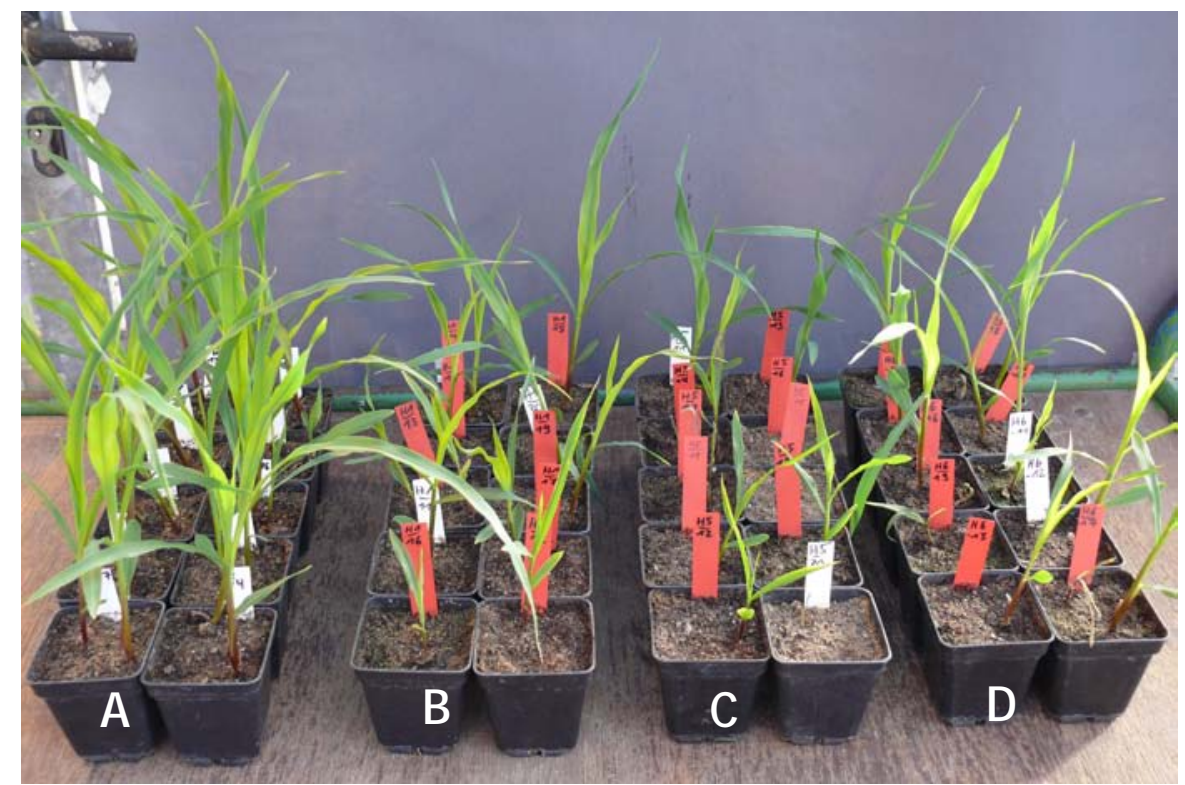

Figure 5(a). Role of NIV chemotype on 4-week-old maize (cv. Gaspe flint) seedlings. A) Water control, (B) NIV (Tri5+) inoculated, (C) NIV (Tri5-) inoculated and (D) NIV (Tri5-) inoculated.

(b)

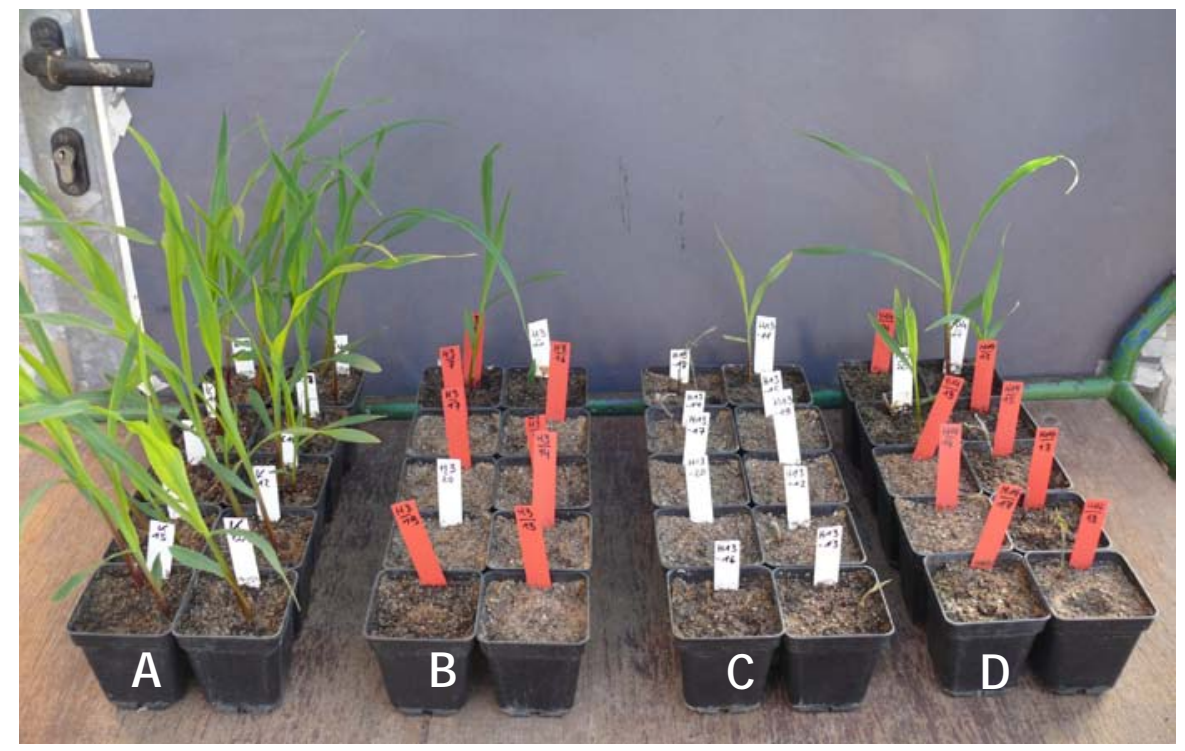

Figure 5(b). Role of DON chemotype on 4-week-old maize (cv. Gaspe flint) seedlings. A) Water control, (B) DON (Tri5+) inoculated, (C) DON (Tri5-) inoculated and (D) DON (Tri5-) inoculated. 
(A)

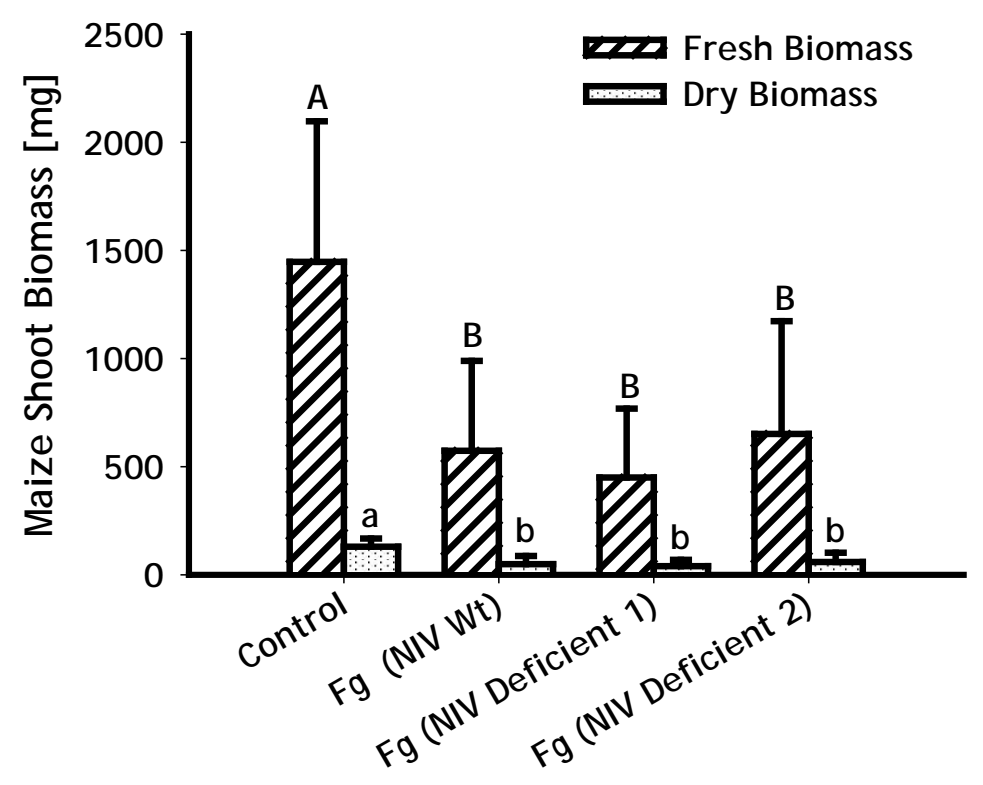

(B)

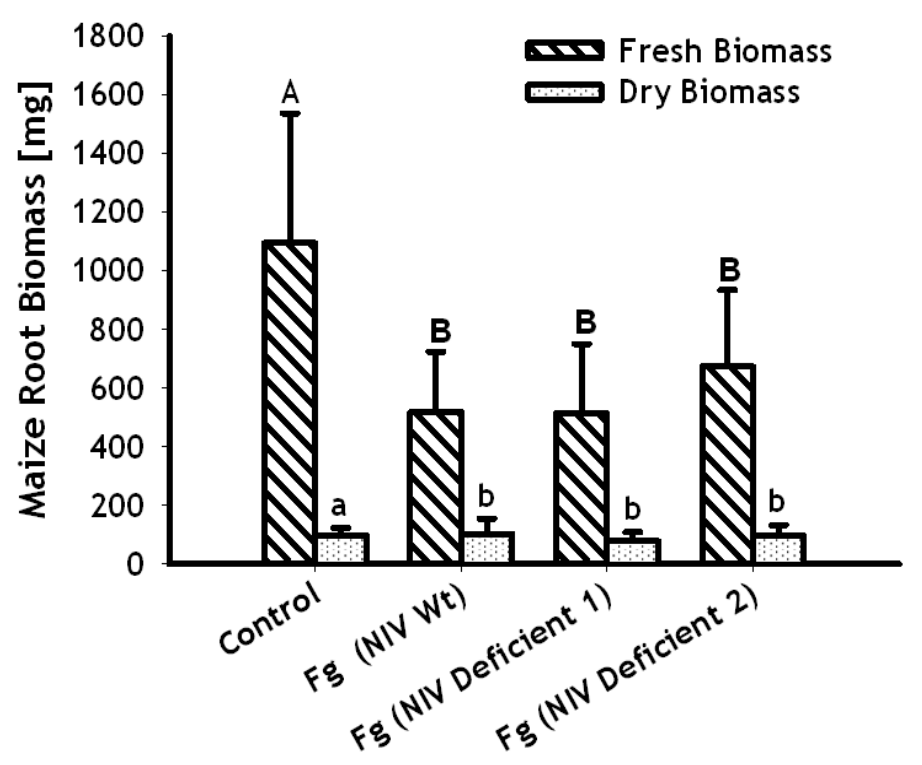

Figure 6(a). Effect of NIV chemotype on maize (cv. Gaspe flint) seedling biomass. A) shoots indexes, B) root indexes. Bars capped with the same letter are not significantly different based on Kruskal-Wallis test at $\mathrm{P} \leq 0 \cdot 05$. Each bar is the collective mean of three experiments of 50 replicates with (SD). 
(A)

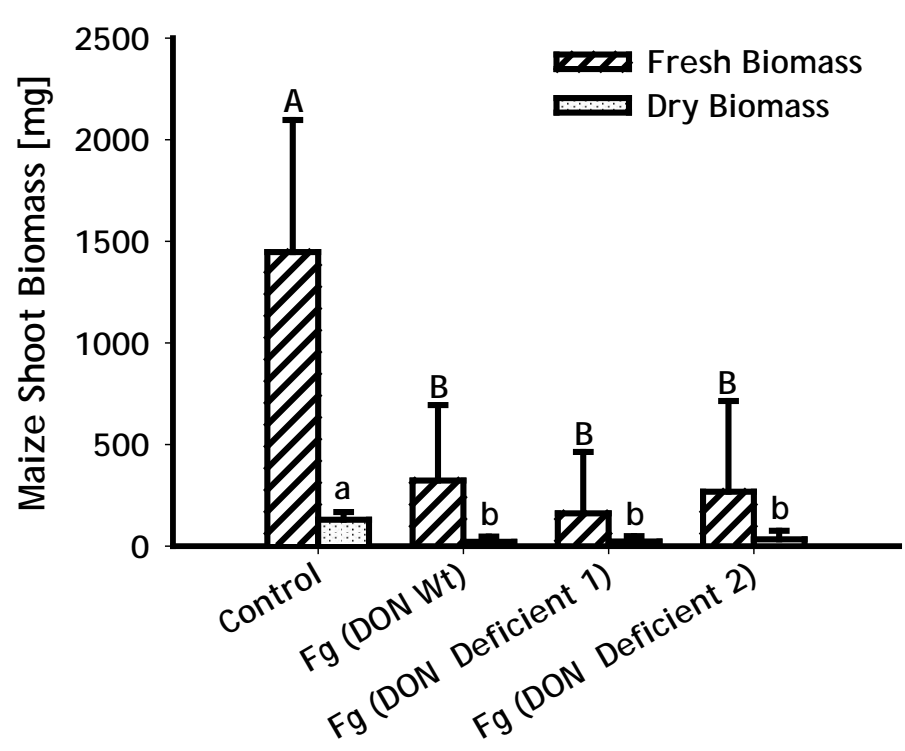

(B)

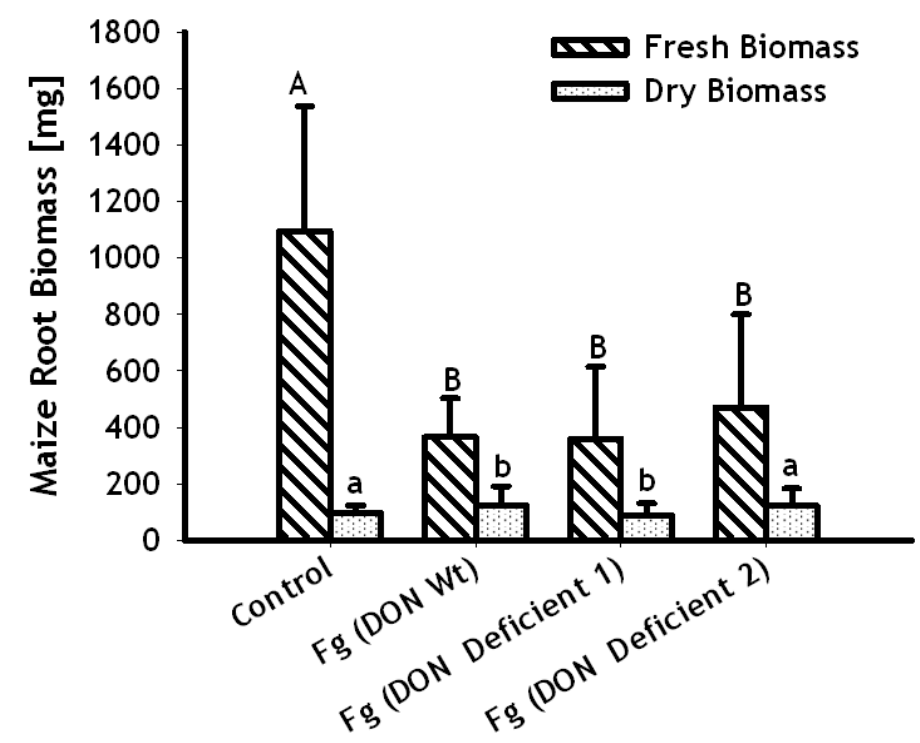

Figure 6(b). Effect of DON chemotype on maize (cv. Gaspe flint) seedling biomass. A) shoots indexes, B) root indexes. Bars capped with the same letter are not significantly different based on Kruskal-Wallis test at $\mathrm{P} \leq 0 \cdot 05$. Each bar is the collective mean of three experiments of 50 replicates with (SD). 


\section{Discussion}

\section{F. graminearum aggressiveness on wheat (cv. Apogee) root}

The trichothecenes DON and NIV are decisive factors for F. graminearum. Their crucial contribution to virulence has been tested on several crops such as wheat and maize ear infection (Bai et al., 2002; Harris et al., 1999; Maier et al., 2006). Several lines of evidence are reported regarding the possible role of NIV in plant pathogenesis. In present studies the NIV (Tri5t) inoculated seeds suffered extreme losses at the germination stage and consequently scored for higher disease severity compared to NIV (Tri5-). NIV is conducive for plant pathogenesis and gives us reason to believe that the NIV concentrations produced in the wheat seedlings were at least partly responsible for the observed disease symptoms. Some earlier reports described that NIV is ten-fold more toxic than DON and could also be more phytotoxic than DON at lower concentrations (Ryu et al., 1988). Our results are in agreement with Maier et al. (2006) that tested the F. graminearum NIV (Tri5-) on maize cobs and found them less virulent. In another study on genetic variations of F. graminearum chemotype, Carter et al. (2002) reported F. graminearum NIV producers were classified to be more aggressive than DON producers on wheat and maize seedlings infection.

In DON (Tri5t) inoculated seeds the seedling emergence turned out to be very low and severe disease was scored. However, moderate disease levels were rated on DON (Tri5-) inoculated plants. In DON (Tri5t) infected seeds most of the disease scored on the dead seeds because they received severe infection. Similar results were reported from (Chongo et al., 2001) whose disease score was completely based on dead seeds because of severe infection in wheat seedlings. One reason for the number of dead seeds before emergence could be the high toxicity of DON against wheat. Moreover, even lower concentrations of DON were at least partly responsible for severe disease symptoms. The DON phytotoxicity has been documented in some studies (Bruins et al., 1993; Eudes et al., 2000; Shimada and Otani, 1990). In vitro cultures, concentrations up to $2 \mu \mathrm{g} / \mathrm{ml}$ severely inhibited coleoptile's elongation of wheat seedlings and at $100 \mu \mathrm{g} / \mathrm{ml}$ germination ceased (Bandurska et al., 1994). Our results are in accordance with Wang et al. (2006) who 
tested F. graminearum DON (Tri5-) on wheat, barley and triticale root infection and reported that DON (Tri5-) was less aggressive than progenitor strains.

The seedlings inoculated with NIV (Tri5t) reduced plant biomass drastically; however large quantities were weighed in NIV (Tri5-) inoculations (Fig. 2a). The average biomass in shoot and root indexes remained one-fold in NIV (Tri5+), 5-fold biomass increase was measured in NIV (Tri5-) infected plants compared to the water controls (Fig. 3a). Similar findings were obtained from Carter and co-worker (2002) who observed reduced biomass in infected seedlings with NIV chemotype. DON (Tri5+) inoculated seedlings remained on one-fold measured biomass, and 10fold increase in biomass was measured in shoot and root indexes of DON (Tri5-) in comparison to the water controls (Fig. 3b). Our obtained results validate Deshmukh and Kogel (2007) reporting reduced barley seedlings biomass after F. graminearum infections.

Large amount of DNA content was quantified on NIV (Tri5+) inoculated seedlings. Therefore, more DNA was measured in infected root than shoot indexes. The total DNA contents in NIV (Tri5t) infected samples were many fold greater than with NIV deficient mutants infected ones (Fig. 4). The disease symptoms were more severe on roots than shoots, which indicates heavy fungal biomass accumulations. Moreover, NIV (Tri5-) infected samples were also positively measured for DNA contents but only little fungal biomass was detected. One reason of lower DNA detection in root or shoot could be that NIV aids pathogen in disease progression and colonization of the seedling tissues in root and shoot. Unfortunately, large heterogeneity was observed in DON (Tri5+) infected samples, most of the samples consisted of dead seeds or blighted seedlings. The plant material was not enough for DNA extraction. Our results are in support of (Deshmukh and Kogel, 2007) who detected F. graminearum in infected barley roots, so it could be expected that fungal DNA was also present at least in the roots of the DON (Tri5t) infected samples.

Neither NIV nor DON could be quantified in root and shoot infected with NIV (Tri5t) or DON (Tri5+). There were only traces of NIV detected and several measured samples were below the detection limit. Our findings are in contrast to the results of (Asran and Buchenauer, 2003) who determined the DON contents and ergosterol concentrations in corn seedling tissue in an aeroponic system after infection with F. graminearum. It could be suggested that the contribution of NIV or DON in plant pathogenesis may diminish, if the conditions for disease development improves. A 
second explanation could be the seedling progression. It is conceivable that the NIV or DON concentrations were diluted with the ongoing growth of the seedlings, and were therefore not detectable. However, this could only be the case, if the DON and NIV production is restricted to the beginning of the pathogenesis. In our studied system seed serves as a small nutrient reservoir for the pathogen. The small concentrations of these trichothecenes could be enough for the pathogenicity. In ecological settings, NIV or DON are in abundance where large sinks of nutrient available such as wheat ears and maize cobs.

\section{F. graminearum aggressiveness on maize (cv. Gaspe flint) root}

The maize cv. Gaspe flint seed inoculation with either NIV (Tri5t) or DON (Tri5t) resulted in poor emergence. However, emergence was better in NIV (Tri5-) or DON (Tri5-) inoculated seeds (Fig. 5a). There were no significant differences detected for the disease severity among (Tri5t)s' and (Tri5-)s' chemotypes. Mostly dead seeds were surface sterilized and placed on PDA. Afterwards mainly dead seeds were confirmed to contain large mycelia on re-isolation. The disease index was slightly lower on NIV or DON (Tri5-) infected seedlings than on the wild type infected ones (data not shown). No significant differences were observed in the disease indexes of both NIV (Tri5+) and DON (Tri5t) infected seedlings, compared with the ones inoculated with their respective mutants. The same was true for the root and shoot biomass of both NIV (Tri5t) and DON (Tri5t) infected seedlings, compared with the plants infected with the respective knock-out mutants (Fig. 5b +6b). Our results supported with (Proctor et al., 1995) who observed DON (Tri5-) strains as aggressive as progenitors on Golden Bantam maize.

In our conducted studies, NIV and DON are confirmed as virulence factors on wheat root infection. The deficient mutants were also able to produce infection at emergence stage, but were not found to be as aggressive as the wild-types. The larger amount of fungal biomass in NIV (Tri5+) wheat roots or shoots is another witness to validate these findings. On maize NIV (Tri5-) or DON (Tri5-) were recorded equally aggressive as their wild-type counterparts. The disease severity remained at a similar level on maize seedlings, regardless of wild-type infected or deficient mutants. Nevertheless, DON infected wheat seedlings showed more severe symptoms than NIV but in our studies we were not able to quantify either 
DON or NIV out of wheat or maize. So it may be suggested that in the infected plants the amount of DON produced was more than NIV. Several researchers have concluded that in maize cob infection the disease severity was equal for both NIV and DON chemotypes. However, the infected cobs contained more DON accumulation than NIV.

\section{References}

1. Asran, M. R. and H. Buchenauer. 2003. Pathogenicity of Fusarium graminearum isolates on maize (Zea mays L.) cultivars and relation with deoxynivalenol and ergosterol contents. Zeitschrift fur Pflanzenkrankheiten und Pflanzenschutz-J ournal of Plant Diseases and Protection 110:209-219.

2. Bai, G. H., A. E. Desjardins, and R. D. Plattner. 2002. Deoxynivalenolnonproducing Fusarium graminearum causes initial infection, but does not cause disease spread in wheat spikes. Mycopathologia 153:91-98.

3. Bai, G. H. and G. Shaner. 1996. Variation in Fusarium graminearum and cultivar resistance to wheat scab. Plant Dis. 80:975-979.

4. Bandurska, H., J. Chelkowski, and H. Wisniewska. 1994. Free Proline Accumulation in Wheat Seedlings Influenced by Fusarium culmorum Infection and the Pathogen Metabolite Deoxynivalenol. Acta Physiologiae Plantarum 16:111-116.

5. Brandfass, C. and P. Karlovsky. 2008. Upscaled CTAB-based DNA extraction and real-time PCR assays for Fusarium culmorum and F. graminearum DNA in plant material with reduced sampling error. International Journal of Molecular Sciences 9:2306-2321.

6. Bruins, M. B. M., I. Karsai, J. Schepers, and C. H. A. Snijders. 1993. Phytotoxicity of deoxynivalenol to wheat tissue with regard to in vitro selection for Fusarium head blight resistance. Plant Science 94:195-206.

7. Bugbee, B., G. Koerner, R. Albrechtsen, W. Dewey, and S. Clawson. 1997. Registration of 'USU-Apogee' wheat. Crop Science 37:626.

8. Carter, J. P., H. N. Rezanoor, D. Holden, A. E. Desjardins, R. D. Plattner, and P. Nicholson. 2002. Variation in pathogenicity associated with the genetic diversity of Fusarium graminearum. European Journal of Plant Pathology 108:573-583.

9. Chongo, G., B. D. Gossen, H. R. Kutcher, J. Gilbert, T. K. Turkington, M. R. Fernandez, and D. McLaren. 2001. Reaction of seedling roots of 14 crop species to Fusarium graminearum from wheat heads. Canadian J ournal of Plant Pathology-Revue Canadienne de Phytopathologie 23:132-137. 
10. Deshmukh, S. D. and K. H. Kogel. 2007. Piriformospora indica protects barley from root rot caused by Fusarium graminearum. Journal of Plant Diseases and Protection 114:263-268.

11. Desjardins, A. E. and T. M. Hohn. 1997. Mycotoxins in plant pathogenesis. Molecular Plant-Microbe Interactions 10:147-152.

12. Eudes, F., A. Comeau, S. Rioux, and J. Collin. 2000. Phytotoxicity of eight mycotoxins associated with fusarium in wheat head blight. Canadian J ournal of Plant Pathology-Revue Canadienne de Phytopathologie 22:286-292.

13. Gilbert, J. and W. G. D. Fernando. 2004. Epidemiology and biological control of Gibberella zeae Fusarium graminearum. Canadian Journal of Plant Pathology-Revue Canadienne de Phytopathologie 26:464-472.

14. Harris, L. J., A. E. Desjardins, R. D. Plattner, P. Nicholson, G. Butler, J. C. Young, G. Weston, R. H. Proctor, and T. M. Hohn. 1999. Possible role of trichothecene mycotoxins in virulence of Fusarium graminearum on maize. Plant Disease 83:954-960.

15. J ennings, P., M. E. Coates, K. Walsh, J. A. Turner, and P. Nicholson. 2004. Determination of deoxynivalenol- and nivalenol-producing chemotypes of Fusarium graminearum isolated from wheat crops in England and Wales. Plant Pathology 53:643-652.

16. Kimura, M., I. Kaneko, M. Komiyama, A. Takatsuki, H. Koshino, K. Yoneyama, and I. Yamaguchi. 1998. Trichothecene 3-0-acetyltransferase protects both the producing organism and transformed yeast from related mycotoxins - Cloning and characterization of Tri101. J ournal of Biological Chemistry 273: 1654-1661.

17. Knudsen, I. M. B., J. Hockenhull, and D. F. Jensen. 1995. Biocontrol of seedling diseases of barley and wheat caused by Fusarium culmorum and Bipolaris sorokiniana - Effects of selected fungal antagonists on growth and yield components. Plant Pathology 44:467-477.

18. Ludewig, A., U. Kabsch, and J. A. Verreet. 2005. Mycotoxin production of 31 Fusarium graminearum isolates and aggressiveness against wheat seedlings. Zeitschrift fur Pflanzenkrankheiten und Pflanzenschutz-J ournal of Plant Diseases and Protection 112: 124-133.

19. Maier, F. J., T. Miedaner, B. Hadeler, A. Felk, S. Salomon, M. Lemmens, H. Kassner, and W. Schafer. 2006. Involvement of trichothecenes in fusarioses of wheat, barley and maize evaluated by gene disruption of the trichodiene synthase (Tri5) gene in three field isolates of different chemotype and virulence. Mol. Plant Pathol. 7:449-461.

20. Miedaner, T., C. Reinbrecht, and A. G. Schilling. 2000. Association among aggressiveness, fungal colonization, and mycotoxin production of 26 isolates of Fusarium graminearum in winter rye head blight. Zeitschrift fur Pflanzenkrankheiten und Pflanzenschutz-Journal of Plant Diseases and Protection 107: 124-134. 
21. Nganje, W. E., D. A. Bangsund, F. L. Leistritz, W. W. Wilson, and N. M. Tiapo. 2004. Regional economic impacts of Fusarium head blight in wheat and barley. Review of Agricultural Economics 26:332-347.

22. Nicholson, P., D. R. Simpson, G. Weston, H. N. Rezanoor, A. K. Lees, D. W. Parry, and D. Joyce. 1998. Detection and quantification of Fusarium culmorum and Fusarium graminearum in cereals using PCR assays. Physiological and Molecular Plant Pathology 53:17-37.

23. Parry, D. W., P. Jenkinson, and L. Mcleod. 1995. Fusarium ear blight (scab) in small grain cereals - A review. Plant Pathology 44:207-238.

24. Proctor, R. H., T. M. Hohn, and S. P. McCormick. 1995. Reduced virulence of Gibberella zeae caused by disruption of a trichothecene toxin biosynthetic gene. Mol.Plant Microbe Interact. 8:593-601.

25. Ryu, J. C., K. Ohtsubo, N. Izumiyama, K. Nakamura, T. Tanaka, H. Yamamura, and Y. Ueno. 1988. The acute and chronic toxicities of nivalenol in mice. Fundamental and Applied Toxicology 11:38-47.

26. Shimada, T. and M. Otani. 1990. Effects of Fusarium mycotoxins on the growth of shoots and roots at germination in some J apanese wheat cultivars. Cereal Research Communications 18:229-232.

27. Snijders, C. H. A. 1990. Fusarium head blight and mycotoxin contamination of wheat- A review. Netherlands J ournal of Plant Pathology 96: 187-198.

28. Sutton, J. C. 1982. Epidemiology of wheat head blight and maize ear rot caused by Fusarium graminearum. Canadian Journal of Plant Pathology 4: 195.

29. Wang, H., S. F. Hwang, F. Eudes, K. F. Chang, R. J. Howard, and G. D. Turnbull. 2006. Trichothecenes and aggressiveness of Fusarium graminearum causing seedling blight and root rot in cereals. Plant Pathology 55:224-230.

30. Ward, T. J., J. P. Bielawski, H. C. Kistler, E. Sullivan, and K. O'Donnell. 2002. Ancestral polymorphism and adaptive evolution in the trichothecene mycotoxin gene cluster of phytopathogenic Fusarium. Proceedings of the National Academy of Sciences of the United States of America 99:9278-9283. 


\section{Chapter 5: General Discussion}

\section{Role of zearalenone in the interaction of $F$. graminearum with G. roseum in vitro and in maize and wheat rhizosphere}

Based on the results we obtained differences between the G. roseum wild-type and the Zes2 mutant. In mixed colony co-incubation, we observed in G. roseum wildtype the colony diameter expanded and pigments scheme changed as incubation timed progressed. This could not be observed in the Zes2 mutant grown on F. graminearum. It can be predicted that a great enzymatic activity took place between the mycoparasite and the pathogen. G. roseum has a very high substrate specificity and can hydrolyze zearalenone (ZEN) into a non-estrogenic product (Elsharkawy and Abulhajj, 1988; Utermark and Karlovsky, 2007; Volkl et al., 1997). We were not able to quantify any ZEN out of the mixed cultures. Our findings are in accordance with previous results of Utermark and Karlovsky (2007) who observed a growth retardation of Zes2 mutants of $G$. roseum on ZEN $20 \mu \mathrm{g} / \mathrm{ml}$ amended Czapek-Dox-medium, therefore no inhibition was observed in G. roseum wild-type. Moreover, the inhibitory effects were also found on ten filamentous fungi grown on plates containing low ZEN concentration $(2 \mu \mathrm{g} / \mathrm{ml})$ and complete inhibition was measured at $\geq 10 \mu \mathrm{g} / \mathrm{ml}$ with an exception of $\mathrm{G}$. roseum on Malt-Extract-Agarmedium (Utermark and Karlovsky, 2007).

In greenhouse trials we used 5-pre-screened aggressive strains of F.graminearum to infect maize and wheat seeds. On big reason to use several strains was to test the differences between $\mathrm{G}$. roseum wild-type and Zes2 mutants on co-inoculation with pathogen and to validate the results credibility. We found a great homogeneity in seed or seedling infection with all tested strains. When we co-inoculated the strains with either $G$. roseum wild-type or Zes2 mutant, we observed significant differences. The wild-type inoculated seedlings of either maize or wheat showed more protection, improved seed germination, good yield accumulation in root and shoot indexes and less disease severity compared to the Zes2 mutant.

These results give strong evidence that ZEN is not involved in pathogenicity but rather in substrate colonization. The percentage of dead or diseased seeds was relatively greater in Zes2 co-inoculated plants rather than in wild-type inoculated 
plants. The improved seedlings emergence and greater biomass indexes in $\mathrm{G}$. roseum wild-type validates the fast colonization of the mycoparasite, however Zes2 inhibited somehow against pathogen and partially protected the seedlings. In previous findings Gaffoor et al. (2005) reported that ZEN does not play any role in plant pathogenesis on wheat heads when no significant differences observed between F.graminearum wild-type and ZEN deficient mutants and both behaved in similar manner. Therefore, ZEN helps its producer (F.graminearum) in grains colonization, protection of substrate and adverse survival conditions (Gaffoor et al., 2005; Kim et al., 2005). The gene expression studies revealed that ZEA1 and ZEA2,( gene responsible for ZEN biosynthesis in F. graminearum)(Gaffoor et al., 2005), are up regulated in grain colonization bioassays (Gaffoor et al., 2005). ZEN is normally produced later than DON or NIV while both are believed to occur early in the plant or seed infection (Goswami and Kistler, 2004; Munkvold, 2003; Yoshida and Nakajima, 2010). However, ZEN is never reported to be involved in pathogenesis (Gaffoor et al., 2005) but its late production in maize fields or in stored grains are widely documented (Martins and Martins, 2002; Sutton, 1982).

The gene specific to ZEN-lactonase enzyme of $G$. roseum was previously cloned in different groups (Kakeya et al., 2002; Takahashi-Ando et al., 2002) but the specific role of ZEN-lactonase of $\mathrm{G}$. roseum and gene-knock-out mutants were generated and tested on ZEN by (Utermark and Karlovsky, 2007). However, the genetic transformation of gene (ZEN-lactonase) was done in a maize hybrid line (Igawa et al., 2007). The ecological role of ZEN was never tested before in the rhizoshpere root colonization of maize and wheat infected by $F$. graminearum. However, several other researchers have used $G$. roseum as a promising biological control agent. Vakili (1992) reported that G. roseum suppressed the Fusarium moniliforme growth on corn in vitro and in field trials. Knudsen (1994) observed great effects of the mycoparasite against B. sorokiniana in infected seedlings and isolated the endophyte from distal and proximal root segments. Burgess et al. (1997) tested the impact of the mycoparasite against Botrytis cinerea on chickpea. Xue (2003) investigated Clonostachys rosea on field pea root rot disease and recorded vast disease suppression. The rhizosphere colonization of barley and wheat seedlings with the mycoparasite G. roseum against F. culmorum was investigated by few researchers (J ensen et al., 2000; Knudsen et al., 1995) and reported to cause a significant disease suppression in root rot diseases. The focus of all these studies 
was mostly to screen the biocontrol agent or to investigate the antagonistic potential of mycoparasitic fungi against several seed and soil-borne pathogens.

We estimate the role of ZEN in ecological settings, potential reason for its production and the biological significance to its producer. G. roseum has huge substrate specificity and ZEN severed as a precursor for the active release of the ZEN-lactonase enzyme of $G$. roesum.

The role of $F$. graminearum as the most associated pathogen of head blight in wheat and barley and ear rot in maize has been extensively reviewed (Goswami and Kistler, 2004; Logrieco et al., 2002; Munkvold, 2003; Parry et al., 1995; Sutton, 1982). However, its pathogenicity in seedling infection has not been thoroughly investigated. The root rots or seedling blights are not considered as substantial economic threats, but the inoculum production and saprophytic phase of the pathogen can not be neglected. There are few studies conducted on the role of $F$. graminearum on wheat or maize seedlings. The objective of most of the studies was to understand the role of trichothecenes in the pathogenicity or aggressiveness to the pathogen. Our findings may conclude that ZEN is initially involved in substrate colonization of the seeds and some of the trichothecenes are primarily involved in disease initiation or initial infection. It is noticed that most of the seedling- disease infection- bioassays do not properly quantify DON or NIV or any other trichothecenes. There are various studies conducted to investigate the role of DON and NIV on the roots of seedlings or on seed germination, but to our knowledge most of the studies were conducted under sterile or axenic conditions, or synthetic toxins were used to treat the seeds or seedlings. Future studies should be focused on the quantification of ZEN from root samples or other plant parts coinoculated with F. graminearum and G. roseum. This would further concrete the findings concerning the ecological role of ZEN in root colonization.

\section{Pathogenicity and mycotoxin production of different F.graminearum strains on maize and wheat ear and root infection}

We have highlighted and synthesized several different factors which are extremely important in determining the role of $\mathrm{F}$. graminearum in wheat and maize infection. 
F. graminearum infection or pathogenicity depends on numerous factors which contribute directly or indirectly to the Fusarium-caused epidemics. According to our findings, trichothecenes (DON and NIV) seem to be the most important determinants of disease concerning head blight in wheat and ear rot in maize. We found an identical pattern of virulence on both wheat and maize ear and root infection. Our observations in wheat and maize after root infection lead to the outcome that DON and NIV have inhibitory effects on seedlings roots, and their role is largely involved in disease virulence. Most of the seedlings in maize and wheat died after germination with acute blight symptoms which clearly formulate a line of evidence that there are some other toxic metabolites contribute to initiate the infection too. However, DON and NIV may be biosynthesized during the infection process besides other metabolites. It is well-documented in literature that DON and NIV have inhibition effects on seed germination and coleoptile elongation in wheat (Eudes et al., 2000; Shimada and Otani, 1990). However, several non-toxigenic fusion products are found to be highly pathogenic on maize ears and carnation flowers (Adams and Hart, 1989). We observed the great magnitude of disease on wheat head and maize ears which consequently yielded in large mycotoxin accumulation. The strong positive correlation of strains virulence among maize and wheat ears and roots further elaborate that it is the amount of DON or NIV which is decisive factor for the disease magnitude rather than the chemotype. The DON producers dominated the chemotype in our tested strains but NIV producers also turned out to produce an equal disease severity with reduced NIV accumulation in wheat and maize ears and roots. It is confirmed so far that DON and NIV are virulent factors of F. graminearum in wheat and maize ear infection, but the (Tri5) deficient mutants were also found to be pathogenic but less virulence than the wild-type (Bai et al., 2002; Harris et al., 1999; Maier et al., 2006). The quantification of ZEN in all infected maize ears and partial detection in wheat ears place a serious concern on its usual production. ZEN has been defined as a post harvest problem rather than as a core metabolite for virulence (Gaffoor et al., 2005; Martins and Martins, 2002; Sutton, 1982; Utermark and Karlovsky, 2007). In functional gene-knock-out studies of F. graminearum, ZEN turned out not to be a virulence factor on wheat head (Gaffoor et al., 2005). Moreover, Gaffoor and coworker confirmed that the gene expression of ZEA1 and ZEA2, (which are responsible for ZEN biosynthesis in $\mathrm{F}$. graminearum), was high in grain colonization and substrate protection. However, Kim et al. (2005) proposed that ZEN aids the 
pathogen in adverse survival conditions. In another study, (Utermark and Karlovsky, 2007) reported that ZEN served in interference competition and protected the substrate against its competitors. Our tested correlation between maize and wheat root infection is supplemented by findings of (Carter et al., 2002). The DON contents in wheat and maize correlated with disease severity are endorsed with results of numerous other researchers (Bai et al., 2002; Harris et al., 1999; Maier et al., 2006; Reid et al., 1996), as well as the DNA contents and DON correlation (Demeke et al., 2010; Zhang et al., 2009).

In conclusion, the concrete virulence is found more on maize than wheat. One great reason to explain the phenomena could be the pathogen choice to grow and breed on maize. Therefore, vegetative duration among different crop rotation periods, adaptability as niche, host specificity and population distribution could also be important factors for the pathogen variability. Our results further cements the idea that a large genetic variation exists within strains and populations and the obtained results could place one more step to frame and design different models for trichothecenes distributions, epidemiology and host specificity.

\section{Role of deoxynivalenol (DON) and nivalenol (NIV) in wheat and maize root infection by $F$. graminearum}

Trichothecenes, especially DON and NIV, are of particular importance because of their common accumulation in wheat, barley and maize. DON and NIV are thoroughly investigated in two most common disease Fusarium head blight and Fusarium ear rot which is primarily caused by $\mathrm{F}$. graminearum. Both DON and NIV are primarily involved in virulence on wheat head and maize ears and are reported as virulence factors (Bai et al., 2002; Harris et al., 1999; Maier et al., 2006). Trichothecenes are phytotoxic (Eudes et al., 2000; Shimada and Otani, 1990) and at very low concentrations cause wilting, chlorosis, necrosis, and other symptoms in a wide variety of plants (Cutler, 1988). DON has been reported to be multiplefold more toxic than NIV, T-2 toxin, HT-2 and DAS on wheat seedlings and inhibited coleoptile elongation at very lower concentrations ((Shimada and Otani, 1990). Most of these studies carried out either in vitro or in sterile conditions in axenic cultures using synthetic DON or other toxins. These few studies conducted to 
elucidate the possible role of DON or NIV in root infection on cereals or maize. Carter et al. (2002) reported in genetic variation studies on F. graminearum that NIV producers are more virulent on maize seedlings than DON producers, but large differences were observed in virulence of both DON and NIV chemotypes. Wang et al. (2006) inoculated F. graminearum DON deficient (Tri5-) mutants and wild-type strain (Tri5+) on wheat, barley and triticale seedlings and noticed that DON (Tri5-) strains were less virulent than progenitors. The work considered as little inconsistent while the DON (Tri5-) mutants also posed the same level of virulence on some tested cereals cultivars, (Ning 8331, AC Metcalf, AC Copia). In our conducted work, we took more exclusive approach to target the role of NIV and DON in wheat and maize root infection. We inoculated the seeds of either wheat or maize with two F. graminearum wild-type (Tri5t) strains specific to DON and NIV chemotypes and two individual deficient mutants (Tri5-) correspond to each chemotypes. In wheat inoculated with NIV wild-type, the seeds showed poor emergence, and the seedlings yielded on reduced root and shoot indexes and increased disease severity compared to the deficient mutants. The DNA contents showed a larger amount of F. graminearum- DNA in the NIV wild-type infected roots or shoots than in the deficient mutants, where only traces of fungal DNA were detected. NIV was detected in traceable amounts in the wheat roots or shoots, but with a great variability among samples or below the quantification limit. In maize inoculated with either NIV wild-type or its NIV deficient mutants both produced similar level of disease. DON wild-type inoculated wheat suffered extreme losses in germination, reduced biomass indexes and greater disease severity than the plants infected with the DON deficient mutants. No significant differences were observed between the DON wild-type and deficient mutants inoculated on maize.

We can conclude that NIV and DON are virulent factors of F. graminearum for root infection on wheat, but not on maize.

\section{References}

1. Adams, G. C. and L. P. Hart. 1989. The role of deoxynivalenol and 15acetyldeoxynivalenol in pathogenesis by Gibberella zeae, as elucidated through protoplast fusions between toxigenic and nontoxigenic strains. Phytopathology 79:404-408. 
2. Bai, G. H., A. E. Desjardins, and R. D. Plattner. 2002. Deoxynivalenolnonproducing Fusarium graminearum causes initial infection, but does not cause disease spread in wheat spikes. Mycopathologia 153:91-98.

3. Burgess, D. R., T. Bretag, and P. J. Keane. 1997. Biocontrol of seedborne Botrytis cinerea in chickpea with Gliocladium roseum. Plant Pathology 46:298-305.

4. Carter, J. P., H. N. Rezanoor, D. Holden, A. E. Desjardins, R. D. Plattner, and P. Nicholson. 2002. Variation in pathogenicity associated with the genetic diversity of Fusarium graminearum. European Journal of Plant Pathology 108:573-583.

5. Cutler, H. 1988. Trichothecenes and their role in the expression of plant disease. Biotechnology of Crop Protection, ACD Symposium Series 379:50-72.

6. Demeke, T., T. Graafenhan, R. M. Clear, A. Phan, I. Ratnayaka, J. Chapados, S. K. Patrick, D. Gaba, C. A. Levesque, and K. A. Seifert. 2010. Development of a specific TaqMan (R) real-time PCR assay for quantification of Fusarium graminearum clade 7 and comparison of fungal biomass determined by PCR vvith deoxynivalenol content in wheat and barley. International J ournal of Food Microbiology 141:45-50.

7. Elsharkawy, S. and Y. J. Abulhajj. 1988. Microbial cleavage of zearalenone. Xenobiotica 18:365-371.

8. Eudes, F., A. Comeau, S. Rioux, and J. Collin. 2000. Phytotoxicity of eight mycotoxins associated with fusarium in wheat head blight. Canadian J ournal of Plant Pathology-Revue Canadienne de Phytopathologie 22:286-292.

9. Gaffoor, I., D. W. Brown, R. Plattner, R. H. Proctor, W. H. Qi, and F. Trail. 2005. Functional analysis of the polyketide synthase genes in the filamentous fungus Gibberella zeae (Anamorph Fusarium graminearum). Eukaryotic Cell 4: 1926-1933.

10. Goswami, R. S. and H. C. Kistler. 2004. Heading for disaster: Fusarium graminearum on cereal crops. Mol. Plant Pathol. 5:515-525.

11. Harris, L. J., A. E. Desjardins, R. D. Plattner, P. Nicholson, G. Butler, J. C. Young, G. Weston, R. H. Proctor, and T. M. Hohn. 1999. Possible role of trichothecene mycotoxins in virulence of Fusarium graminearum on maize. Plant Disease 83:954-960.

12. Igawa, T., N. Takahashi-Ando, N. Ochiai, S. Ohsato, T. Shimizu, T. Kudo, I. Yamaguchi, and M. Kimura. 2007. Reduced contamination by the Fusarium mycotoxin zearalenone in maize kernels through genetic modification with a detoxification gene. Applied and Environmental Microbiology 73:1622-1629.

13. J ensen, B., I. M. B. Knudsen, and D. F. Jensen. 2000. Biological seed treatment of cereals with fresh and long-term stored formulations of Clonostachys rosea: Biocontrol efficacy against Fusarium culmorum. European J ournal of Plant Pathology 106:233-242. 
14. Kakeya, H., N. Takahashi-Ando, M. Kimura, R. Onose, I. Yamaguchi, and H. Osada. 2002. Biotransformation of the mycotoxin, zearalenone, to a nonestrogenic compound by a fungal strain of Clonostachys sp. Biosci. Biotechnol. Biochem. 66:2723-2726.

15. Kim, Y. T., Y. R. Lee, J. M. Jin, K. H. Han, H. Kim, J. C. Kim, T. Lee, S. H. Yun, and Y. W. Lee. 2005. Two different polyketide synthase genes are required for synthesis of zearalenone in Gibberella zeae. Molecular Microbiology 58:1102-1113.

16. Knudsen, I. M. B. 1994. Biological control of seed borne diseases. PhD thesis. $\mathrm{PhD}$ thesis. Frederiksberg: The Royal Veterinary and Agricultural University.

17. Knudsen, I. M. B., J. Hockenhull, and D. F. J ensen. 1995. Biocontrol of seedling diseases of barley and wheat caused by Fusarium culmorum and Bipolaris sorokiniana - Effects of selected fungal antagonists on growth and yield components. Plant Pathology 44: 467-477.

18. Logrieco, A., G. Mule, A. Moretti, and A. Bottalico. 2002. Toxigenic Fusarium species and mycotoxins associated with maize ear rot in Europe. European J ournal of Plant Pathology 108:597-609.

19. Maier, F. J., T. Miedaner, B. Hadeler, A. Felk, S. Salomon, M. Lemmens, H. Kassner, and W. Schafer. 2006. Involvement of trichothecenes in fusarioses of wheat, barley and maize evaluated by gene disruption of the trichodiene synthase (Tri5) gene in three field isolates of different chemotype and virulence. Mol. Plant Pathol. 7:449-461.

20. Martins, M. L. and H. M. Martins. 2002. Influence of water activity, temperature and incubation time on the simultaneous production of deoxynivalenol and zearalenone in corn (Zea mays) by Fusarium graminearum. Food Chemistry 79:315-318.

21. Munkvold, G. P. 2003. Epidemiology of Fusarium diseases and their mycotoxins in maize ears. European J ournal of Plant Pathology 109:705-713.

22. Parry, D. W., P. J enkinson, and L. Mcleod. 1995. Fusarium ear blight (scab) in small grain cereals - A review. Plant Pathology 44:207-238.

23. Reid, L. M., D. E. Mather, and R. I. Hamilton. 1996. Distribution of deoxynivalenol in Fusarium graminearum infected maize ears. Phytopathology 86:110-114.

24. Shimada, T. and M. Otani. 1990. Effects of Fusarium mycotoxins on the growth of shoots and roots at germination in some J apanese wheat cultivars. Cereal Research Communications 18:229-232.

25. Sutton, J. C. 1982. Epidemiology of wheat head blight and maize ear rot caused by Fusarium graminearum. Canadian Journal of Plant Pathology 4: 195.

26. Takahashi-Ando, N., M. Kimura, H. Kakeya, H. Osada, and I. Yamaguchi. 2002. A novel lactonohydrolase responsible for the detoxification of zearalenone: enzyme purification and gene cloning. Biochem.J . 365:1-6. 
27. Utermark, J. and P. Karlovsky. 2007. Role of zearalenone lactonase in protection of Gliocladium roseum from fungitoxic effects of the mycotoxin zearalenone. Applied and Environmental Microbiology 73:637-642.

28. Vakili, N. G. 1992. Biological seed treatment of corn with mycopathogenic fungi. Journal of Phytopathology-Phytopathologische Zeitschrift 134:313323.

29. Volkl, A., G. Woerfel, T. Haeussermann, B. Vogler, I. Klaiber, and P. Karlovsky. 1997. Biotransformation von Mykotoxinen von Fusarium spp. Proceedings of 19th Mykotoxin-Workshop, Munich, Germany, 2-4J uni 1997.

30. Wang, H., S. F. Hwang, F. Eudes, K. F. Chang, R. J. Howard, and G. D. Turnbull. 2006. Trichothecenes and aggressiveness of Fusarium graminearum causing seedling blight and root rot in cereals. Plant Pathology 55:224-230.

31. Xue, A. G. 2003. Biological control of pathogens causing root rot complex in field pea using Clonostachys rosea strain ACM941. Phytopathology 93:329335.

32. Yoshida, M. and T. Nakajima. 2010. Deoxynivalenol and nivalenol accumulation in wheat infected with Fusarium graminearum during grain development. Phytopathology 100:763-773.

33. Zhang, Y. J., P. S. Fan, X. Zhang, C. J. Chen, and M. G. Zhou. 2009. Quantification of Fusarium graminearum in harvested grain by real-time polymerase chain reaction to assess efficacies of fungicides on Fusarium head blight, deoxynivalenol contamination, and yield of winter wheat. Phytopathology 99:95-100. 


\section{Summary}

F. graminearum is an important pathogen that causes head blight and root rot in wheat and ear and root rot in maize. Because of its toxigenic nature most of the diseased crops are found to accumulate large pool of mycotoxins. Trichothecenes type B deoxynivalenol (DON) its acetylated derivatives, nivalenol (NIV) and estrogenic zearalenone (ZEN) are major mycotoxins produced by the fungus. The DON occurrence is high in cereals while NIV and ZEN are potential contaminants in maize. The biological role of these mycotoxins is not fully elucidated yet.

We investigated the mixed colony incubation and ecological role of ZEN in rhizosphere colonization of maize and wheat root infection. The G. roseum which detoxifies ZEN and its knock-out non-detoxifying mutants were generated and tested earlier in our research group. We found out that mixed incubation of colonies of G. roseum wild-type and Zes2 mutant started to grow the same time on pink-covered surface of $F$. graminearum on YES medium. The wild-type colony size however increased multiple-fold and appeared as hollowed spots or digested pits on pathogen surface and pigmentation changed at borders from light-pale to darkorange over 3-5 days incubation. In contrast, Zes2 mutant colony diameter stayed compact as no large expansion was measured. In rhizosphere colonization trials 5 aggressive $F$. graminearum strains co-inoculated with G. roseum wild-type and Zes2 mutant on maize and wheat root. Significant differences were observed between G. roseum wild-type and Zes2 mutant co-inoculated with F. graminearum. The coinoculated seedlings with $G$. roseum wild-type and pathogen showed increased emergence, larger yields in root and shoot indexes and lesser disease symptoms while Zes2 mutant resulted in partial protection in seedlings of maize and wheat. We may conclude that ZEN is involved in substrate colonization and inhibition would leave its producer in hostile ecological environments to protect the resource from its competitors.

Twenty-two F. graminearum strains were investigated in wheat-maizepathosystem. The greater disease severity was observed on wheat heads; however increased root infection took place in maize. The strains virulence was positively correlated among wheat heads/ maize ears and root infections of wheat and maize. The DON contents were also positively correlated with disease severity in both wheat and maize ears. However, no positive correlation was found between DON 
contents and fungal DNA in maize because of different chemotypes. In wheat, tight correlation was proved between DON and DNA contents. NIV was detected in lower amount than DON while all maize ears were quantified for ZEN accumulation. In our study some of the strains were also found to produce 15ADON on maize which is of great concern. In both wheat and maize ears the amount of mycotoxins was a decisive factor in pathogen virulence. We may conclude that wheat heads are susceptible than root while opposite is true in maize that both ears and roots are observed susceptible to Fusarium infection.

We investigated the role of DON and NIV in wheat and maize root infection. We inoculated the seeds of both plants with two F. graminearum wild-type strains specific to DON and NIV chemotypes and two individual deficient mutants correspond to each chemotypes. Wheat inoculated with NIV wild-type showed poor emergence, reduced root and shoot indexes and increased disease severity compared to the deficient mutants. The DNA contents showed larger amount in NIV wild-type infected wheat root or shoot than traces of DNA detected in deficient mutants. NIV was detected in traceable amounts but with great variability among samples of wheat or below the detection limits. In maize, either NIV wild-type or deficient mutants both produced same magnitude of disease. DON wild-type inoculated wheat suffered extreme losses in germination, resulted in reduced biomass indexes and greater disease severity than deficient mutants. No significant differences observed between DON wild-type and deficient mutants on maize root. We conclude that NIV and DON are virulent factor on wheat not on maize root infection. 


\section{Acknowledgements}

My best complements and sincere gratitude to Prof. Petr Karlovsky, who provided me such a great opportunity to conduct my Doctoral research under his supervision. I would never forget his wonderful mentorship, discussions and positive critics during my work and financial support to research work.

I like to thank Prof. Elke Pawelzik, who kindly accepted to serve as co-supervisor for this Doctoral research.

I am very thankful to Dr. Bettina Kessel, KWS Saat AG Einbeck, Prof. Jan Schirawski, University of Goettingen and Federal Government Institute Darmstadt, Germany for providing the maize and wheat seeds and Prof. W. Schaefer and Dr. Frank Maier, University of Hamburg for providing us the Fusarium graminearum strains.

I also gratefully acknowledge Sabine Nutz for her technical assistance in maize and wheat trials and to proofread the thesis with Ruth. Teresa Galvan help to conduct several greenhouse and lab experiments.

I like to pass my special thanks to Heike and Patricia who helped me solve many technical problems. I also acknowledge the technical help of Katja, Jacqueline and technical assistance from Marcel and Mona.

My whole-hearted regards to Dr. Arne Weiberg, Dr. Ramesh Subramani, Dr. Richard Splivallo and Dr. Subhankar Chatterjee for their guidance, scientific discussions and great company.

I like to thank all colleagues in Molecular Phytopathology and Mycotoxin Group and General Plant Pathology Group for such great atmosphere.

I like to thank Mr. Hodyl for taking care of several experiments in Greenhouse.

I would also never forget Dr. A.R. Asif, Department of Clinical Chemistry, University of Goettingen, for his discussions, critics and guidelines during my work and all the way great emotional support during my stay.

I deeply feel the love and support of my eldest brothers Khalique and Amir throughout my studies and life. My sweet lovely sisters Amina and Samina who travelled with me all the way in my imaginations, in dreams, in hardships after parents, who have been there for me forever and always, sacrificed their interest for my benefits. 


\section{Scientific Contributions}

The role of zearalenone detoxification in the protection of maize against $F$. graminearum. Poster Presentation at 31st Mycotoxin Workshop 15-17 J une 2009 Muenster, Germany

The role of deoxynivalenol (DON) and nivalenol (NIV) in the infection of roots of wheat and maize with F. graminearum. Lecture at 32nd Mycotoxin Workshop 14-16 J une 2010 Lyngby, Denmark

\section{Author's Contributions}

Awais Ahmed (AA), Stephan Bucher (SB), Katharina Doell (KD) and Petr Karlovsky (PK).

\section{Manuscript 01.}

Role of zearalenone in the interaction of $F$. graminearum with $G$. roseum in vitro and in wheat and maize rhizosphere.

(AA) carried out the experiments, designed the spreadsheets and drafted parts of the manuscript. (PK) conceived and guided the study, and wrote parts of the manuscript.

\section{Manuscript 02.}

Pathogenicity and mycotoxins content of different F.graminearum strains on maize and wheat ear and root infection.

(AA) carried out the experiments, designed the spreadsheets and drafted parts of the manuscript. (KD) performed all mycotoxin analysis. (PK) conceived and guided the study, and wrote parts of the manuscript.

\section{Manuscript 03.}

Role of deoxynivalenol (DON) and nivalenol (NIV) in wheat and maize root infection by $F$. graminearum.

(AA) carried out the experiments, designed the spreadsheets and drafted parts of the manuscript. (SB) carried out part of experiments and fungal DNA quantification. (KD) performed all mycotoxin analysis. (PK) conceived and guided the study, and wrote parts of the manuscript. 


\section{Curriculum Vitae}

\section{Personal details}

$\begin{array}{ll}\text { Name: } & \text { Awais Ahmed } \\ \text { Sex: } & \text { Male } \\ \text { Date of birth: } & \text { 30-08-1978 } \\ \text { Nationality: } & \text { Pakistan }\end{array}$

Doctoral Studies

2007-2010 Doctoral Studies in Agricultural Sciences (IPAG) - Molecular Phytopathology and Mycotoxin Research Unit, University of Goettingen, Germany

Thesis title: The biological role of Fusarium graminearum mycotoxins

\section{Graduate Studies}

2005-2007 Masters of Science in Agriculture and Resource Management in the Tropics and Subtropics, University of Bonn, Germany Thesis title: Efficacy of different Trichoderma species against root-knot nematode Meloidogyne incognita on tomato

2003-2004 Master of Science in Sustainable Resource Management, Technical University Munich, Germany. (2-semester studies)

\section{Under Graduate Studies}

1998-2002 Bachelor of Science in Agriculture (Hons) Entomology/ Plant Pathology. Sind Agriculture University Tandojam, Pakistan

1996-1997 Higher Secondary School Certificate Examinations Government College, Nawabshah, Pakistan

\section{Work Experience}

Since 09/2007 Research Assistant at Department of Molecular Phytopathology and Mycotoxin Research Unit, University of Goettingen. 